\title{
Synaptic physiology of the developing Drosophila neuromuscular junction
}

\author{
Dissertation \\ zur Erlangung des Doktorgrades \\ der Mathematisch-Naturwissenschaftlichen Fakultäten \\ der Georg-August-Universität zu Göttingen
}

vorgelegt von

Robert Johannes Kittel

aus Berlin

Göttingen 2006 
D 7

Referent: Prof. Dr. Erwin Neher

Korreferent: Prof. Dr. Friedrich-Wilhelm Schürmann

Tag der mündlichen Prüfung: 01.11.2006 


\section{Acknowledgements}

I would like to thank my instructor Prof. Dr. Stephan Sigrist for giving me the opportunity to conduct these studies in his research group. Over the past years, Stephan has been my major scientific mentor. His excellent supervision and friendship were decisive for this work. I also thank my supervisor Prof. Dr. Erwin Neher and my coreferee Prof. Dr. Friedrich-Wilhelm Schürmann for sharing their experience in discussions and advising me on my project. I would like to express my appreciation to Prof. Dr. Manfred Heckmann for excellent guidance and support in learning electrophysiological techniques and for the friendly atmosphere during collaborations. Thank you also to Dr. Christian Pawlu and to Dr. Stefan Hallermann for sharing their expertise on both scientific and non-scientific issues. For further collaborations, I would like to thank Prof. Dr. Erich Buchner, Prof. Dr. Stefan Hell, and Dr. Anne Ephrussi. I thank Dr. Carolin Wichmann for excellent electron micrographs and work on the Bruchpilot mutant, Wernher Fouquet for beautiful images, Andreas Schmid and Dr. Gang Qin for work on receptors, Sara Mertel and Dr. Florence Besse for work on Basigin, Andreas Frölich for work on GluRIIB, and Dr. Tobias Rasse for work on both receptors and Bruchpilot. I would like to thank Christine Quentin and Franziska Zehe for excellent technical assistance, Wiebke Heinrich for friendly and reliable coordination, and Frank Kötting for sound technical constructions. Dr. Dave Featherstone I thank for preparation techniques and Dr. Jakob Sørensen for the autocorrelation analysis. Thank you also to all present and past ENI members for contributing to the unique working atmosphere. Especially Heiko Röhse, Dr. Emanuele Cocucci, Drs. Laura and Massimiliano Stagi, Dr. Manuela Schmidt, Miriam Richter, Magda Krause, Dr. Simon Sedej, Oliver Schade, Tobias Schwarz, Dr. Carola Sigrist, Dr. Carlos Merino, Dr. Tobias Rose, Rui Tian, David Owald, Dr. Jan Turner, and Dr. Stephan Speier. Thank you to my friends, particularly Niki and Christoph, for tolerating monologues on synaptic function and to Gottfried Ostermeier for insightful interdisciplinary discussions. I would like to thank my parents Margaret and Harald for patience and encouragement and mein Schwesterherzchen Anna for spiritual guidance. Finally, I thank Imke for enduring support, understanding, and a wonderful time. 


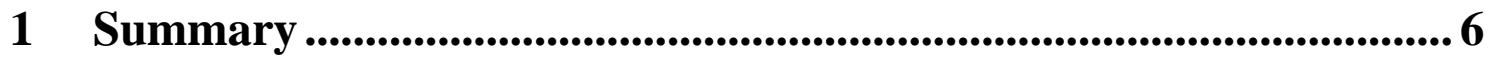

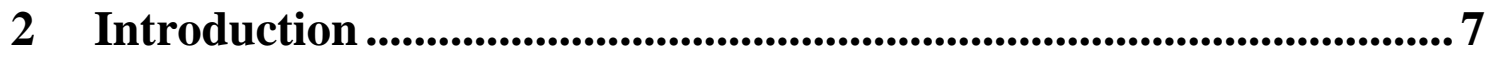

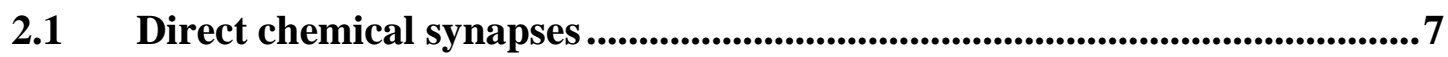

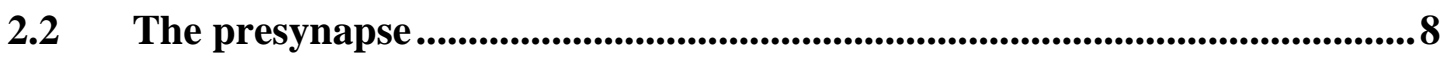

2.2.1 The role of $\mathrm{Ca}^{2+}$ ions in synaptic transmission ................................... 8

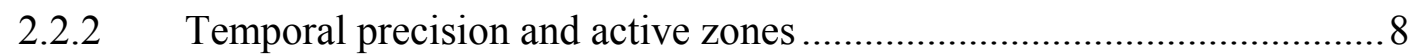

2.2.3 Release probability and vesicle pools ................................................. 11

$2.3 \quad$ The postsynapse..............................................................................................................13

2.3.1 The involvement of AMPA-type glutamate receptors in synaptic

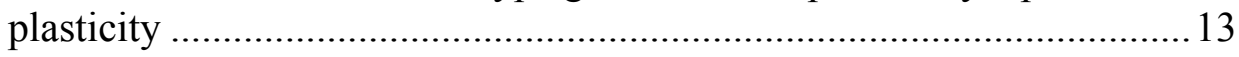

2.3.2 Synaptic micro-environments and PDZ-domain-proteins ..................... 14

2.4 Assembly and maintenance of synaptic domains ..................................16

$2.5 \quad$ Drosophila melanogaster ........................................................................18

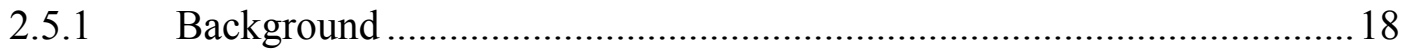

2.5.2 Physiology and anatomy of the neuromuscular junction ........................19

2.5.3 Glutamate receptor subunit expression influences synaptic strength and morphology at the Drosophila NMJ...................................................24

3 Methods ................................................................................................ 28

3.1 Principles of operation........................................................................................28

3.1.1 Voltage clamping with two microelectrodes......................................28

3.1.2 Voltage clamping with a single patch pipette ...................................... 33

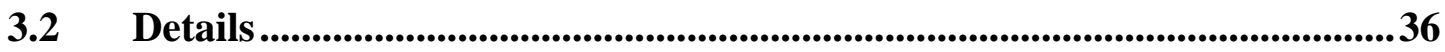

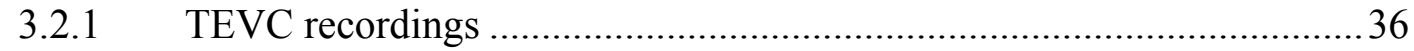

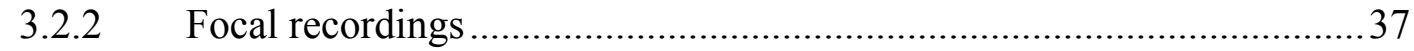

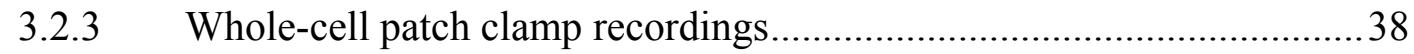

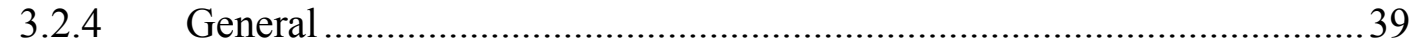

$4 \quad$ Results........................................................................................................... 40

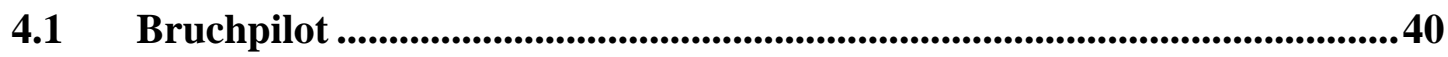


4.1.1 The monoclonal antibody Nc82 recognises Bruchpilot .........................40

4.1.2 Synaptic function following suppression of Bruchpilot......................... 43

4.1.3 Synaptic function following complete elimination of Bruchpilot............46

\subsection{Drosophila glutamate receptor subunits GluRIIA and GluRIIB ............55}

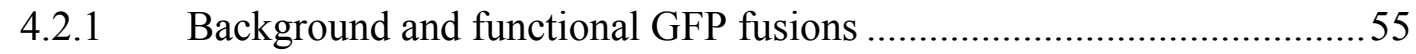

4.2.2 Differential influences on postsynaptic sensitivity ...............................56

4.2.3 Effects of minimal glutamate receptor expression...............................59

4.3 Drosophila Basigin..............................................................................62

4.3.1 Maintenance of synaptic domains by perisynaptic Basigin ...................62

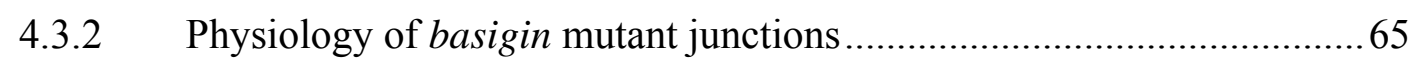

4.3.3 Association of Basigin with the actin network .....................................6 68

5 Discussion ......................................................................................... 71

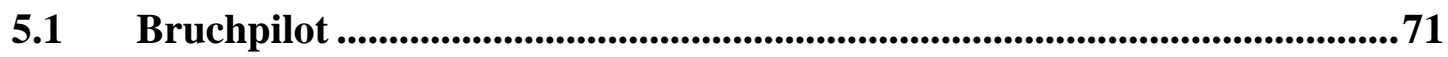

5.1.1 Bruchpilot and $\mathrm{Ca}^{2+}$ channels............................................................. 71

5.1.2 Localisation of Bruchpilot within the active zone ............................... 74

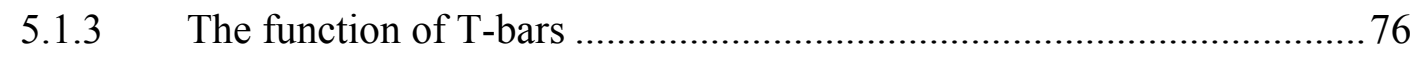

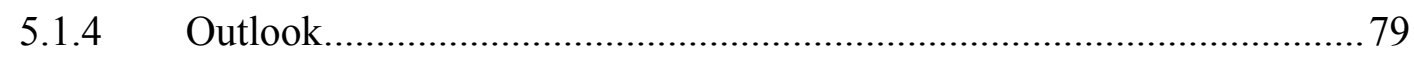

\subsection{Drosophila glutamate receptor subunits GluRIIA and GluRIIB .............80}

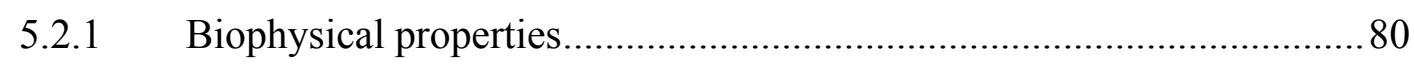

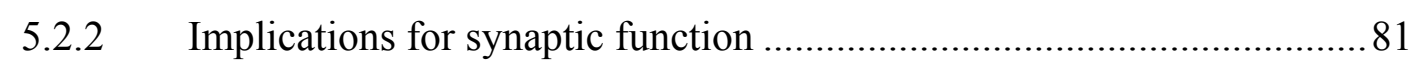

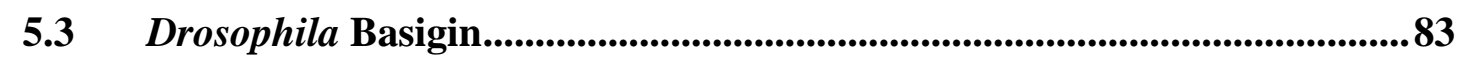

5.3.1 Involvement of Basigin in vesicle mobilisation.................................. 83

5.3.2 Association of Basigin with the actin cytoskeleton .................................85

6 Abbreviations.................................................................................. 87

7 Table of Figures............................................................................... 89

8 References ..................................................................................... 91

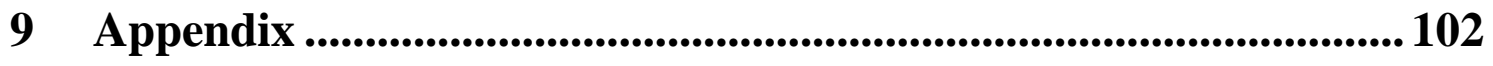

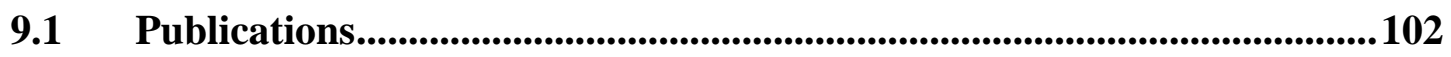

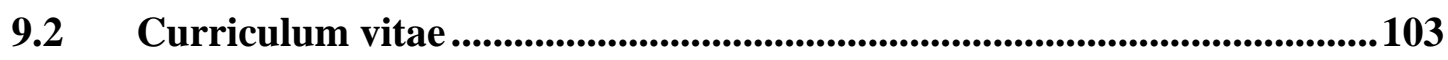




\section{Summary}

There is a large increase in the number of synapses, and a constant structural and functional remodelling of already established synaptic sites, during the development of both the vertebrate and invertebrate nervous systems. Particular forms of such synaptic modifications are regarded as essential for learning processes, and the stabilisation of such modulations is believed to mediate the consolidation of acquired information, in other words - memory. In this context, it remains unclear how a neuron translates a developmental or activity-dependent signal into a moderate but physiologically relevant alteration in functional or structural synaptic connectivity.

Throughout the development of the Drosophila melanogaster neuromuscular junction (NMJ), structural and functional remodelling of glutamatergic synaptic connections takes place at high rates. The work described in this thesis exploits this feature, by examining situations where proteins implicated in synaptic function are interfered with. More specifically, electrophysiological analyses were performed to assess how the synaptic physiology responds to such manipulations. Thereby, the role of candidate proteins in synaptic performance could be deduced, adding to our understanding of the system.

The results described were obtained in several case studies of proteins involved in pre-, post-, and perisynaptic function. (1) The novel presynaptic protein Bruchpilot is essential for both the structural and functional integrity of active zones. By establishing proximity between calcium channels and vesicles Bruchpilot promotes efficient neurotransmitter release and enables patterned synaptic plasticity. (2) The functional properties and differential effects of the postsynaptic ionotropic glutamate receptor subunits GluRIIA and IIB on synaptic efficacy are described. And finally, (3) the novel perisynaptic immunoglobulin domain-containing protein Basigin is introduced. Basigin contributes to the integrity of the presynaptic actin cytoskeleton, and to the precision of synchronised neurotransmitter release. The results not only illustrate a role of these proteins in basic synaptic performance, but also imply the recruitment of their functional pathways, required for mediating processes of structural and functional synaptic development and plasticity. 


\section{Introduction}

\subsection{Direct chemical synapses}

Charles Scott Sherrington first coined the term 'synapse' as a physiological concept in 1897. Derived from Greek, meaning to join together (syn- 'together' + hapsis 'joining'), synapses represent intercellular contact sites, at which neurons communicate with their partner cells. At direct, or 'fast', synapses, the rapid transmission of information is mediated by the flow of electrical current from the pre- to the postsynaptic cell. The current may flow directly from one cell to another (electrical synapse), or rely on an intermediate step, involving the release and detection of chemical transmitter substances (chemical synapse). Importantly, the mechanism of chemical synaptic communication enables the strength of signal transmission to be adapted in a highly variable manner (Magleby, 1987), and thereby synapses filter, integrate or modify information, thus acting as key regulators of many complex brain processes. Particularly, the activitydependent modulation of synaptic strength, termed 'synaptic plasticity' (Konorski, 1948), has received much attention in recent decades. The concept, that learning involves plastic changes of synaptic connections and that memory storage requires the stabilisation of such modulations was already proposed in 1894 by Santiago Ramón y Cajal (Ramón y Cajal, 1894) and later refined by Donald Olding Hebb (Hebb, 1949). Systematic investigations of both the invertebrate nervous system (Kandel, 1976, 2001) and the mammalian hippocampus (Bliss and Lømo, 1973; Bliss and Collingridge, 1993) support the hypothesis that the plasticity of chemical synapses is fundamental for certain forms of learning.

Direct chemical synaptic communication is mediated by the depolarisation-induced fusion of neurotransmitter-filled vesicles with the presynaptic membrane and the subsequent activation of specific postsynaptic receptor ion channels, permitting current flow and conveying signal transduction. Before reviewing the postsynaptic reception apparatus, the role of calcium in vesicle fusion and the specialised site of transmitter release, the presynaptic active zone, are introduced. 


\subsection{The presynapse}

\subsubsection{The role of $\mathrm{Ca}^{2+}$ ions in synaptic transmission}

The divalent cation calcium $\left(\mathrm{Ca}^{2+}\right)$ was found necessary for the successful transmission of nerve impulses, more than a century ago (Locke, 1894), even before the concept of chemical synaptic transmission was established (Loewi, 1921). Further work (Feng, 1940; Kuffler, 1942; Del Castillo and Stark, 1952) led to the calcium hypothesis which, combined with the quantal release hypothesis (Del Castillo and Katz, 1954), proposed that the release, or exocytosis, of neurotransmitter from synaptic vesicles is triggered by elevations of the $\mathrm{Ca}^{2+}$ concentration in the presynaptic terminal (Katz and Miledi, 1965). It was realised that the invasion of the terminal by an action potential (AP) triggers the opening of voltage-gated $\mathrm{Ca}^{2+}$ channels and that the exact amplitude and time course of the ensuing $\mathrm{Ca}^{2+}$ influx dictate the amplitude and time course of release (Barrett and Stevens, 1972). Instead of being distributed evenly, presynaptic $\mathrm{Ca}^{2+}$ signals describe highly localised (tens of nanometers), transient (tens of microseconds) microdomains in the vicinity of $\mathrm{Ca}^{2+}$ channels (Llinas et al., 1981; Chad and Eckert, 1984; Augustine and Neher, 1992). Both the distance between $\mathrm{Ca}^{2+}$ channels and the distance between $\mathrm{Ca}^{2+}$ channels and vesicles at release sites therefore influence the transmission characteristics of a synapse (Neher, 1998).

\subsubsection{Temporal precision and active zones}

Synaptic communication is very rapid, and correspondingly synapses display several important features that enable the presynaptic influx of $\mathrm{Ca}^{2+}$ to be followed by a postsynaptic response on the sub-millisecond time scale. Both the tight alignment of pre- and postsynaptic membranes and the specialised presynaptic region of exocytosis, the active zone (Couteaux and Pecot-Dechavassine, 1970; Landis et al., 1988), reflect the requirement for rapid signal transduction. On the ultrastructural level, active zones are distinct morphological specialisations consisting of an electron-dense membrane and associated cytomatrix, frequently identified by projections reaching into the cytoplasm, which are surrounded by clusters of neurotransmitter-filled synaptic vesicles (Fig. 1AC). The extent of these electron-dense projections varies greatly between synapse types, 
ranging from roughly $50 \mathrm{~nm}$ high pyramidally shaped particles in synapses of the mammalian central nervous system [CNS; (Phillips et al., 2001)], over approximately $70 \mathrm{~nm}$ long T-shaped protrusions (T-bars) at the Drosophila NMJ (Atwood et al., 1993), to the spherical synaptic ribbons found in vertebrate sensory synapses which extend 0.5 $1 \mu \mathrm{m}$ into the cytoplasm (Lenzi and von Gersdorff, 2001). These structural differences most likely reflect the physiological demands set by the synaptic contact (Zhai and Bellen, 2004). While the function of such projections is still under intense debate, a popular model suggests that ribbons tether synaptic vesicles and act as a conveyer belt to deliver vesicles to sites of exocytosis, thereby sustaining the graded continuous neurotransmitter release of sensory synapses (von Gersdorff, 2001).

As one of the fastest cell biological processes, $\mathrm{Ca}^{2+}$-triggered neurotransmitter release requires a molecular coupling of $\mathrm{Ca}^{2+}$ influx with vesicle fusion at the protein level (Rosenmund et al., 2003). The fusion of vesicles with the active zone membrane presumably follows binding of $\mathrm{Ca}^{2+}$ to the vesicle protein synaptotagmin (Geppert et al., 1994; Koh and Bellen, 2003), and is mediated by SNARE [soluble $N$-ethylmaleimidesensitive factor attachment protein (SNAP) receptor] proteins, that include Synaptobrevin on synaptic vesicles and SNAP-25 and Syntaxin on the plasma membrane (Jahn et al., 2003; Südhof, 2004).

To ensure rapid and efficient stimulus-secretion coupling, active zones display clusters of voltage-gated $\mathrm{Ca}^{2+}$ channels close to vesicle docking sites. An elegant study of the frog neuromuscular junction used electron tomography to reconstruct the three dimensional structure of the cytomatrix at the active zone [CAZ; (Harlow et al., 2001)]. The results show two rows of docked synaptic vesicles with a central electron-dense 'beam' in between. From this beam, 'ribs' extend to contact, and possibly anchor, both the vesicles and intra-membrane particles, termed 'pegs', which are hypothesised to represent $\mathrm{Ca}^{2+}$ and $\mathrm{Ca}^{2+}$-activated $\mathrm{K}^{+}\left(\mathrm{K}_{\mathrm{Ca}}\right)$ channels (Fig. 1D). 

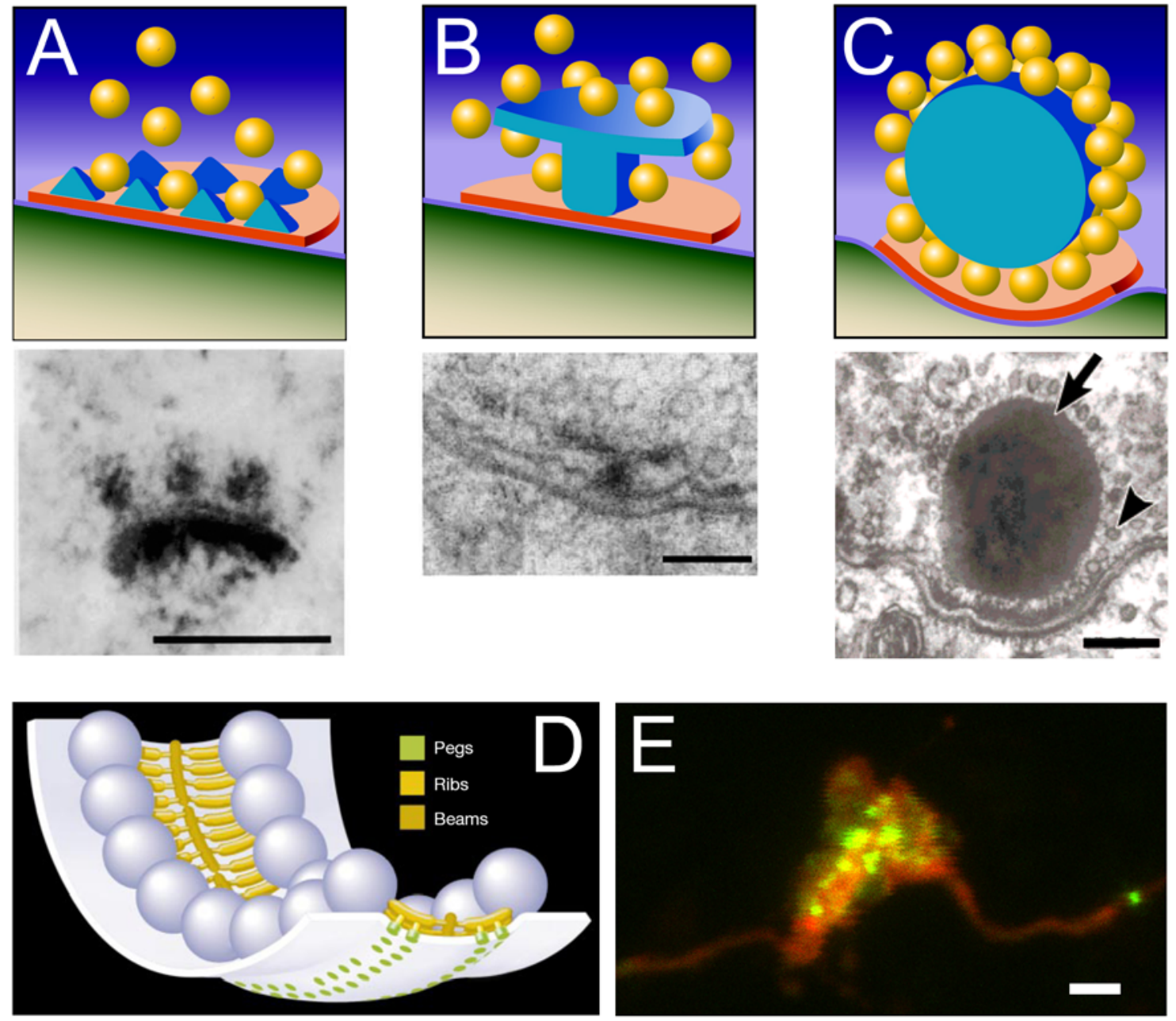

Figure 1. Ultrastructure of the active zone.

(A-C) Schematic representations (Zhai and Bellen, 2004) and electron micrographs of (A) dense bodies at a mammalian CNS synapse (Phillips et al., 2001), (B) a T-bar at the Drosophila NMJ (Atwood et al., 1993), and (C) a synaptic ribbon (arrow) of the frog inner ear hair cell surrounded by a halo of synaptic vesicles [arrowhead; (Lenzi and von Gersdorff, 2001)]. (D) Schematised arrangement of pegs, ribs, beams and vesicles at the frog NMJ (Harlow et al., 2001). (E) Confocal image of a mossy fibre bouton from a transiently transfected mouse hippocampal granule cell. CAST1-EGFP (green) accumulates in clusters, which probably identify individual active zones. The cytoplasm is marked with DsRed cDNA [red; (Kittel et al., 2006a)]. Scale bars, $200 \mathrm{~nm}$ in (A, C), $100 \mathrm{~nm}$ in (B), and $2 \mu \mathrm{m}$ in (E).

The spatial and functional arrangement of $\mathrm{Ca}^{2+}$ channels within active zones appears to be organised through interactions with active zone proteins which may define 'slots'loci of interaction that ultimately govern synaptic efficacy (Harlow et al., 2001; Cao et al., 2004). However, the identification of these active zone proteins has lagged behind the characterisation of ion channels, synaptic vesicle proteins, and the fusion machinery, and fundamental components still await a functional characterisation (Garner et al., 
2000b). One such candidate is CAST (cytomatrix of the active zone-associated structural protein). CAST is enriched in active zones, it interacts with prominent CAZ proteins (Ohtsuka et al., 2002; Takao-Rikitsu et al., 2004), and it may serve as an active zone label [Fig. 1E; (Hagiwara et al., 2005)]. The interaction partners include Bassoon, which is required for the structural integrity of active zones (tom Dieck et al., 1998; Khimich et al., 2005), Piccolo (Fenster et al., 2000), Munc 13-1 [mammalian homologue of the nematode C. elegans Unc13 (uncoordinated) protein], an essential factor for the priming process of vesicles in the CNS (Augustin et al., 1999), and Rim1 (Rab3-interacting molecule-1) which provides a direct link between synaptic vesicles and the active zone (Wang et al., 2000; Betz et al., 2001).

\subsubsection{Release probability and vesicle pools}

Within the presynaptic terminal, vesicles participate in a cycle of exocytosis at the active zone and endocytosis at the adjacent periactive zone, thereby enabling rapid and repeated use (Südhof, 2004). Of these vesicles, only a small fraction is docked to the synaptic membrane, while the rest reside in adjacent clusters. A number of attempts have been made to assign vesicles to distinct 'pools', reflecting particular functional properties. A popular model suggests the distinction of three pools (Zucker and Regehr, 2002; Rizzoli and Betz, 2005): the readily releasable pool, consisting of vesicles docked to the active zone membrane and primed for release; the recycling pool of vesicles which maintain transmitter release during moderate physiological stimulation; and the reserve pool, defined as a storage depot of synaptic vesicles which participate in release only during strong stimulation and after the recycling pool has been exhausted.

The number of vesicles released at a synapse is determined by the number of releaseready vesicles and the exocytotic probability of the individual vesicles. Low release probability synapses often display facilitation and augmentation whereas high release probability synapses tend to exhibit paired-pulse and frequency-dependent depression (Zucker and Regehr, 2002). Release probability and the response to repeated use can differ, however, even for synapses of a single neuron, in a target dependent manner. For example, high release probability synapses of pyramidal neurons in layer $2 / 3$ of the rat neocortex on multipolar interneurons have higher presynaptic $\mathrm{Ca}^{2+}$ signals than the low 
release probability synapses on bitufted interneurons (Rozov et al., 2001; Koester and Johnston, 2005). The precise organisation of the presynaptic active zone including the density, coupling and direct juxtaposition of $\mathrm{Ca}^{2+}$ channels and synaptic vesicles are considered critical in this context (Atwood and Karunanithi, 2002).

The identification of sub-populations of rapidly and reluctantly releasable vesicles within the pool of readily releasable vesicles (Sakaba and Neher, 2001; Schneggenburger and Neher, 2005), illustrates limitations of the simple three pool model. One interpretation of this observation is that differences in the distance between $\mathrm{Ca}^{2+}$ channels and vesicles lead to heterogeneous fusion kinetics upon $\mathrm{Ca}^{2+}$ influx (Neher, 1998). 


\subsection{The postsynapse}

\subsubsection{The involvement of AMPA-type glutamate receptors in synaptic plasticity}

Glutamate is the major excitatory neurotransmitter in the mammalian central nervous system (Seeburg, 1993). At glutamatergic synapses, fast excitatory transmission acts through binding of glutamate to receptors located in the postsynaptic density (PSD) and the subsequent opening of receptor-coupled ion channels to permit cation influx and postsynaptic depolarisation. In vertebrates, these ionotropic receptors are divided into three broad classes named after dicarboxylic amino acids that are selective agonists: NMDA ( $N$-methyl-D-aspartate), kainate, and AMPA [ $\alpha$-amino-3-hydroxyl-5-methyl-4isoxalone propionic acid; (Dingledine, 1999)]. NMDA receptors have several unusual permeability properties, including high permeability to $\mathrm{Ca}^{2+}$ ions, long channel burst time, and voltage dependence (Hille, 2001). These receptors are widely regarded as the main triggers for the induction of long-term potentiation (LTP), a form of synaptic plasticity that, together with long-term depression (LTD), has become a popular model for studying the molecular basis of learning (Collingridge et al., 2004). Kainate receptors can trigger NMDA-receptor-independent forms of LTP and are thought to be key modulators of network activity (Pinheiro and Mulle, 2006). AMPA receptors, in turn, express the synaptic response during LTP and are regarded as important targets of modulation during synaptic plasticity (Bliss and Collingridge, 1993; Linden and Connor, 1995; Collingridge et al., 2004).

In brief, AMPA receptors are likely hetero-oligomeric complexes (Rosenmund et al., 1998; Madden, 2002). The receptor subunits GluR1-GluR4 are expressed in the mouse hippocampus (Seeburg, 1993; Dingledine et al., 1999). Importantly, this system has been used to show that the AMPA receptor subunit composition determines the function of these receptor complexes throughout synaptic plasticity. Thus during LTP, GluR1/GluR2 receptors appear to be transported to the synapse (Shi et al., 2001), thereby converting previously silent sites into active synapses. This process is activitydependent and leads to an enhancement of transmission. GluR2/GluR3 receptor complexes, in contrast, continuously replace already synaptically localised GluR1/GluR2 receptors, and are thereby thought to preserve the plastic changes (Zhu et al., 2000). 
Receptors may either be recruited into PSDs from a diffuse plasma membrane pool by lateral migration (Borgdorff and Choquet, 2002), or from intracellular compartments via subunit-specific pathways, possibly using preformed slots established at the postsynaptic membrane (Barry and Ziff, 2002). The rapid modulation of the number of synaptically expressed receptors might also be mediated by cytoplasmic receptorbearing vesicles together with exo- and endocytotic mechanisms (Malinow and Malenka, 2002). Furthermore, the subunit-specific regulation of transport and synaptic presentation of receptor complexes appears to involve interactions with so-called PDZdomain-proteins (Shi et al., 2001).

\subsubsection{Synaptic micro-environments and PDZ-domain-proteins}

In order for a metazoic cell to communicate effectively with its environment, the relevant receptor and effector proteins must first be transported to their specific designated region, and then organised at their destination. In neurons this 'polarisation' is particularly apparent, and is reflected by their highly specialised axonal and dendritic compartments. The membrane polarisation is organised by temporally and spatially defined 'membrane domains', which are specified in terms of lipid and protein composition. In this context, synapses can be looked upon as especially stable and specified membrane domains.

Many proteins involved in the establishment of cellular polarity have a common protein-protein interaction motif called the PDZ-domain, which is conserved throughout the metazoan taxonomic group. The sequence of the PDZ-domain consists of approximately 90 amino-acids, and was originally identified in, and named after, the synaptic scaffolding protein PSD-95/SAP90, its Drosophila orthologue (Discs-large, Dlg), and the epithelial protein ZO-1 (Garner and Kindler, 1996; Craven and Bredt, 1998; Hata et al., 1998). The PDZ-domain binds to the C-terminus of specific transmembrane proteins. The binding specificity of different PDZ-domains is governed by variable amino-acid sequences which line the peptide-binding groove of the PDZdomain (Sheng and Sala, 2001). Within the family of PDZ-domain-proteins, multiple copies of PDZ-domains are frequently found within one protein. In terms of functionality, PDZ-domains appear to organise supra-molecular complexes which, in 
turn, are necessary for local signalling processes, the establishment and maintenance of cell polarity, and the transport of specific proteins. These features hint at an involvement of PDZ-domain-proteins in synaptic plasticity. Indeed, a class of PDZdomain-containing proteins, including GRIP (glutamate receptor interacting protein), ABP (AMPA receptor-binding protein), PICK1 (protein interacting with $\mathrm{C}$ kinase 1) and SAP-97 (synapse-associated protein) have been implicated in synaptic targeting of AMPA receptors (Sheng and Pak, 1999; Braithwaite et al., 2000; Garner et al., 2000a). Recent studies have also demonstrated that a family of small transmembrane AMPA receptor regulatory proteins (TARPs) can control both AMPA receptor trafficking and gating (Nicoll et al., 2006), and that the synaptic targeting of AMPA receptors by the TARP member stargazing requires binding of the C-terminus to the PDZ-domain of PSD-95 (Chen et al., 2000). 


\subsection{Assembly and maintenance of synaptic domains}

Synapses are confronted with a major challenge to maintain their highly organised structure while constantly adapting their morphology and strength in response to developmental programs and external stimuli. Synaptic contacts are able to adjust the size of their terminals, the number and composition of their pre- and postsynaptic membrane specialisations, as well as the availability of release-competent synaptic vesicles. During these dynamic alterations, the system must preserve both the precise physical and functional connections between the pre- and postsynaptic compartments, as well as between cytoplasmic domains and membrane specialisations.

To date, the mechanisms underlying such rearrangements are still poorly understood. Nonetheless, several components of periactive zones, including transmembrane proteins and adaptor molecules, have been implicated in the control of terminal outgrowth, particularly at the Drosophila NMJ (Schuster et al., 1996b; Beumer et al., 1999; Sone et al., 2000; Beumer et al., 2002; Koh et al., 2004; Marie et al., 2004). Cell adhesion molecules of the immunoglobulin superfamily (Ig CAMs) appear involved in maintaining the integrity of synaptic terminals, and transmitting signals to the cell interior. Thereby, they promote the differentiation of pre- and postsynaptic specialisations, and regulate both synaptic structure and function (Crossin and Krushel, 2000; Rougon and Hobert, 2003; Yamagata et al., 2003). At mammalian neuromuscular junctions, the prototypical Ig CAM NCAM (neuronal cell adhesion molecule) is required for the process of synaptic maturation, and more specifically the adequate clustering of $\mathrm{Ca}^{2+}$ channels and SNARE proteins in the presynaptic terminal (PoloParada et al., 2001). At the Drosophila NMJ, the NCAM homologue Fasciclin II (Fas II) is localised to periactive zones and controls both the outgrowth of the NMJ (Schuster et al., 1996b; Stewart et al., 1996; Sigrist et al., 2002), and the establishment and maintenance of synaptic compartments (Sone et al., 2000).

The actin-rich presynaptic cytoskeleton is a further key regulator of synaptic domain rearrangements. In addition, the actin network appears to control the distribution and consequently the availability of synaptic vesicles for release. A number of studies have revealed the presence of a dense network of actin filaments surrounding synaptic vesicles (Shupliakov et al., 2002; Sankaranarayanan et al., 2003; Richards et al., 2004); 
and pharmacological treatments have suggested that this network may modulate the organisation and mobilisation of functionally distinct vesicle pools (Kuromi and Kidokoro, 1998; Sakaba and Neher, 2003). It would thus appear that the actin network links cytoplasmic vesicle pools to specific membrane domains specialised in the exo- or endocytosis of synaptic vesicles. The mechanisms by which plasma membrane and cytoplasmic sub-domains are spatially and functionally connected remains largely unknown. 


\subsection{Drosophila melanogaster}

\subsubsection{Background}

Drosophila melanogaster, commonly known as the fruit fly, has been used as a model organism for research for almost a century now, and has advanced to become one of the most valuable organisms in biological research.

Advantages of working with Drosophila include the great variety of genetic techniques available for manipulating the fruit fly's genome, which was recently sequenced in its entirety, the short time needed to generate transgenic animals, and the availability of mutant flies with defects in any of several thousand genes. Furthermore, as the development, behaviour, and morphology of Drosophila have been extensively investigated, changes in either of these criteria are relatively easy to detect. And importantly, since many genes and physiological mechanisms described in Drosophila have been shown to be highly conserved, insights won from this model are helpful in understanding similar processes in more 'advanced' organisms.

However, Drosophila also offers very practical advantages in terms of laboratory maintenance. It is a small animal (the adults are approximately $3 \mathrm{~mm}$ long), has a short life cycle of only two weeks and is thus inexpensive and easy to keep in large numbers. In the laboratory, Drosophila are kept in culture vials containing a base made up of apple agar and yeast, which supplies them with nutrition (Fig. 2B). Adult flies are transferred from one vial to the next regularly, which separates them from their offspring and ensures a constant supply of larvae of the desired developmental stage.

Figure 2 illustrates the life cycle of Drosophila. Its egg is approximately $0.5 \mathrm{~mm}$ long, and it takes about one day for the embryo to hatch into a larva [21 +/- 1 hours after egglaying (AEL)]. The larva then undergoes cycles of growth and moulting. Three days after hatching and after moulting for the second time the larva reaches the third instar stage. This period lasts for two days, after which the larva forms a cocoon and remains in a pupal stage for further four days. During this time the body undergoes remodelling, to finally hatch into the adult form. Within twelve hours after hatching, the fly then reaches fertility. The time course described above applies to Drosophila reared at $25^{\circ} \mathrm{C}$. 
At $18^{\circ} \mathrm{C}$, for example, the development takes approximately twice as long (TheFlyBaseConsortium, 2003).
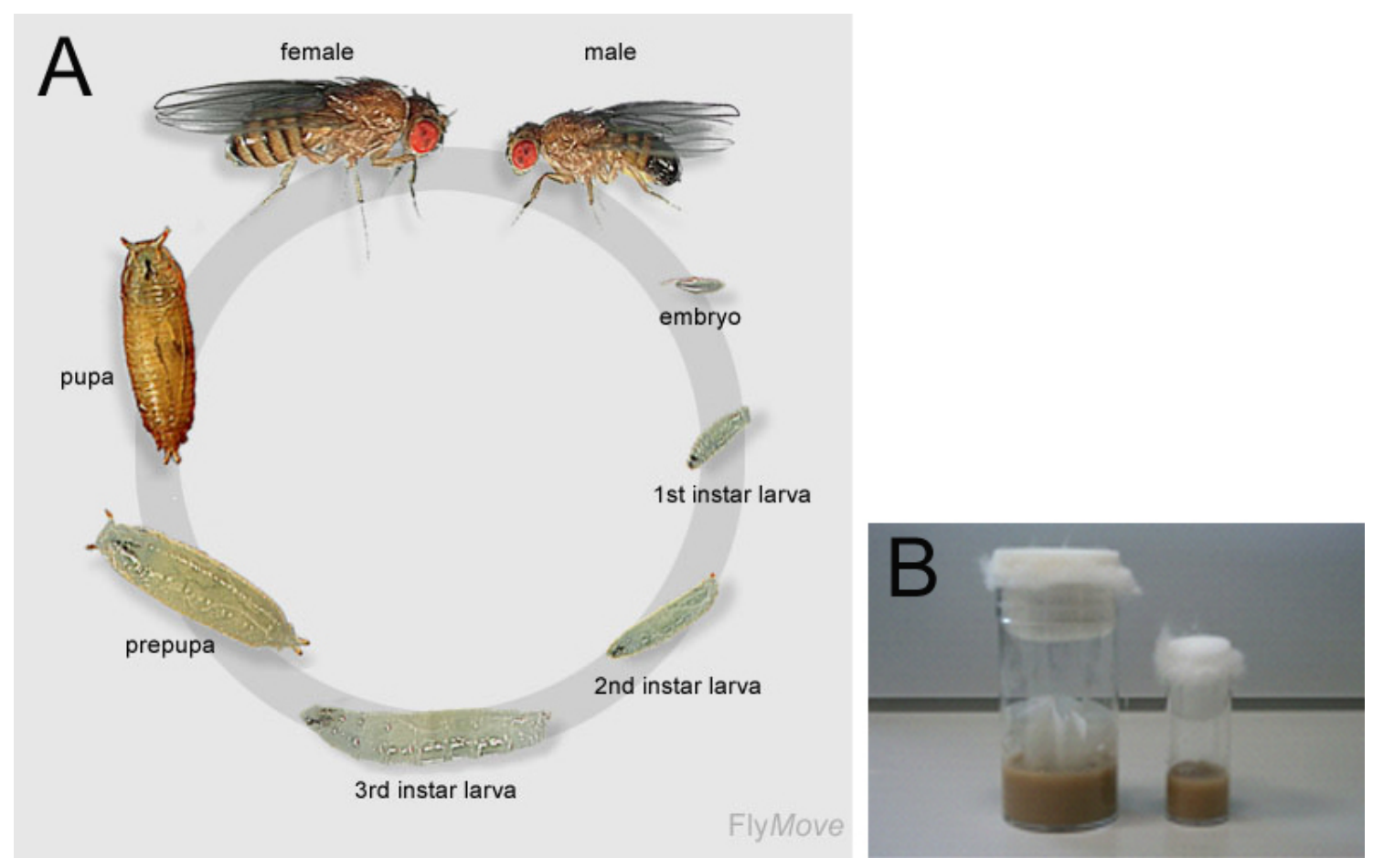

Figure 2. Drosophila melanogaster.

(A) Life cycle (Weigmann et al., 2003) and (B) culture vials (68 ml, $10 \mathrm{ml})$.

\subsubsection{Physiology and anatomy of the neuromuscular junction}

All experiments described in this report were performed on either mature embryos or late third instar larvae. The main reason for this is that at these time points the muscles have reached the maximal size of either developmental stage, and are thus most accessible. The recordings were obtained from one of the largest muscles, the ventral longitudinal muscle 6 (VLM 6), in the anterior abdominal segments A2 or A3. Figure 3 shows VLM 6 in a so-called larval fillet, which is the preparation used for electrophysiology. 

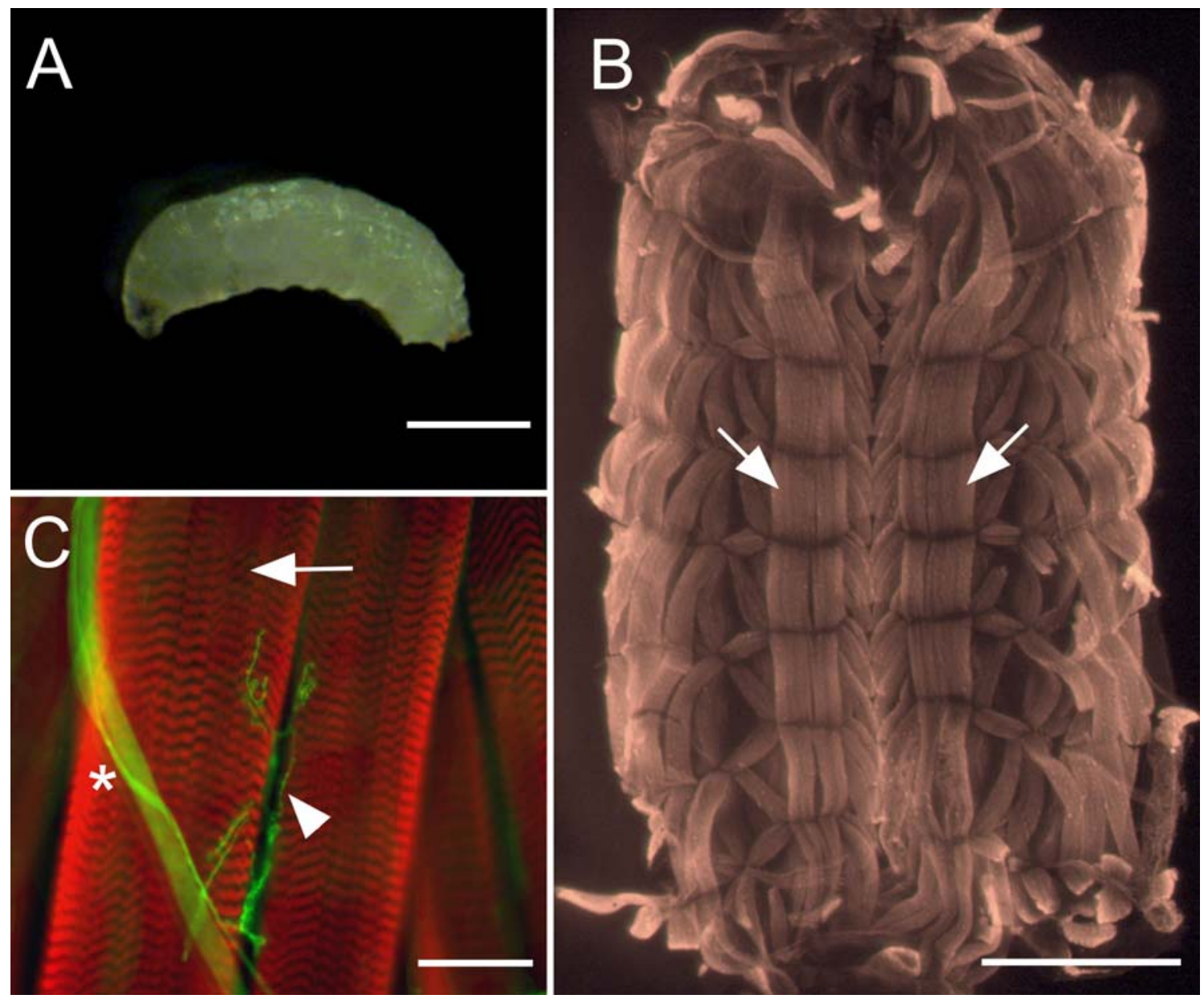

Figure 3. Larval Drosophila neuromusculature.

(A-C) Late third instar Drosophila larva. (B) Larval fillet stained with phalloidin following the removal of the CNS. The arrows identify VLM 6 in segments A3. (C) Nerve (asterisk) and axon terminals (arrowhead) of the neuronal innervation [stained with the neuronal membrane marker anti-horseradish peroxidase (HRP; green)] of VLM 6 (arrow) and 7. The muscles are stained with phalloidin (red). Scale bars, $1 \mathrm{~mm}$ in (A, B), $50 \mu \mathrm{m}$ in (C).

To my knowledge, Jan and Jan (Jan and Jan, 1976a) give the first detailed physiological description of the neuromuscular junction of full-grown Drosophila larvae. Without intending to present all their findings here, I consider it useful to give a brief summary of some of the basic anatomical and physiological properties of the larval NMJ, before summarising how these differ from the mature embryonic NMJ.

Drosophila embryos and larvae have approximately 400 striated muscle fibres, each with 10-20 nuclei aligned in longitudinal rows along the innermost surface of the fibre. The fibres of the same half segment are innervated by one nerve, which approaches from the ventral midline at the anterior border to the segment. 
From this point on, I shall concentrate on the description of VLM 6. It is approximately $400 \mu \mathrm{m}$ long, $100 \mu \mathrm{m}$ wide, and about $25 \mu \mathrm{m}$ thick. The muscle cell capacitance is in the range of 2000-5000 pF and the typical input resistance is 5-10 $\mathrm{M} \Omega$ (Stewart et al., 1994). VLM 6 is isopotential and measured with an intracellular microelectrode in "normal saline solution" has a resting membrane potential $\left(\mathrm{V}_{\mathrm{m}}\right)$ of about $-54 \mathrm{mV}$, which is neither very sensitive to changes in $\mathrm{pH}$ (between 6.8 and 7.3) nor osmolarity (between a relative tonicity of 0.88 and 1.12). $\mathrm{V}_{\mathrm{m}}$ is dependent on the extracellular concentration of sodium $\left(\left[\mathrm{Na}^{+}\right]_{\mathrm{e}}\right)$ and potassium $\left(\left[\mathrm{K}^{+}\right]_{\mathrm{e}}\right)$, but not on chloride, which is believed to distribute passively across the membrane. Furthermore, the membrane potential obeys the Goldman-Hodgkin-Katz equation for a multi-ion electrode and has an estimated ratio of permeability coefficients for sodium and potassium of 0.23 $\left(\mathrm{P}_{\mathrm{Na}} / \mathrm{P}_{\mathrm{K}}=0.23\right)$. In addition to this, Jan and Jan describe the quantal nature of presynaptic transmitter release at the Drosophila NMJ and show that release is dependant on approximately the $4^{\text {th }}$ power of $\left[\mathrm{Ca}^{2+}\right]$ e. Finally, the two authors conclude that glutamate is the excitatory transmitter at Drosophila neuromuscular junctions (Jan and Jan, 1976b).

This feature separates the NMJ of Drosophila from its cholinergic mammalian counterparts, and highlights a fundamental functional property the Drosophila NMJ has in common with the majority of excitatory synapses of the mammalian CNS. But also in terms of synaptic architecture the Drosophila NMJ shows homologies to central mammalian synaptic connections (Gramates and Budnik, 1999).

Two motoneurons [RP3 and 6/7b; (Keshishian et al., 1993)] branch out to supply the neuronal muscle innervation of both VLM 6 and 7 (Fig. 3C, 4A). In larvae, the axon terminals, or boutons, are submerged in the muscle and are surrounded by reticular invaginations of the muscle membrane, the so-called subsynaptic reticulum [SSR; Fig. 4C; (Budnik, 1996)]. Boutons of RP3 (termed type 1b) and 6/7b (type 1s) are 3-5 $\mu \mathrm{m}$ and 1-3 $\mu \mathrm{m}$ in diameter and posses on average 41 and 7 individual synapses respectively (Fig. 4). In total, VLM 6 receives input from approximately 450-650 individual monadic synapses, where one presynaptic site displays a continuous junction with just a single postsynaptic site, and of which a fraction may be physiologically silent under normal operating conditions (Atwood et al., 1993; Atwood and Wojtowicz, 
1999). The individual presynaptic membranes, found in tight planar alignment with postsynaptic membranes, are comparably large $(400-600 \mathrm{~nm})$, and frequently display electron-dense projections termed T-bars that are believed to demark individual active zones within the presynaptic membrane [Fig. 1B, 4C; (Atwood et al., 1993)] and are also found in other dipteran insects (Trujillo-Cenoz, 1969; Feeney et al., 1998).
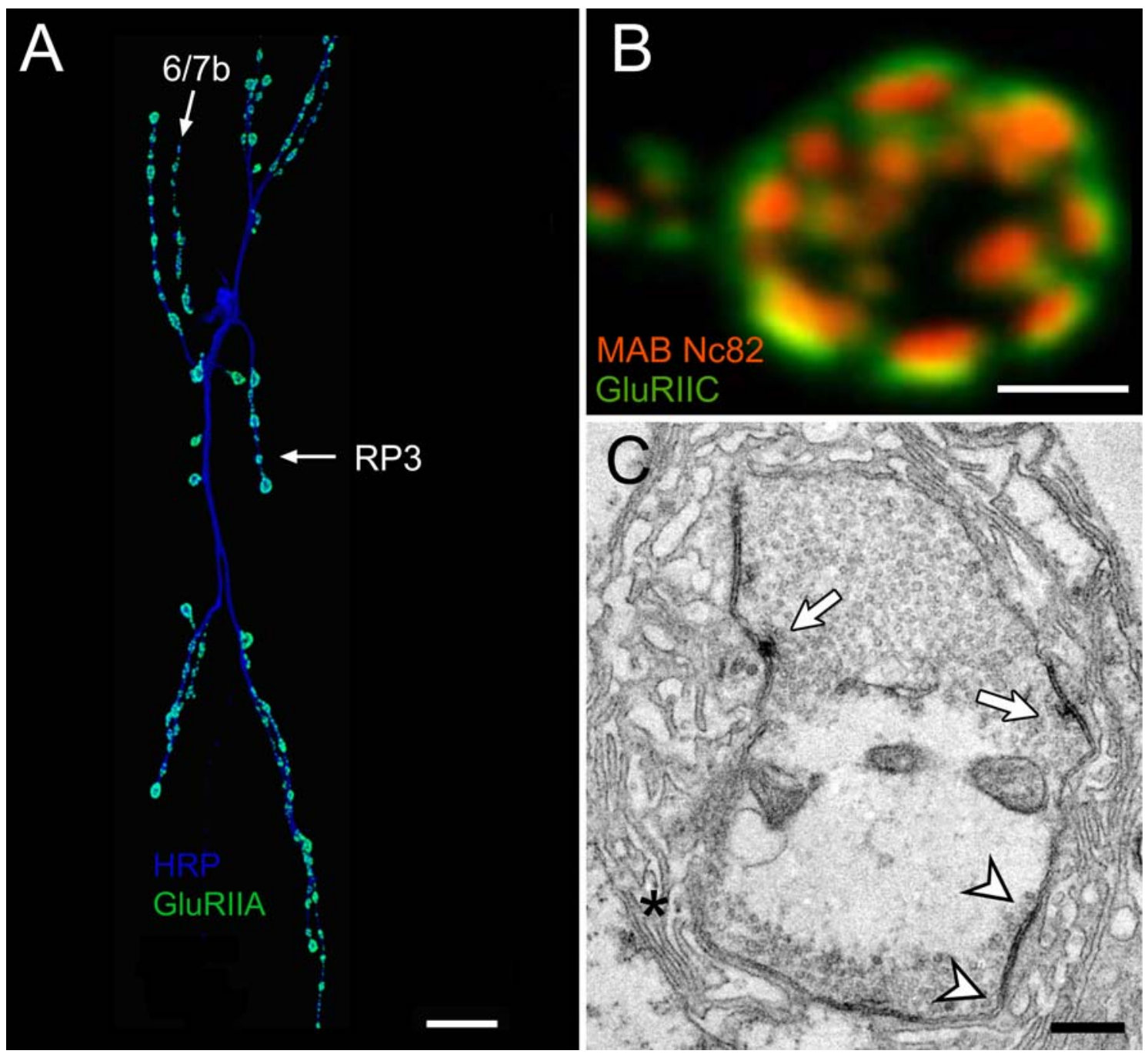

Figure 4. The structure of synaptic connections at the larval NMJ.

(A) The neuronal innervation of VLM 6 and 7 consists of the motoneurons RP3 and 6/7b (both labelled by anti-HRP in blue). The receptor fields are marked by an antibody against the glutamate receptor subunit GluRIIA (green). (B) A single bouton of RP3 demonstrates the alignment of presynaptic sites [labelled by the monoclonal antibody (MAB) Nc82] and postsynaptic sites (stained against the receptor subunit GluRIIC). (C) An electron micrograph shows the ultrastructure of a bouton surrounded by the SSR (asterisk). Synapses can be identified by electron-dense pre- and postsynaptic membranes, with (arrows) and without (arrowheads) T-bars. The synaptic vesicles are distributed around the cortex of the bouton, and the three large electron-dense structures in the centre are mitochondria (Kittel et al., 2006b). (A, B) Courtesy of W. Fouquet. Scale bars, $20 \mu \mathrm{m}$ in (A), $1 \mu \mathrm{m}$ in (B), and $250 \mathrm{~nm}$ in (C). 
The postsynaptic site in turn, contains evolutionarily conserved ionotropic glutamate receptors and other ion channels, arranged in a well defined electron-dense thickening called the postsynaptic density (Atwood et al., 1993; Jia et al., 1993). The PSD is enriched in signalling molecules, scaffolding proteins, and plays a major role in clustering and stabilising the receptors and ion channels (Kennedy, 1997; Rodesch and Broadie, 2000). The synaptic extracellular matrix, located in the cleft between pre- and postsynaptic partners keeps both membranes in precise alignment and 10-20 nm apart, in contrast to vertebrate neuromuscular synapses where the synaptic cleft is significantly wider than at central synapses (Peters et al., 1991; Burns and Augustine, 1995; Prokop and Meinertzhagen, 2006).

In the Drosophila embryo, muscles develop from unfused myoblasts to contractile, syncytial muscle fibres with all the characteristics of the mature larval muscles within 12 hours at $25^{\circ} \mathrm{C}$ (Bate, 1990). Membrane electrogenesis begins in the mid-embryonic stages (approx. 13 hours AEL), and continues so that by the late embryonic stage (approx. 21 hours AEL) the macroscopic currents observed in mature larval muscles have developed (Broadie and Bate, 1993b). VLM 6 of the mature embryo has an average diameter of $10-20 \mu \mathrm{m}$ and an average length of $40-80 \mu \mathrm{m}$. The input resistance of the muscle is in the range of $200 \mathrm{M} \Omega$ to over $1 \mathrm{G} \Omega$ and the cell capacitances are between 10 and $30 \mathrm{pF}$, increasing with developmental age (Broadie, 2000).

The embryonic boutons have a diameter of up to $1 \mu \mathrm{m}$, and in contrast to the submerged neuronal terminals of larvae, one side of each bouton faces the haemolymph and is merely covered by basement membrane (Prokop, 1999). The SSR only begins to develop after embryogenesis has completed (Prokop and Meinertzhagen, 2006). There are roughly 10 times less boutons at the embryonic neuromusculature, and each terminal contains 3 to 10 times fewer individual synapses (Schuster et al., 1996a). Despite these differences in the architecture of the NMJ, the characteristics of individual neuromuscular synapses are considered similar between embryo and larva (Prokop, 1999).

The wide array of genetic techniques available for manipulating the fruit fly genome and the accessibility of the neuromuscular junction (NMJ) to optical and physiological recordings can be combined to elucidate the roles of individual molecules in synaptic 
transmission. As the structure and physiology of both the embryonic and larval NMJ are well documented, changes in either of these criteria can be detected with relative ease (Broadie and Bate, 1993a, 1993b; Prokop, 1999; Singh and Wu, 1999; Prokop and Meinertzhagen, 2006). Notably, boutons can be added or removed in an activitydependent manner, or in response to developmental cues, thereby leading to long-lasting changes in synaptic strength (Petersen et al., 1997; Davis and Goodman, 1998; Sigrist et al., 2000; Sigrist et al., 2002; Sigrist et al., 2003). Furthermore, such modulations also affect the compositional complexity of individual synapses. For example, increases in synaptic strength have been correlated with an elevated expression of presynaptic Tbars (Renger et al., 2000; Sigrist et al., 2002). Thus, the Drosophila NMJ shows a dynamic plasticity, also exhibited by many central mammalian synapses. It is this developmental and activity-dependent modulation of synaptic growth and function that sets the foundation for this research project, and which I will describe in greater detail in the following chapter.

\subsubsection{Glutamate receptor subunit expression influences synaptic strength and morphology at the Drosophila NMJ}

There is an approximately 100-fold increase in muscle surface area as Drosophlia larvae develop from first to third instar (Petersen et al., 1997). Parallel to this, the neuronal muscle innervation grows immensely and increases the number of boutons, synapses per bouton (Schuster et al., 1996a), and vesicles within each bouton (Prokop, 1999). Thus, changes in synaptic strength and morphology occur at high rates during the development of the larval NMJ. Activity-dependent changes in synaptic function have also been conclusively demonstrated at the neuromuscular junction. Activity-mutants, such as the double mutant for potassium channel subunits ether a go-go (eag) and shaker (sh), or the cAMP-phosphodiesterase mutant dunce, have been used as models to show that the Drosophila NMJ exhibits increased structural growth and transmission strength in response to an increase in neuronal activity (Budnik et al., 1990; Zhong et al., 1992).

In addition to this, it was demonstrated that increases in neuronal activity can also trigger local postsynaptic protein synthesis at the Drosophila NMJ. This, in turn, is 
necessary and sufficient for structural and functional long-term strengthening, through the synthesis of additional synapses (Sigrist et al., 2000; Sigrist et al., 2002). These results are especially intriguing, when considering that local sub-synaptic protein synthesis has been shown to be involved in learning and memory processes in both invertebrates (Casadio et al., 1999; Martin et al., 2000; Menon et al., 2004) and mammals (Wu et al., 1998; Wells et al., 2000; Steward and Schuman, 2001).

In the context of translationally-mediated NMJ strengthening, the analysis of the relevant substrate-mRNAs and the synthesised proteins is of further interest. A number of ionotropic glutamate receptor subunits, belonging to the family of non-NMDA type receptors, are expressed at the Drosophila NMJ. Two of these subunits, encoded by adjacent genes, are GluRIIA and GluRIIB (Schuster et al., 1991; Petersen et al., 1997; DiAntonio et al., 1999). In addition, the GluRIII (also referred to as GluRIIC) receptor subunit was recently identified (Marrus et al., 2004). The observation that a deletion of the gluRIIC gene causes embryonic lethality, and that the number of neuromuscular glutamate receptors is severely diminished in GluRIIC hypomorphs, suggests that GluRIIC is a required subunit. In contrast, neither GluRIIA nor GluRIIB is essential for viability, though simultaneous deletion of both corresponding genes causes embryonic lethality and a complete loss of glutamate receptors from the NMJ (DiAntonio et al., 1999). Based on these results, it was hypothesised that glutamate receptors at the Drosophila NMJ are composed of GluRIIC in combination with either GluRIIA or GluRIIB (Marrus et al., 2004).

Two further subunits, GluRIID and GluRIIE, have now been identified (Featherstone et al., 2005; Qin et al., 2005). These genomic neighbours are structurally similar to each other, but differ from the other three. Eliminating the expression of either GluRIID or IIE, results in embryonic lethality. Furthermore, it was observed that the synaptic expression of GluRIIA, IIB and IIC is fully dependent on both GluRIID and IIE expression, and that vice versa the synaptic expression of GluRIID requires GluRIIA/IIB and GluRIIC. Thus, the formation of synaptic glutamate receptor complexes requires the simultaneous presence of four structurally diverse receptor subunits (Qin et al., 2005). One likely scenario is that the glutamate receptor population at the Drosophila NMJ is a mixture of IIA, IIC, IID, IIE and IIB, IIC, IID, IIE complexes, though the precise stoichiometry of the receptors remains to be determined. 
Importantly, the above mentioned plasticity mutants (eag Sh and dunce), which are characterised by a phenotype of increased neuronal activity, have large aggregates of both translational components, and GluRIIA mRNA, localised within sub-synaptic compartments, associated with the SSR (Sigrist et al., 2000).

Moreover, genetic elevation of sub-synaptic protein synthesis, leads to increased junctional outgrowth, and is also associated with a persistent enhancement of signal transmission (Sigrist et al., 2000). In this situation GluRIIA, but not GluRIIB, mRNA can be detected in the SSR. This evidence strongly suggests an involvement of the IIA subunit in activity-dependent strengthening of the NMJ. Furthermore, ultrastructural analyses demonstrate that the increased synaptic expression of GluRIIA (also achieved by direct transgenic overexpression) triggers the formation of synapses harbouring presynaptic T-bars. The synapses appear to have defined space requirements, which are met by the down-regulation of the cell-adhesion molecule Fasciclin II, thereby leading to the growth of additional boutons, and a corresponding enhancement in the strength of junctional signal transmission (Sigrist et al., 2002).

Conversely, such a strengthening of the NMJ can be suppressed by the downregulation of GluRIIA expression. Genetic experiments have shown that this can be accomplished through either a reduced gluRIIA gene copy number, or transgenic overexpression of GluRIIB (Sigrist et al., 2002). Thus, in terms of synaptic plasticity, these two glutamate receptor subunits have antagonistic effects, and it seems likely that at the Drosophila $\mathrm{NMJ}$, the activity of receptors can regulate a retrograde signal which provides the synaptic system with mechanisms to generate plasticity.

But also in terms of signal transmission, receptor ion channels containing the GluRIIA subunit differ from those composed, at least in part, of the GluRIIB subunit (DiAntonio et al., 1999). Intracellular current-clamp recordings demonstrated that larvae lacking the IIA subunit show a lower postsynaptic response to single vesicle fusions (quantal size) than mutants of the gluRIIB gene. Though this may also be attributable to differences in receptor density, recordings from outside-out patches, isolated from the larval muscle membrane, illustrated that GluRIIB containing channels have faster desensitisation kinetics (average time constant of $2 \mathrm{~ms}$ ) than those containing GluRIIA [average time 
constant of $19 \mathrm{~ms}$; (DiAntonio et al., 1999)]. Non-NMDA type ion channels of arthropods have a high unitary conductance compared to their vertebrate counterparts (Cull-Candy and Parker, 1982). In Drosophila larvae, the single channel conductance of extrasynaptic neuromuscular glutamate receptors has been measured as approximately 120 pS (Heckmann and Dudel, 1995; DiAntonio et al., 1999), and the unitary conductance of embryonic synaptic receptors has been estimated as 115 pS (Nishikawa and Kidokoro, 1995). It has also been suggested that two synaptic receptor populations with different unitary conductance may exist in the embryo (Kidokoro and Nishikawa, 1994), and that these could reflect low conductance receptors containing GluRIIB, and higher conductance receptors lacking GluRIIB (Chen and Featherstone, 2005). However, the absence of GluRIIB from extrasynaptic larval receptors does not influence the unitary current amplitude (DiAntonio et al., 1999).

Interestingly, it appears that decreases in quantal size are compensated by the presynapse, in terms of the number of vesicles released per presynaptic action potential [quantal content; (Davis and Goodman, 1998; DiAntonio et al., 1999; Paradis et al., 2001; Yoshihara et al., 2005)]. Such a retrograde homeostatic compensation has also been suggested to function at excitatory vertebrate synapses (Turrigiano et al., 1998). 


\section{$3 \underline{\text { Methods }}$}

\subsection{Principles of operation}

\subsubsection{Voltage clamping with two microelectrodes}

Most electrophysiological data presented in this thesis were obtained from intracellular recordings using the two-electrode voltage clamp technique.

The principle of a voltage-clamp is to hold the membrane potential $\left(\mathrm{V}_{\mathrm{m}}\right)$ of the cell of interest constant (to 'clamp' it at a command potential). When the membrane potential deviates from this command potential $\left(\mathrm{V}_{\mathrm{cmd}}\right)$, due to current flowing through the membrane (e.g. via open ion channels), the clamp circuitry applies an equal current of inverted polarity to the cell, and it is this compensatory current which the investigator measures.

Generally, the interest is focused on the membrane conductance, as this is directly proportional to ion channel activity. But because the conductance cannot be measured in a straightforward manner, current is recorded instead. And as long as the membrane potential remains constant, or in practice shows only a very small, brief error in response to a voltage step, the current will be linearly proportional to the conductance, and thus the activity of ion channels.

The two-electrode voltage clamp (TEVC), as applied here, requires two microelectrodes inserted into the cell. To implement this, each of two $\mathrm{Ag} / \mathrm{AgCl}$ (silver/silver-chloride) electrodes is placed in a sharp glass micropipette, filled with an electrolyte solution. One is the voltage-sensing microelectrode (ME1) and the other is the current-passing microelectrode (ME2). ME2 is connected to a voltage source which contains a voltageclamp gain control. This gain control (in units of $\mathrm{V} / \mathrm{V}$ ) determines the response speed, and the accuracy of the clamp at the steady-state level. It allows the investigator to adjust how many volts the output will change for each volt difference between $V_{c m d}$ and $\mathrm{V}_{\mathrm{m}}$. Thus, to a certain extent the gain control can be used to compensate for the time required to charge the cell membrane capacitance $\left(\mathrm{C}_{\mathrm{m}}\right)$, and for both the pipette 
resistance of ME2 $\left(R_{p 2}\right)$, and the cell membrane $\left(R_{m}\right)$, in order to ensure the fastest possible clamp response to a voltage step (without inducing excess noise).

The following equations describe how the clamp error depends on $\mathrm{R}_{\mathrm{p} 2}$ and $\mathrm{R}_{\mathrm{m}}$ and how the gain can be used to minimise the error (TheAxonGuide, 1993). One way of simulating the ability of the voltage clamp to follow a change in membrane conductance is to apply a step change in $\mathrm{V}_{\mathrm{cmd}}$ (Finkel and Gage, 1985). After a step change in $\mathrm{V}_{\mathrm{cmd}}$, $\mathrm{V}_{\mathrm{m}}$ is governed by:

$V_{m}=V_{c m d} \frac{\mu K}{\mu K+1}$

where $\mu$ is the gain of the clamp amplifier and $\mathrm{K}$ is the attenuation of the amplifier due to $\mathrm{R}_{\mathrm{m}}$ and the resistance of $\mathrm{R}_{\mathrm{p} 2}$. Furthermore:

$K=\frac{R_{m}}{R_{m}+R_{p 2}}$

Therefore, the larger the product of $\mu \mathrm{K}$, the lower the voltage error will become. If $\mu$ was infinite, or $\mathrm{R}_{\mathrm{p} 2}$ was zero, the response would approach the ideal case. There is however a limit as to how much gain can be applied while conserving a useful signal. When $\mu$ is very large, oscillations are introduced (mainly through coupling capacitance), which will bury the signal, so that in this scenario the drawbacks outweigh the advantages of having a very short response time. The other approach is to maximise $\mathrm{K}$, by using a current-passing microelectrode with as small a resistance as possible (ideally of the same, or lower resistance than that of the membrane). However, as micropipettes with a low resistance tend to be blunt, this increases the risk of damaging the cell membrane. Thus, a careful balance has to be achieved when considering these factors.

Other limitations of the two-electrode voltage clamp, which I will not discuss in more detail include the limited bandwidth of ME1, capacitive coupling between the two MEs, 
and non-ideal phase shifts in the membrane. Most likely the major disadvantage of the TEVC is the complexity arising from two microelectrodes applied to the same cell. Nonetheless, the two-electrode voltage clamp offers a number of powerful advantages, which have helped it become a very popular investigation technique for preparations such as the late third instar larval Drosophila NMJ (Wu and Haugland, 1985).

The TEVC has low noise levels compared to voltage clamping with a single microelectrode of comparable resistance in the discontinuous mode (dSEVC). And importantly, the TEVC has a better temporal resolution. Steady-state conditions can be re-established rapidly after a sudden change in membrane resistance, as would be the case following the opening of ion channels (Finkel and Redman, 1985). Thus, together with a very high current-passing capacity, right up to the $\mathrm{mA}$ range (Axoclamp-2B, 1997), large currents can be recorded in TEVC with acceptable voltage errors. Exploiting these features allows an investigator to clamp very large cells, with low input resistance, such as the larval VLM 6.

A schematic representation of the two-electrode voltage clamp in operation at the Drosophila NMJ is shown in Figure 5. In addition to the features described above, this setup includes a suction electrode (SE), into which the nerve innervating the segment of interest can be pulled, to apply voltage pulses to it. This triggers action potentials which propagate down the motoneurons, invade the presynaptic terminals, leading to $\mathrm{Ca}^{2+}$ influx and synaptic vesicle exocytosis. The consequence can be measured as evoked excitatory junctional currents (eEJCs) postsynaptically by the voltage clamp (Fig. 6A). The eEJCs reflect the compound EJC of both motoneurons innervating VLM 6. 

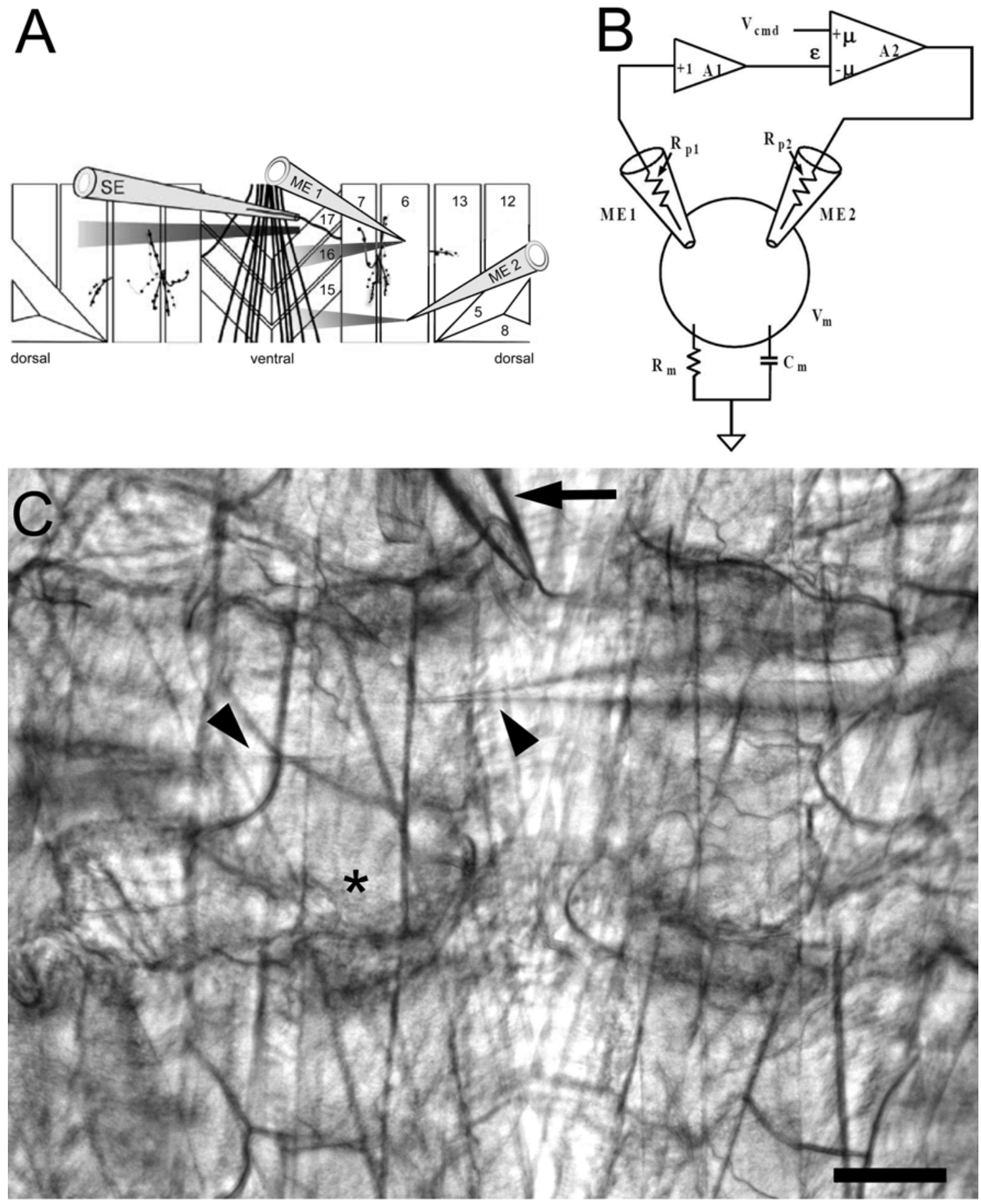

Figure 5. The two-electrode voltage clamp in operation at the larval NMJ.

(A) Schematic representation of the TEVC [modified from (Pawlu et al., 2004)]. The suction electrode triggers nerve-evoked junctional currents. (B) Simplified circuit diagram (TheAxonGuide, 1993). ME1 is connected to a unity-gain buffer amplifier (A1) that records $\mathrm{V}_{\mathrm{m}}$. A high-gain differential amplifier $(A 2$; gain $=\mu)$ then compares this value with $V_{c m d}$. The output of $\mathrm{A} 2$ is proportional to the difference $(\varepsilon)$ between $\mathrm{V}_{\mathrm{m}}$ and $\mathrm{V}_{\mathrm{cmd}}$ and forces current to flow through ME2 into the cell. The polarity of $\mu$ in A2 is set so that the current in ME2 reduces $\varepsilon$. (C) Operating configuration of the TEVC at the larval NMJ. Two microelectrodes (arrowheads) are inserted into VLM 6 (asterisk), and the innervating nerve is placed in the suction electrode (arrow). Scale bar, $100 \mu \mathrm{m}$. 
The second main phenomenon the voltage-clamp can measure in this preparation is the postsynaptic response to single quanta of presynaptically released transmitter (Fig. 6B, 8D). These miniature excitatory junctional currents (mEJCs or minis) are believed to reflect spontaneous vesicle fusion events (Del Castillo and Katz, 1954; Katz, 1969). Traditionally, the amplitude of the postsynaptic current generated by the minis has been taken as a measure of the size of the receptor field, or the nature of the ion channels coupled to the receptors, which is referred to as quantal size.

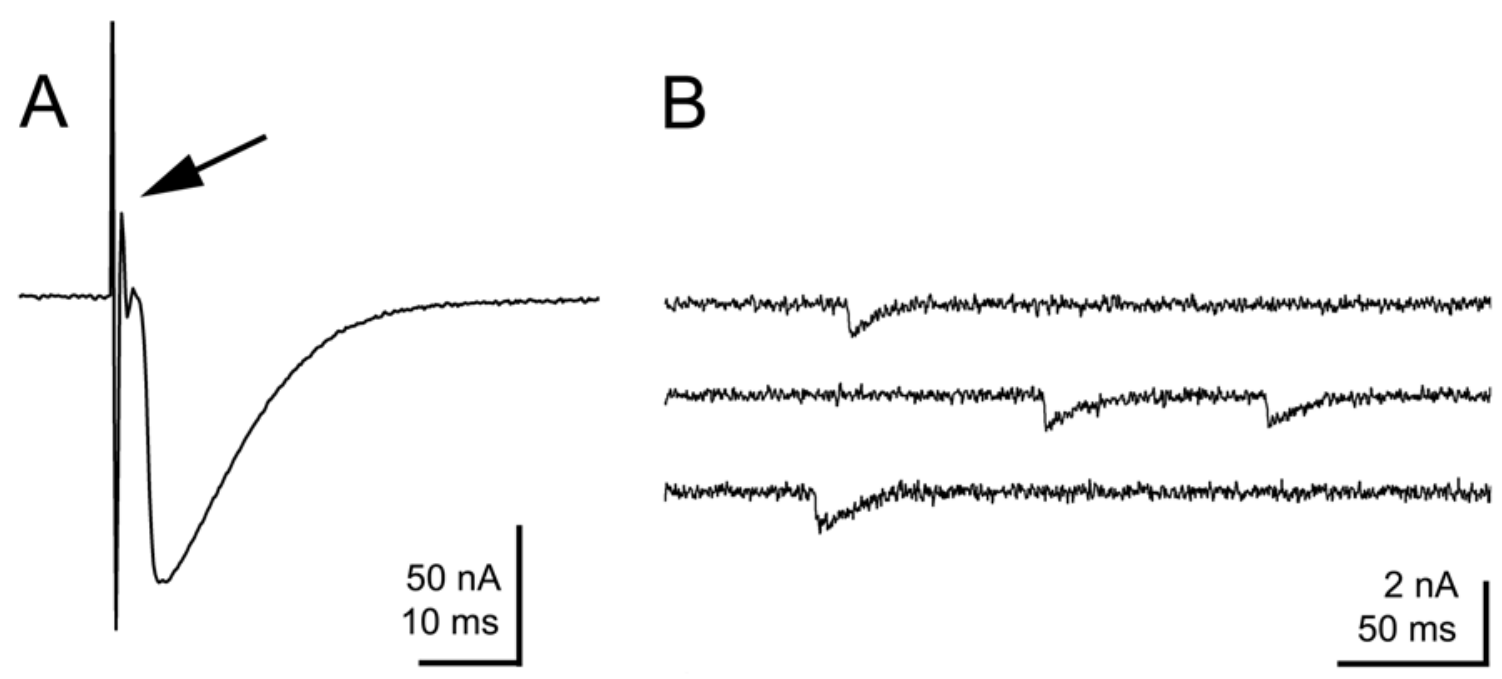

Figure 6. Example traces of two-electrode voltage clamp recordings.

(A) Nerve-evoked excitatory junctional current and (B) miniature excitatory junctional currents recorded from VLM 6 of the larval NMJ in TEVC. The arrow indicates the artefact following nerve stimulation (Kittel et al., 2006b).

Finally, when combining measurements of eEJCs and mEJCs, data can be extracted concerning the number of vesicles released per action potential. This quantal content gives insight into the presynaptic strength. 


\subsubsection{Voltage clamping with a single patch pipette}

Several experiments were performed in the continuous single-electrode voltage clamp (sSEVC) configuration.

To implement the whole-cell patch clamp, a blunt, smooth, low-resistance glass pipette is sealed by suction to the surface of the cell membrane, yielding a seal resistance in the G $\Omega$ range (Neher, 1981). The very significant result of this cell-attached mode is that the high resistance of the 'giga-seal' reduces the background noise of the recording by an order of magnitude, to deliver single channel-resolution [Fig. 8C; (Sigworth and Neher, 1980)]. For the whole-cell configuration, the membrane patch enclosed within

the pipette tip is ruptured, and the electrolyte solution in the pipette forms an electrical continuity with the cell (Hamill et al., 1981).

Whereas in TEVC and ASEVC the voltage at the tip of the pipette is controlled, the cSEVC controls the voltage at the top of the pipette. As a consequence, the series resistance $\left(\mathrm{R}_{\mathrm{s}}\right.$; the sum of the pipette resistance and the residual resistance of the ruptured patch) can introduce significant errors when the current flowing through the membrane is large. Even though $\mathrm{R}_{\mathrm{s}}$ can be compensated for to about $80 \%$, the cSEVC cannot be used to reliably record the large ionic currents of the entire larval Drosophila muscle. However, whole-cell patch clamp recordings can be obtained from the smaller embryonic muscle following enzymatic cleansing of its surface [Fig. 7, 8D; (Broadie and Bate, 1993a)]. 

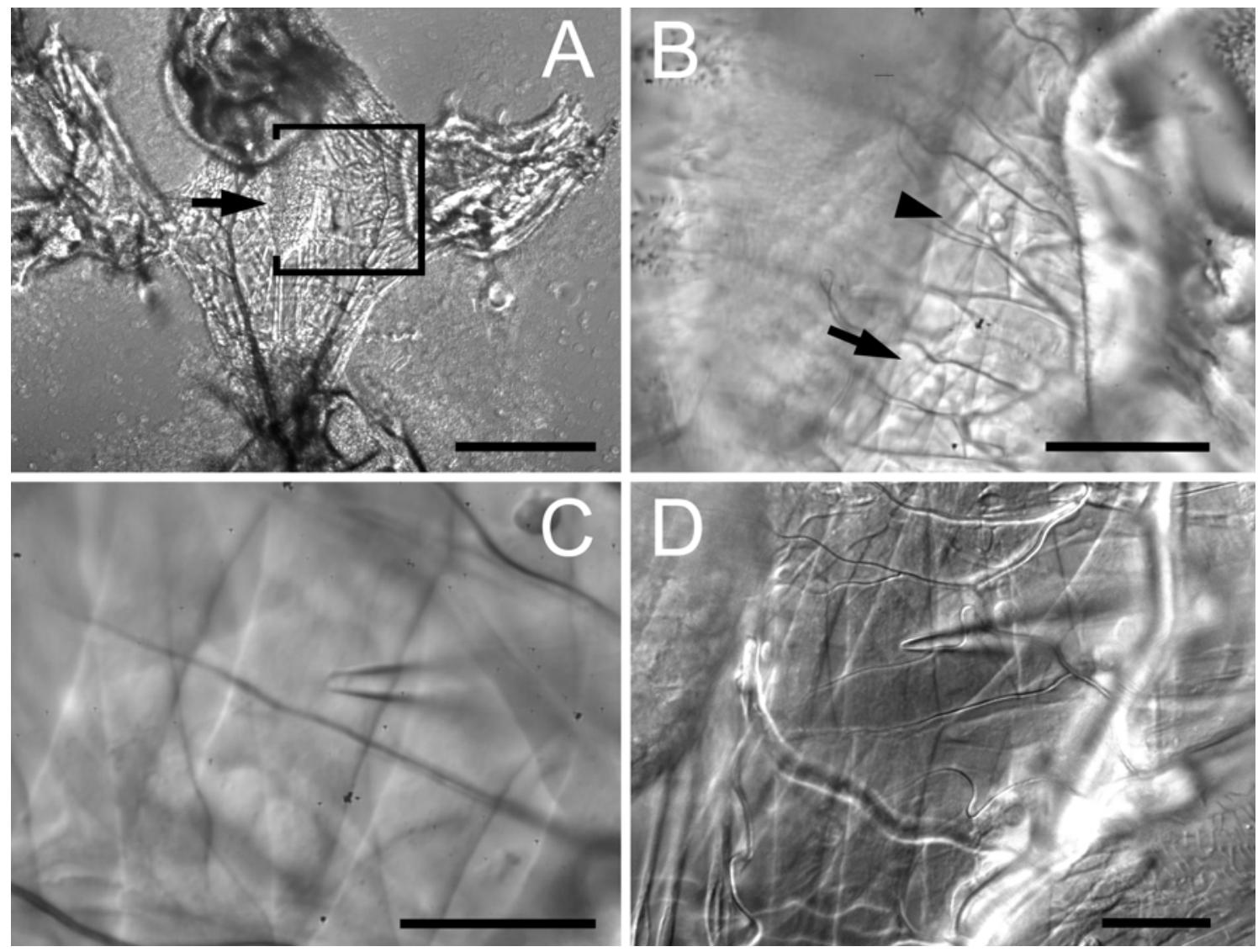

Figure 7. Embryonic preparation for patch-clamp recordings.

(A) Preparation of a Drosophila embryo to expose the ventral longitudinal muscles. The arrow points at the CNS and the bracket represents the approximate location of the magnified region in (B). (B) VLM 6 in anterior abdominal segments A2 (arrowhead) and A3 (arrow). (C, D) A patch-clamp pipette positioned for whole-cell recordings from VLM 6. Scale bars, $100 \mu \mathrm{m}$ in (A), $50 \mu \mathrm{m}$ in (B), and $20 \mu \mathrm{m}$ in (C, D).

The cSEVC can also be implemented to record local postsynaptic currents from the larval muscle (Kurdyak et al., 1994; Heckmann and Dudel, 1998; Pawlu et al., 2004). In brief, a macro-patch pipette with a large, smooth opening of roughly 5-10 $\mu \mathrm{m}$ is placed on the muscle enclosing an area of neuronal innervation, to record postsynaptic signals extracellularly (Fig. 8A). In principle, such focal recordings correspond to a 'loose' cellattached patch clamp configuration (Dudel, 1981). Although the focal electrode does not record the entire muscle response, it delivers an excellent resolution of local events (Fig. 8B) compared to voltage-clamping with two intracellular microelectrodes, and can be used to singularly record from, and differentiate between the two innervations of VLM 6 (Kurdyak et al., 1994). 
A

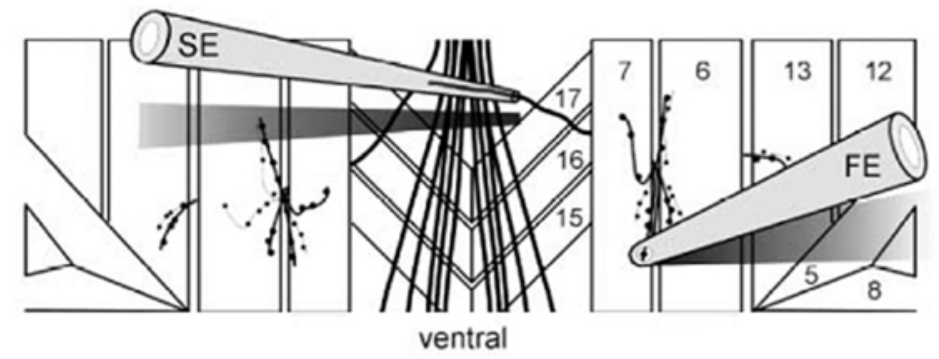

C
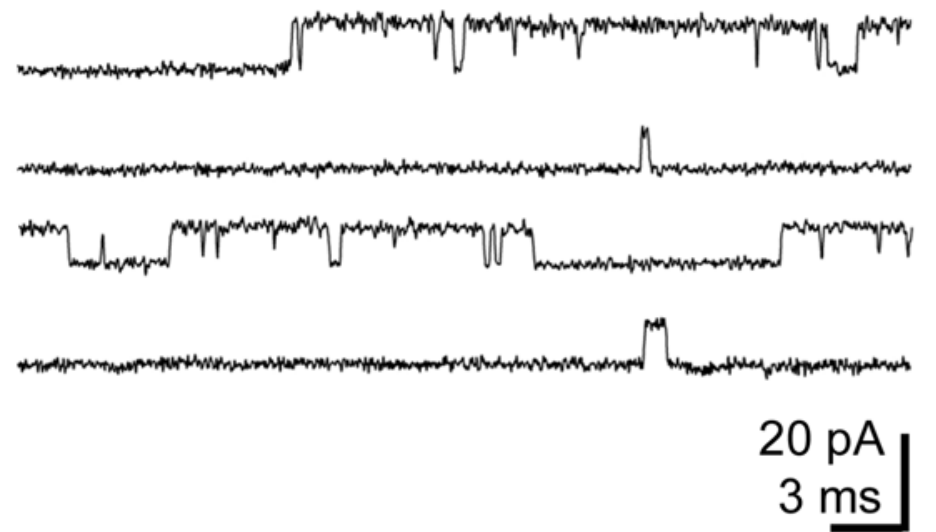

B

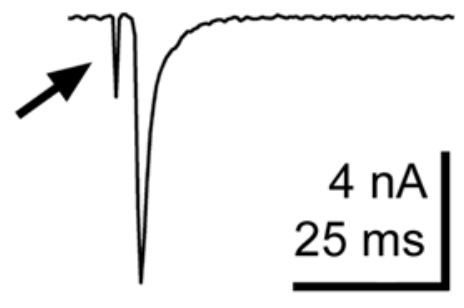

$\mathrm{D}$

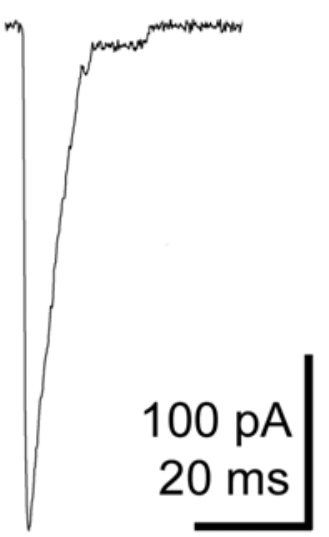

Figure 8. Example traces of patch clamp recordings.

(A, B) Larval loose-patch recordings. (A) Schematised setup for focal recordings from Drosophila larvae (Pawlu et al., 2004). The focal electrode (FE) is placed on the muscle over selected terminals, to record (B) local postsynaptic currents following nerve-stimulation (arrow shows stimulation artefact). (C, D) Embryonic patch-clamp recordings. (C) Single channel resolution of example traces recorded from VLM 6 in the cell-attached configuration $\left(\mathrm{V}_{\mathrm{cmd}}-90\right.$ $\mathrm{mV}$ ). (D) Miniature excitatory junctional current recorded in whole-cell mode. 


\subsection{Details}

\subsubsection{TEVC recordings}

Intracellular recordings were obtained at $22^{\circ} \mathrm{C}$ from VLM 6 in anterior abdominal segments A2 and A3, of late male third instar larvae. To expose the ventral muscles, the head and tail were pinned down, and dissection scissors were used to make a longitudinal incision along the dorsal midline. The cut dorsal edges were then pinned down to remove the internal organs (gut, fat body), and finally the segmental nerves were severed near the ventral nerve chord to remove the CNS and eliminate CNSmediated spontaneous transmission. The larvae were dissected in ice-cold, $\mathrm{Ca}^{2+}$-free haemolymph-like saline [HL-3; (Stewart et al., 1994)]. Composition of the HL-3 solution was (in $\mathrm{mM}$ ): $\mathrm{NaCl} 70, \mathrm{KCl} 5, \mathrm{MgCl}_{2} 20, \mathrm{NaHCO}_{3} 10$, trehalose 5, sucrose 115, HEPES 5, $\mathrm{pH}$ adjusted to 7.2. Larval fillets were rinsed with HL-3 saline containing $1 \mathrm{mM} \mathrm{Ca}^{2+}$, before being transferred to the recording chamber where the larval NMJ was visualized with a fixed-stage upright microscope (Olympus, BX51WI; 40x water-immersion lens).

Both miniature and evoked postsynaptic currents were recorded in $1 \mathrm{mM}$ extracellular $\mathrm{Ca}^{2+}$ in the TEVC mode (AxoClamp 2B, Axon Instruments, USA) using sharp micropipettes (borosilicate glass with filament, $1.5 \mathrm{~mm}$ outer diameter) filled with $3 \mathrm{M}$ KCL to give resistances of 15-35 M (Qin et al., 2005; Rasse et al., 2005; Schmid et al., 2006), 12-25 M $\Omega$ (Kittel et al., 2006b; Wagh et al., 2006), or 8-21 M $\Omega$ (Besse et al., 2006). All cells selected for analysis had resting potentials between -50 and $-70 \mathrm{mV}$, and input resistances of $\geq 4 \mathrm{M} \Omega$.

For stimulation, the cut end of the segmental nerve was pulled into a fire-polished suction electrode (10-15 $\mu \mathrm{m}$ inner diameter) to pass brief (300 $\mu \mathrm{s})$ depolarizing pulses (npi stimulus generator and isolation unit). To ensure the stable recruitment of both innervating motoneurons, the amplitude of the pulse was set to about 1.5 times the amplitude needed to recruit both motoneurons (in practice usually $-12 \mathrm{~V}$ ). The clamp was tuned such that it responded to a voltage step from -60 to $-70 \mathrm{mV}$ with settling times of $1 \mathrm{~ms}$ (Qin et al., 2005; Rasse et al., 2005) to $1.5 \mathrm{~ms}$ (Besse et al., 2006; Kittel et al., 2006b; Wagh et al., 2006) for mEJCs, and 500-750 $\mu \mathrm{s}$ for eEJCs. This gave 
voltage errors of maximally $4 \mathrm{mV}$ for eEJCs of approximately $100 \mathrm{nA}$. Both eEJCs (voltage clamp at $-60 \mathrm{mV}$ ) and $\mathrm{mEJCs}$ (voltage clamp at $-80 \mathrm{mV}$ ) were recorded at a sampling rate of $5 \mathrm{kHz}$ and low-pass filtered at $1 \mathrm{kHz}$. The holding current never exceeded $\pm 10 \mathrm{nA}$.

The quantal content was roughly estimated by dividing the average eEJC amplitude through that of the average $\mathrm{mEJC}$, recorded from the same cell. Miniature EJC recordings lasted for $90 \mathrm{~s}$, and $0.2 \mathrm{~Hz}$ stimulation protocols included 15 (Besse et al., 2006) or 20 traces/cell. Paired-pulse recordings consisted of 20 (Schmid et al., 2006) or 10 traces/interval/cell (Kittel et al., 2006b). During paired-pulse stimulation, $4 \mathrm{~s}$ of rest were left between paired-pulses. The amplitude of the second pulse in $10 \mathrm{~ms}$ interpulse recordings was measured from the peak to the point of interception with the extrapolated first pulse. High-frequency stimulation followed $30 \mathrm{~s}$ of rest. The rise time and decay time constant $(\tau)$ were obtained from the average event of the corresponding recording. The rise time was measured from 10 to $90 \%$ of the maximum amplitude, and the decay was fit with a single exponential function from $60 \%$ of the peak. The event analysis was performed with pClamp 9 (Axon Instruments), and stimulation artefacts of eEJCs were removed for clarity.

\subsubsection{Focal recordings}

Focal electrode recordings were obtained at room temperature from VLM 6 of late third instar larvae as formerly reported (Pawlu et al., 2004). Recordings following EGTAAM application lasted for $5000 \mathrm{~s}$ and were acquired in modified HL-3, consisting of (in $\mathrm{mM}$ ): $\mathrm{NaCl} 82.75, \mathrm{KCl} 5, \mathrm{MgCl}_{2}$ 1.5, $\mathrm{NaHCO}_{3}$ 10, trehalose 5, sucrose 115, HEPES 5, $\mathrm{CaCl}_{2}$ 1, 0.1 EGTA-AM (Calbiochem, diluted in dimethylsulfoxide (DMSO) with $20 \%$ (w/v) Pluronic F-127, Molecular Probes), pH adjusted to 7.2. The macro-patch pipette was backfilled with the modified HL-3 prior to recordings. Nerve simulation was applied at $0.2 \mathrm{~Hz}$. The initial and final amplitudes, as well as the representative traces, were obtained by averaging 20 responses. 


\subsubsection{Whole-cell patch clamp recordings}

Morphologically and temporally staged mature embryos (approx. 21 hours AEL at $25^{\circ} \mathrm{C}$ ) were selected for electrophysiology. All recordings were acquired at $22^{\circ} \mathrm{C}$ from VLM 6 in anterior abdominal segments A2 and A3, in the whole-cell patch clamp configuration essentially as previously described (Broadie and Bate, 1993a). Miniature EJCs were recorded in extracellular HL-3.1 saline (Feng et al., 2004), consisting of (in mM): $\mathrm{NaCl} 70, \mathrm{KCl} 5, \mathrm{MgCl}_{2} 4, \mathrm{NaHCO}_{3}$ 10, trehalose 5, sucrose 115, HEPES 5, $\mathrm{CaCl}_{2}$ 1.5, $\mathrm{pH}$ adjusted to 7.2. Additionally, $2 \mu \mathrm{m}$ TTX was included in the saline to block spontaneous firing of the motoneurons, as the CNS was left attached. The intracellular saline consisted of (in $\mathrm{mM}$ ): $\mathrm{CsCl} 158, \mathrm{ATP}_{\mathrm{NA}} 2$ 2, EGTA 5, Hepes 10, pH adjusted to 7.2 (Yoshihara et al., 2005).

To free the embryo, the egg was dechorionated with commercial bleach. The dissection was carried out in HL-3.1 with $0.5 \mathrm{mM} \mathrm{Ca}^{2+}$ on a coverslip coated with Sylgard (Dow Corning, USA). The head and tail were glued down with histoacryl tissue adhesive glue (Braun, Germany), delivered through a glass pipette (5-10 $\mu \mathrm{m}$ inner diameter), and the incision along the dorsal midline was made with a sharp pipette. The sides were then glued down to remove the internal organs and expose the ventral muscles. Finally, the preparation was enzymatically treated with collagenase (type IV, dissolved to $1 \mathrm{mg} / \mathrm{ml}$ in saline) for 20-25 s to remove the muscle sheath covering VLM 6.

The preparation was viewed in transmitted light with an upright microscope (Olympus, BX51WI) and a 60x water-immersion lens. The patch pipettes were pulled from borosilicate glass $(1.5 \mathrm{~mm}$ outer diameter, with filament) and fire-polished to final resistances of 3-5 M $\Omega$. The seal resistance on the muscle was typically greater than $10 \mathrm{G} \Omega$, and the whole-cell configuration could be achieved with slight suction or an electrical "buzz". The input resistance of the muscle ranged from about $600 \mathrm{M} \Omega$ to $2 \mathrm{G} \Omega$, and the series resistance, measured throughout the experiment, was typically between 10 and $20 \mathrm{M} \Omega$. With maximum currents of $300 \mathrm{pA}$, the series resistance errors (total current $\mathrm{x} \mathrm{R}_{\mathrm{s}}$ ) were deemed acceptable $(<6 \mathrm{mV})$ and were not corrected. The cell membrane capacitance ranged from 17 to $31 \mathrm{pF}$, generating average clamp time constants $\left(\mathrm{R}_{\mathrm{s}} \times \mathrm{C}_{\mathrm{m}}\right)$ of about $380 \mu \mathrm{s}$. 
To record mEJCs, the membrane potential was clamped at $-60 \mathrm{mV}$. A single recording lasted for at least 3 minutes, during which the holding current never exceeded $\pm 25 \mathrm{pA}$. The signals were amplified with an Axopatch 200B (Axon Instruments) patch-clamp amplifier, recorded at a sampling rate of $10 \mathrm{kHz}$ and low-pass filtered at $2 \mathrm{kHz}$. Only events of an amplitude $\geq 20 \mathrm{pA}$ were used for subsequent analysis in pClamp9, Axon Instruments.

\subsubsection{General}

In all experiments that included genotypes expressing GAL4 driven transgenes, the relevant driver was crossed into the wild-type background as a control. Therefore, the controls of separate studies differ slightly in their physiological properties. Except where explicitly listed, control animals in Bruchpilot experiments carried a copy of elav-GAL4.

Unless otherwise noted, the data are reported as mean \pm s.e.m., $n$ indicates the number of cells examined, and $p$ denotes the significance according to the Mann-Whitney Rank Sum test. In the figures, the level of significance is marked with asterisks: ${ }^{*} \mathrm{p} \leq 0.05$; $* * \mathrm{p} \leq 0.01 ; * * * \mathrm{p} \leq 0.001$.

Images of electrophysiological preparations (Fig. 6C and 7) were viewed in transmitted light with an upright microscope (Olympus, BX51WI) fitted with differential interference contrast, and acquired with an Orca-ER (Hamamatsu, Japan) digital camera.

For experiments other than electrophysiology please refer to the methods section of the cited work. 


\section{Results}

\subsection{Bruchpilot}

\subsubsection{The monoclonal antibody Nc82 recognises Bruchpilot}

The monoclonal antibody Nc82 is widely regarded as a reliable neuropil marker in both adult and larval Drosophila (Hofbauer, 1991; Laissue et al., 1999; Wucherpfennig et al., 2003; Rasse et al., 2005; Kittel et al., 2006b; Wagh et al., 2006). In the optic lobe of the adult nervous system, Nc82 binds to terminals of cholinergic (Buchner et al., 1986), GABAergic [ $\gamma$-aminobutyric acid; (Buchner et al., 1988)], and histaminergic synapses (Wagh et al., 2006), and at the larval NMJ, Nc82 staining was found in both glutamatergic and non-glutamatergic synaptic boutons [Fig. 9; (Wagh et al., 2006)]. In type $1 \mathrm{~b}$ boutons Nc82 selectively labelled discrete small spots surrounded by Dynamin, a GTPase of the periactive zone (Fig. 9A). Furthermore, Nc82 exclusively labelled regions exactly opposite, and strictly aligned with the centre of receptor fields (Fig. 9B). These observations indicated that the protein recognised by $\mathrm{Nc} 82$ resides specifically at the presynaptic active zone.
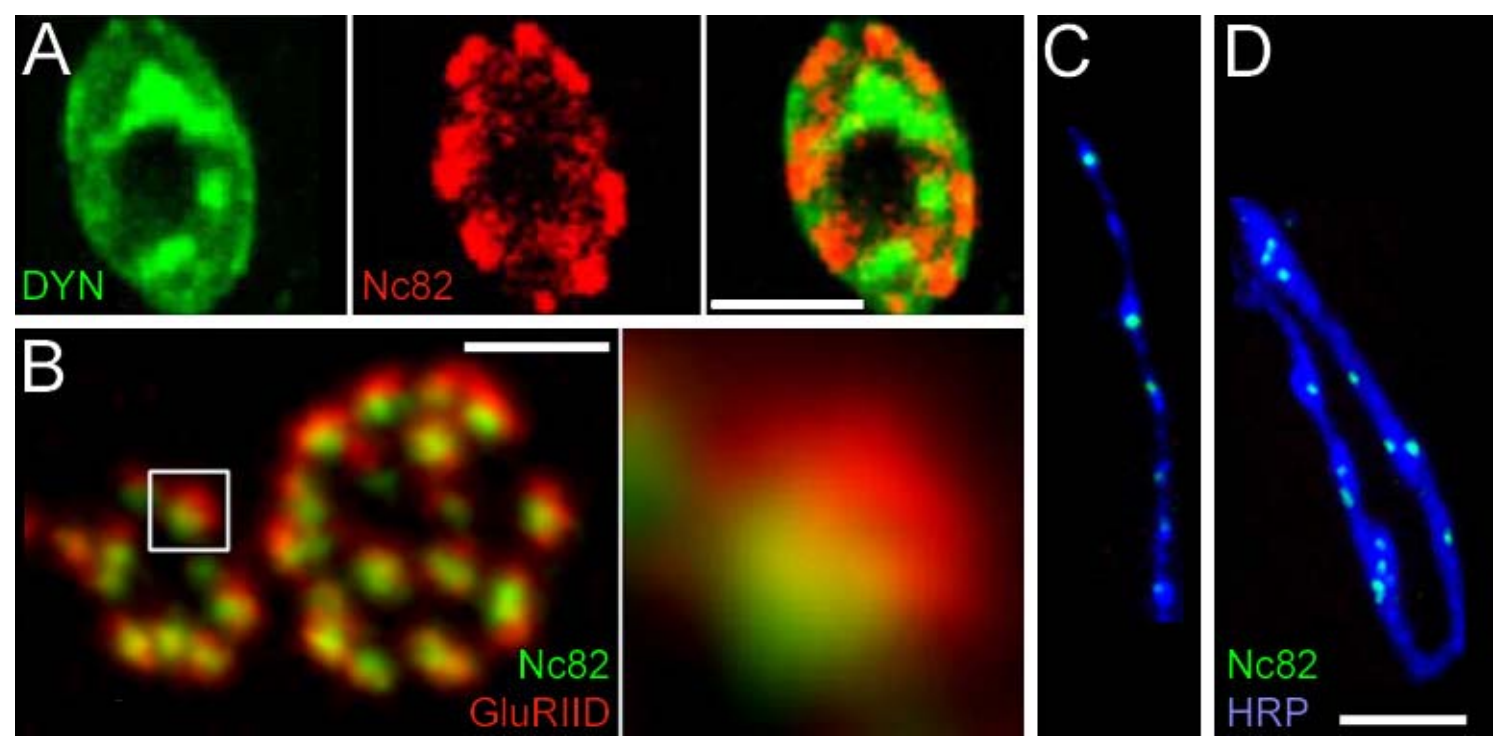

Figure 9. MAB Nc82 labels a component of the presynaptic active zone.

(A, B) Confocal images of type $1 \mathrm{~b}$ synaptic boutons. (A) Nc82 (red) labelled regions not stained by anti-Dynamin [green; (Wagh et al., 2006)]. (B) Nc82 (green) stained the presynaptic area opposite postsynaptic glutamate receptor fields labelled with anti-GluRIID [red; (Kittel et al., 2006b)]. (C, D) Non-glutamatergic type II (C) and type III (D) boutons stained with anti-HRP (blue) and Nc82 [green; (Wagh et al., 2006)]. Scale bars, $2 \mu \mathrm{m}$ in (A, B), $5 \mu \mathrm{m}$ in (C, D). 
Whereas confocal microscopy recognised diffraction-limited spots of Nc82 at neuromuscular active zones (Fig. 10A, left panel), the sub-diffraction resolution of stimulated emission depletion (STED) fluorescence microscopy (Klar et al., 2000; Hell, 2003) revealed donut-shaped structures [Fig. 10A; (Kittel et al., 2006b)]. Viewed perpendicular to the plane of synapses, both single and multiple "rings" were uncovered. The sizes of these rings (Fig. 10B; average length of single rings: $0.191 \pm$ $0.002 \mu \mathrm{m}, \mathrm{n}=204$; average length of single rings within double ring structures: $0.148 \pm$ $0.002 \mu \mathrm{m}, \mathrm{n}=426$; average length of double rings: $0.297 \pm 0.005, \mathrm{n}=213$ ) are in good agreement with previous descriptions of fly active zones, derived from freeze-fracture electron microscopy (Feeney et al., 1998). The donuts were up to $0.16 \mu \mathrm{m}$ high, as judged by images taken parallel to the synaptic plane (Fig. 10A).
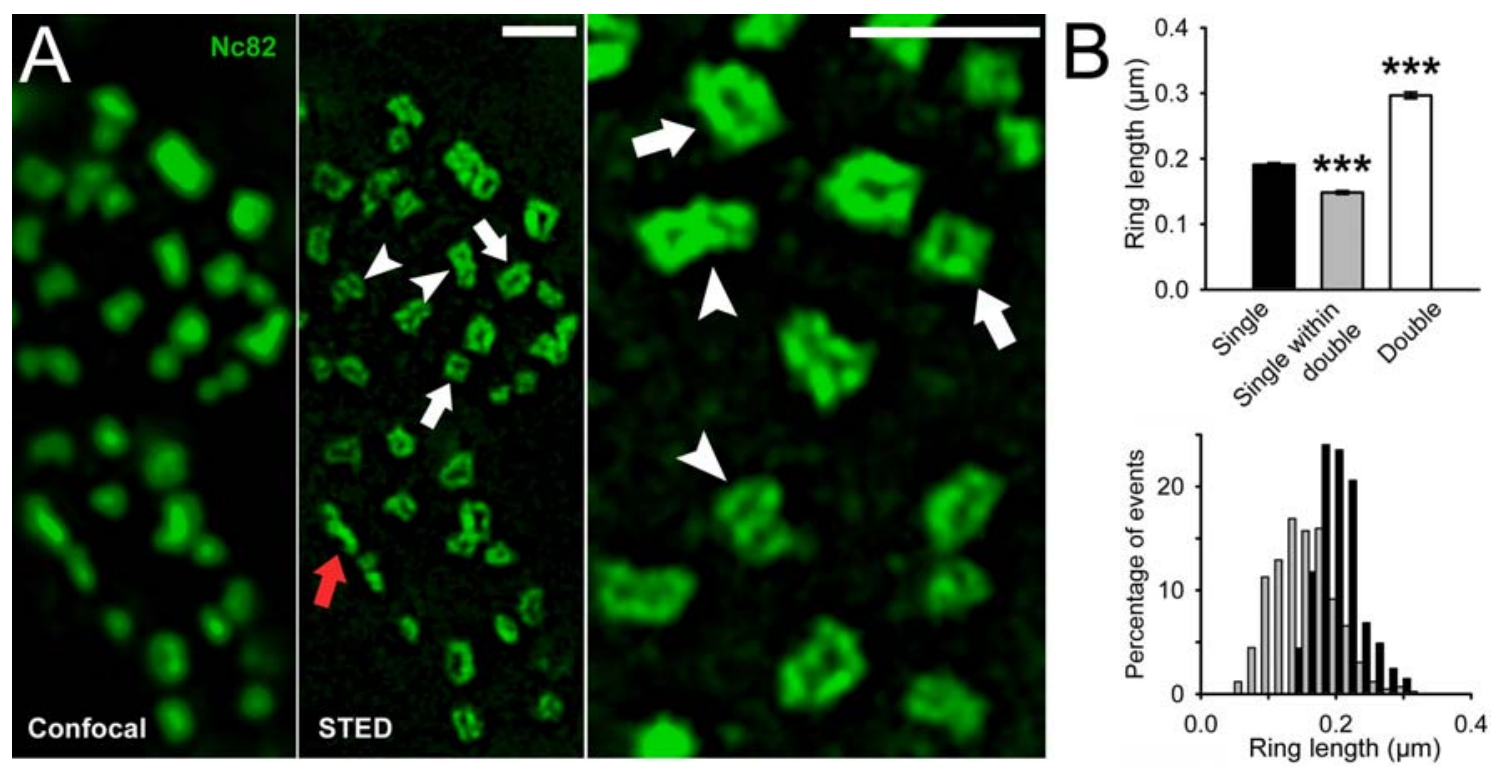

Figure 10. Nc82 recognises donut-shaped structures.

(A) Unlike confocal (left panel), STED microscopy (centre and right panels) revealed donutshaped structures recognised by Nc82 (green) at the NMJ. Viewed from above, both single (white arrows) and clusters of multiple rings (arrowheads) were identified. The red arrow shows a synapse viewed parallel to the synaptic plane. (B) The quantification of the extent of the rings illustrates that individual rings (black) were larger than single rings contained within assemblies of double rings (grey; $\mathrm{p}<0.001$ ). Scale bars, $250 \mathrm{~nm}$ (Kittel et al., 2006b).

A previously unknown protein of roughly $200 \mathrm{kDa}$ was identified as the Nc82 antigen and a novel component of Drosophila active zones (Wagh et al., 2006). Subsequently named Bruchpilot (BRP; from German 'Crashpilot'; Owing to a behavioural defect of 
an 'unfortunate' hypomorphic line), this protein is encoded by a large complex locus (Fig. 11A), and is apparently only expressed in postmitotic differentiated neurons. The Nc82 epitope was mapped to the C-terminal 1105 amino acids of the 1740 amino acid protein, which is characterised by coiled-coil domains over its entire length [Fig. 11; (Wagh et al., 2006)]. The N-terminus of Bruchpilot exhibits a significant homology to the mammalian ELKS/CAST (Ohtsuka et al., 2002)/ERC [ELKS/Rab6-interacting protein/CAST; (Wang et al., 2002)] proteins, which are associated with the cytomatrix at the active zone (Fig. 11B). But BRP neither contains the PDZ-interaction motif (IWA) for RIM, as found in mammalian and C. elegans ELKS proteins, nor could any direct vertebrate homologue be detected for the large C-terminus of BRP. However, the C-terminal half of BRP shows a significant similarity to a number of large cytoskeletal proteins like plectin and myosin heavy chain (Wagh et al., 2006).

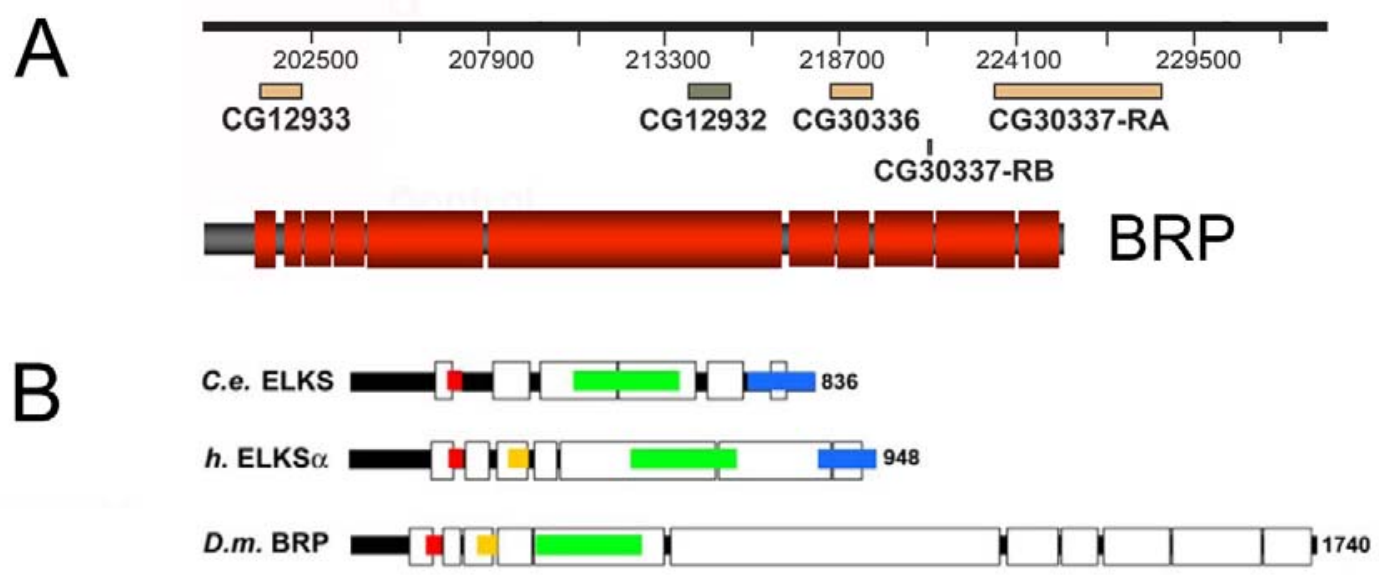

Figure 11. Drosophila Bruchpilot shows N-terminal homology to ELKS/CAST/ERK.

(A) The brp locus consists of 18 exons (organized in 3 exon-clusters), which were previously annotated as three independent genes (CG12933, CG30336 and CG30337). The BRP protein is rich in coiled-coil domains (red boxes). (B) Comparisons of predicted coiled-coil domains (white boxes) and conserved regions (colour) for C. elegans, human, and Drosophila homologues. The N-terminal 480 amino acids of Bruchpilot contain short homologous stretches of up to $67 \%$ identity with both mammalian and C. elegans ELKS/CAST/ERC (red, yellow, and green), but Bruchpilot lacks the IWA motif [blue; (Wagh et al., 2006)]. 


\subsubsection{Synaptic function following suppression of Bruchpilot}

As a first investigation into the involvement of BRP in synaptic structure and function, transgene-mediated RNA interference (RNAi) was used in combination with the GAL4/UAS system (Brand and Perrimon, 1993) to suppress BRP expression in a tissueand time-specific manner. In particularly, the effect of a UAS-controlled construct was analysed, that encoded snap-back, double stranded RNA corresponding to CG30337 (C8), and was driven panneuronally with elav-GAL4 [RNAi ${ }^{\mathrm{C}}$; (Wagh et al., 2006)].
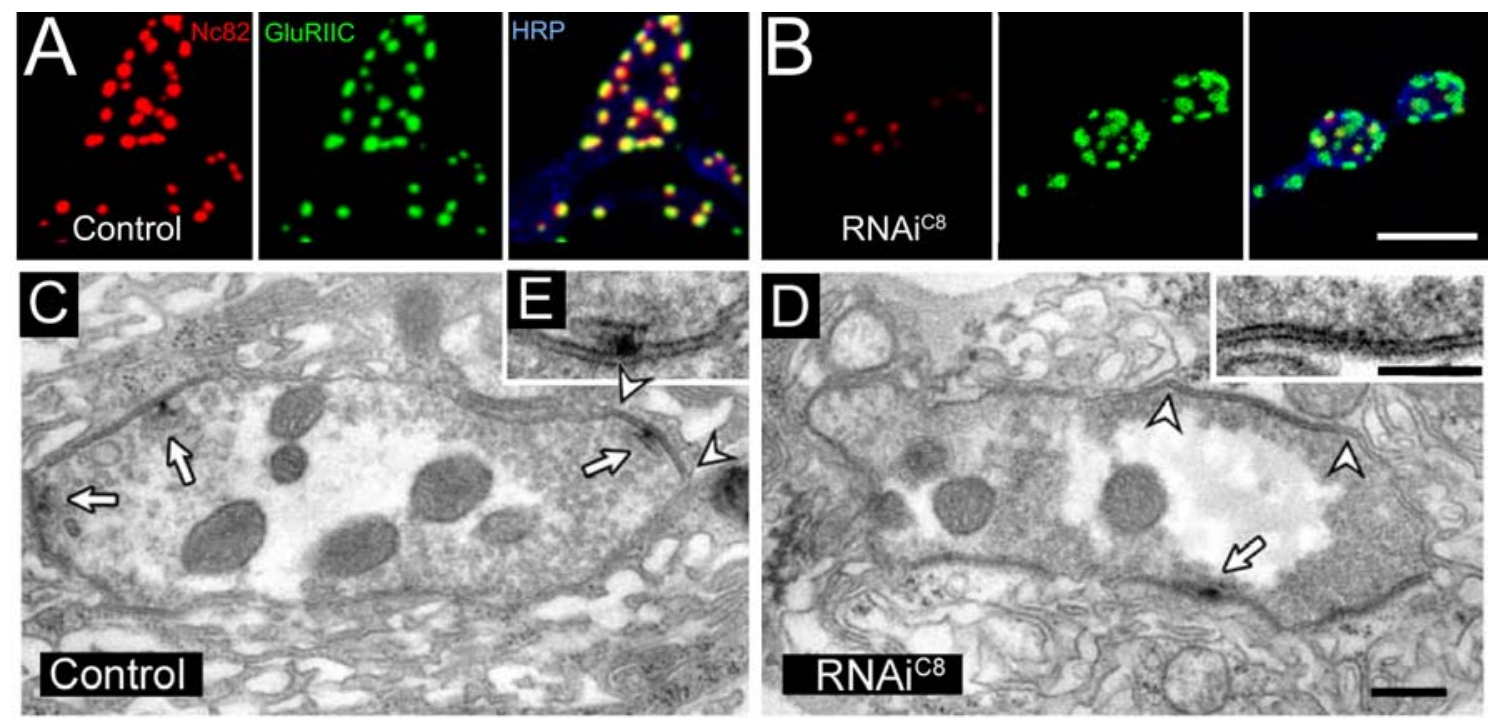

Figure 12. Defective active zone structure following suppression of Bruchpilot.

(A, B) Triple staining of control (A) and $\mathrm{RNAi}^{\mathrm{C} 8}$ (B) larval NMJs with anti-BRP (Nc82; red), anti-GluRIIC (green), and anti-HRP (blue), showed the strongly reduced BRP signal at RNAi ${ }^{\text {C8 }}$ synapses. (C-E) Transmission electron micrographs of type 1 b boutons. (C) Control synapses (example marked by arrowheads) often displayed presynaptic T-bars (arrow), whereas (D) $\mathrm{RNAi}^{\mathrm{C} 8}$ synapses were only rarely decorated with T-bars. Representative synapses are shown in the insets for control (E) and RNAi ${ }^{\mathrm{C} 8}$ larvae. Scale bars, $5 \mu \mathrm{m}$ in (A, B) and $250 \mathrm{~nm}$ in $[\mathrm{C}, \mathrm{D}, \mathrm{E}$; (Wagh et al., 2006)].

At neuromuscular junctions of $\mathrm{RNAi}^{\mathrm{C} 8}$ larvae the $\mathrm{Nc} 82$ signal was clearly reduced (Fig. 12A). Despite an overall normal junctional morphology [no obvious change in the size of the NMJ, receptor fields, number of boutons, and number of receptor fields (Control: $540 \pm 166$ receptor fields; $\mathrm{RNAi}^{\mathrm{C} 8}: 462 \pm 166$ receptor fields; $\mathrm{n}=9$ NMJs each; $\mathrm{p}>0.05$, t-test)], the ultrastructural integrity of presynaptic active zones was impaired (Fig. 12C-E). Roughly $40 \%$ of control synapses displayed electron-dense T-bars (25 T- 
bars at 61 synapses in 21 sections), and though the density of synapses was similar at $\mathrm{RNAi}^{\mathrm{C} 8} \mathrm{NMJ}$, only $7 \%$ of these were associated with T-bars (8 T-bars at 108 synapses in 35 sections). When $\mathrm{RNAi}^{\mathrm{C} 8}$ was selectively driven in the adult visual system ( $\mathrm{gmr}$ Gal4 driver) a complete elimination of T-bars was noted in photoreceptor terminals (Wagh et al., 2006).

To investigate the physiological effects of BRP suppression, two-electrode voltage clamp recordings were acquired from the larval NMJ (Wagh et al., 2006). Both the average amplitude and frequency of minis at $\mathrm{RNAi}^{\mathrm{C} 8} \mathrm{NMJs}$ were indistinguishable from those of controls (Fig. 13A; Mini amplitude: Control: $-0.86 \pm 0.04 \mathrm{nA}, \mathrm{n}=10$; $\mathrm{RNAi}^{\mathrm{C}}$ : $-0.93 \pm 0.05 \mathrm{nA}, \mathrm{n}=12 ; \mathrm{p}=0.323$; Mini frequency: Control: $0.63 \pm 0.07 \mathrm{~Hz}, \mathrm{n}=9$; $\mathrm{RNAi}^{\mathrm{C} 8}: 0.63 \pm 0.09 \mathrm{~Hz}, \mathrm{n}=12 ; \mathrm{p}=0.972$ ). Thus, the reduction, but not complete elimination, of BRP levels did not appear to influence the response of individual postsynaptic receptor fields to transmitter release.
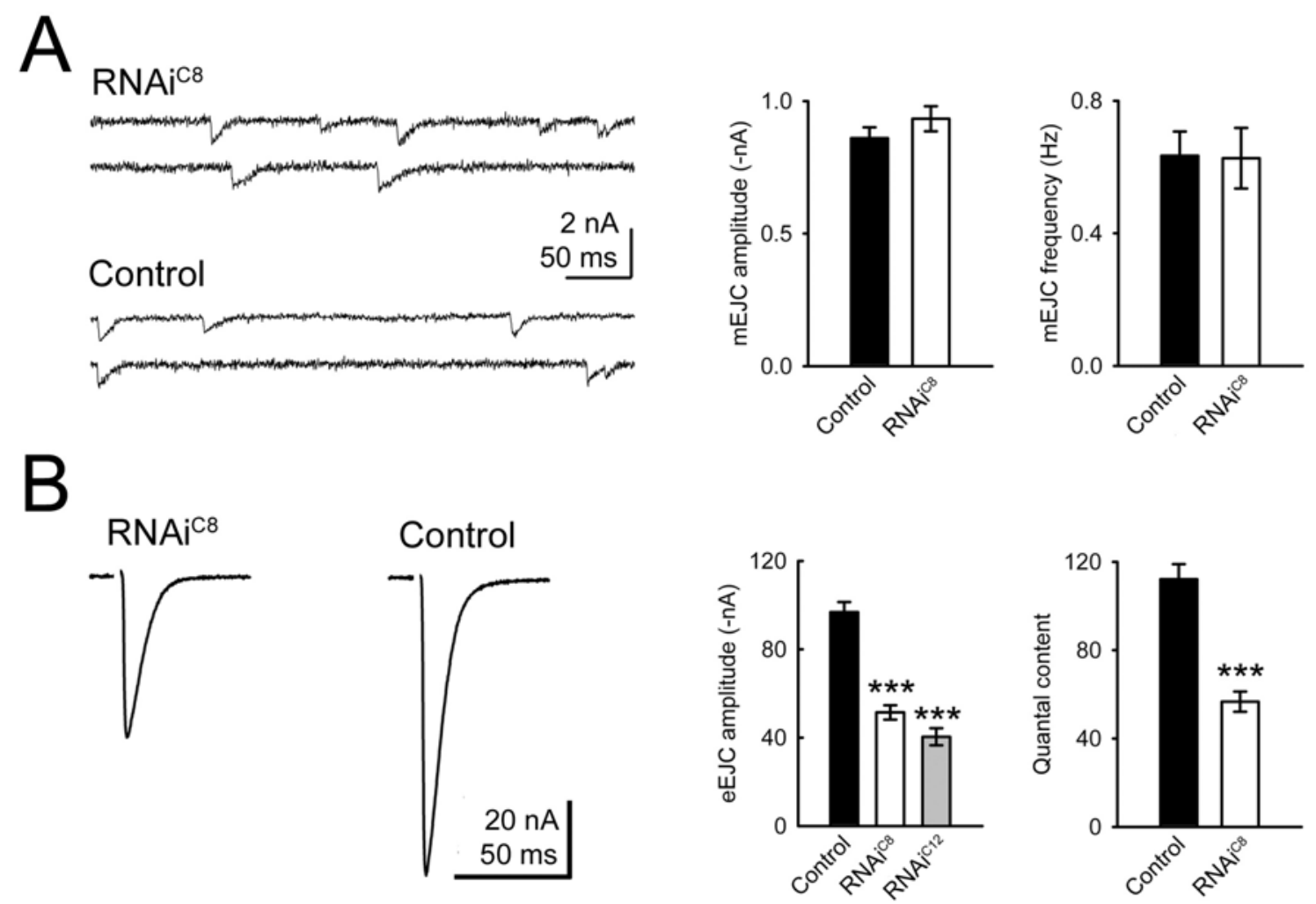

Figure 13. Suppresion of Bruchpilot impairs neurotransmitter release.

(A) Amplitude and frequency of mEJCs were unaltered at RNAi ${ }^{\mathrm{C} 8} \mathrm{NMJs}$. (B) As the amplitude of eEJCs $\left(0.2 \mathrm{~Hz}\right.$ stimulation frequency) was significantly reduced in $\mathrm{RNAi}^{\mathrm{C} 8}$ larvae, it follows that the number of vesicles released per action potential (quantal content) was decreased following BRP suppression. A second RNAi line $\left(\mathrm{RNAi}^{\mathrm{C} 12}\right)$ was used to confirm the reduced eEJC amplitude (Wagh et al., 2006). 
In contrast, the average amplitude of eEJCs at a low stimulation frequency was reduced to about $50 \%$ of wild-type levels following BRP suppression (Control: $-96.8 \pm 4.6 \mathrm{nA}$, $\mathrm{n}=11 ;$ RNAi $^{\mathrm{C} 8}:-51.4 \pm 3.2 \mathrm{nA}, \mathrm{n}=12, \mathrm{p}<0.001 ; \mathrm{RNAi}^{\mathrm{C} 12}:-40.4 \mathrm{nA} \pm 3.9 \mathrm{nA}, \mathrm{n}=8$; $\mathrm{p}<0.001)$. Considering the unaltered amplitude of mEJCs, it followed that the quantal

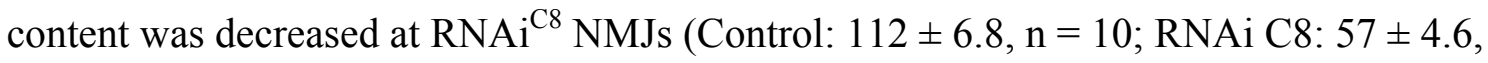
$\mathrm{n}=12 ; \mathrm{p}<0.001)$. Furthermore, as the number of synapses was sustained following BRP suppression, it was concluded that the neurotransmitter release defect could either be attributed to a diminished synaptic vesicle release probability, a decrease in the number of vesicles available for release, or a reduction in the number of functionally intact synapses (Wagh et al., 2006). 


\subsubsection{Synaptic function following complete elimination of Bruchpilot}

To address these considerations, we sought to eliminate BRP function completely. By employing transposon-mediated mutagenesis, a mutant chromosome (brp ${ }^{69}$ ) was isolated, that lacked nearly the entire open reading frame of Bruchpilot [Fig. 14A; (Kittel et al., 2006b)]. brp mutants (brp $\left.{ }^{69} / d f(2 R) B S C 29\right)$ still developed into mature larvae, but could not form pupae. As expected, the Nc82 label was completely lost from synapses of brp mutant NMJs, but could be restored by re-expressing the brp cDNA in the mutant background with the neuron-specific driver lines ok6-GAL4 (Fig. 14B) or elav-GAL4. This also rescued larval lethality, giving pupae.
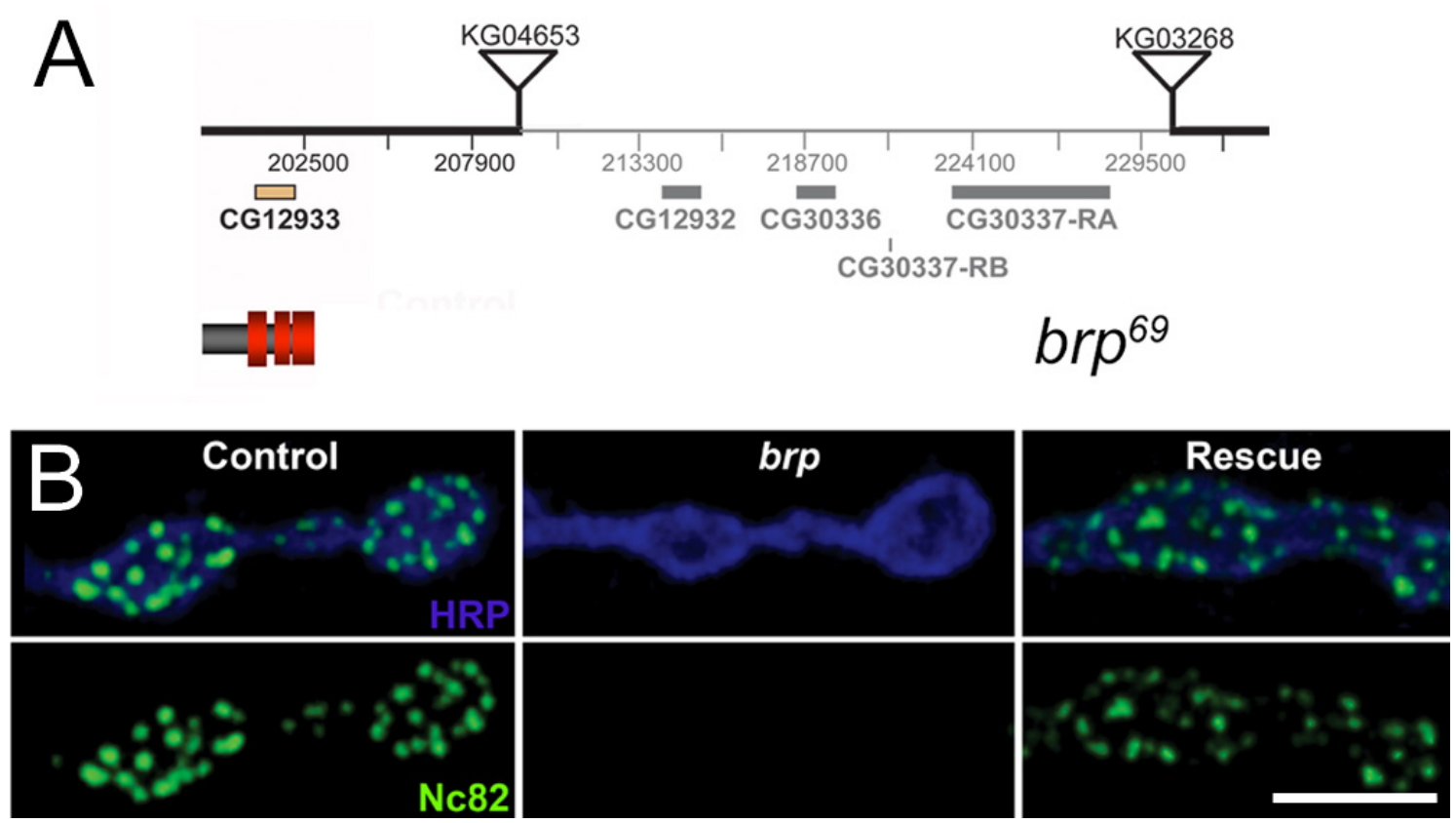

Figure 14. Mutants of bruchpilot lack the neuromuscular Nc82 label.

(A) In the $b r p^{69}$ allele the DNA between the two indicated P-transposons (triangles) downstream of the locus CG12933 was deleted. Thus, most of the BRP protein (red boxes: coiled-coil domains) could no longer be formed. (B) The Nc82 label (green) was entirely lost from brp mutant neuromuscular synapses (labelled by anti-HRP in blue), but could be restored by reexpressing the brp cDNA with ok6-GAL4 (rescue). Scale bar, $4 \mu \mathrm{m}$ (Kittel et al., 2006b).

Mutants had slightly smaller NMJs (Control: $780.0 \pm 35.8 \mu \mathrm{m}^{2}, \mathrm{n}=14$; brp: $593.3 \pm$ $\left.29.1 \mu \mathrm{m}^{2}, \mathrm{n}=12 ; \mathrm{p}=0.0013\right)$, as determined by the projected surface area of anti-HRP staining (Fig. 15A), and accordingly, brp mutant NMJs also had fewer individual synapses (Control: $411.1 \pm 41.5, \mathrm{n}=9$ NMJs; brp: $296.3 \pm 28.9, \mathrm{n}=8$ NMJs; $\mathrm{p}=0.036$ ), as judged by the presence of the glutamate receptor subunit GluRIID. 
However, individual receptor fields were enlarged in mutants (Fig. 15B; Control: $0.43 \pm$ $0.02 \mu \mathrm{m}^{2}, \mathrm{n}=9$ NMJs; brp: $0.64 \pm 0.03 \mu \mathrm{m}^{2}, \mathrm{n}=8$ NMJs; $\left.\mathrm{p}<0.001\right)$. Thus, principal synapse formation proceeded normally in brp mutants, with individual postsynaptic receptor fields increased in size but moderately decreased in number, such that the overall surface area covered by receptor fields was comparable between the two genotypes (Control: $180.6 \pm 26.8 \mu \mathrm{m}^{2}, \mathrm{n}=9$ NMJs; brp: $191.1 \pm 22.9 \mu \mathrm{m}^{2}, \mathrm{n}=8$ NMJs; $\mathrm{p}=0.67)$.

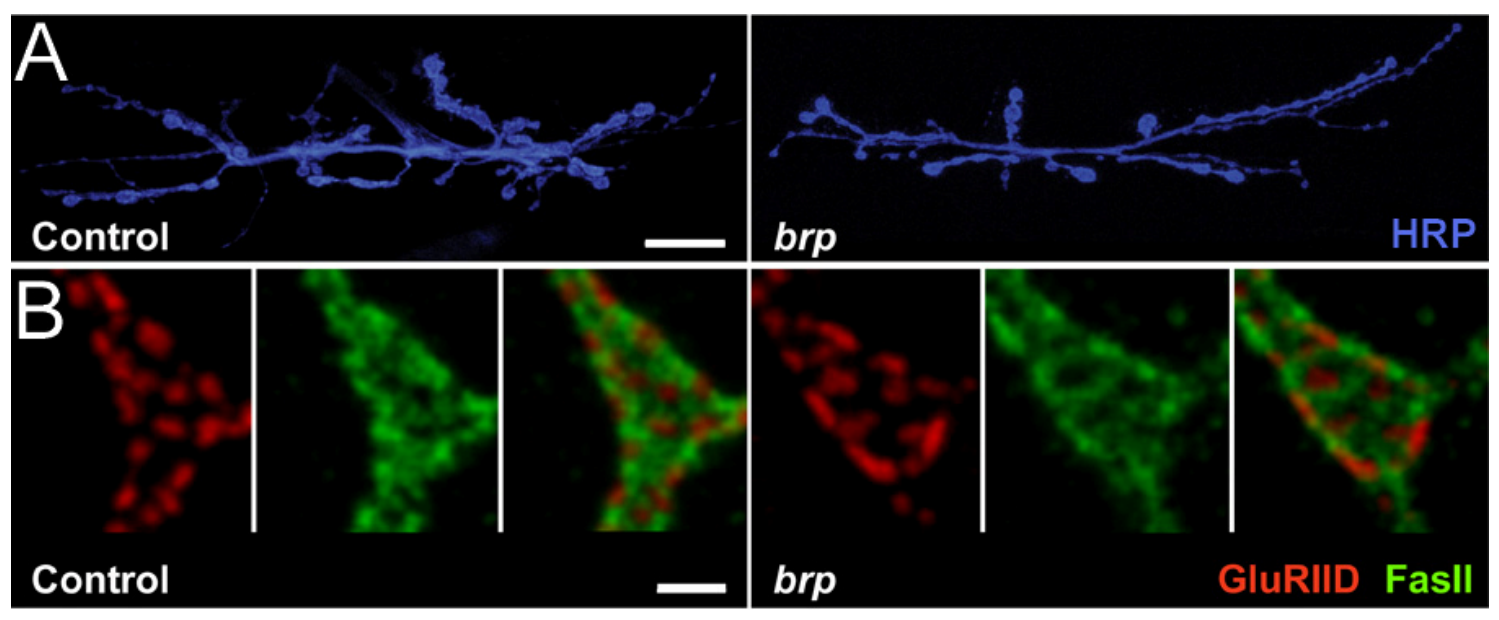

Figure 15. Junctional morphology of bruchpilot mutants.

(A) Staining with anti-HRP (blue) demonstrated the normal morphological organisation of brp mutant NMJs. (B) Receptor fields (stained with anti-GluRIID, red) were surrounded by the characteristic perisynaptic expression of the NCAM homologue Fas II (green) in both controls and mutants. Scale bars, $20 \mu \mathrm{m}$ in (A) and $2 \mu \mathrm{m}$ in [B; (Kittel et al., 2006b)].

Next, electron microscopy was used to study the synaptic structure in more detail [Fig. 16; (Kittel et al., 2006b)]. At brp mutant NMJs, synapses were present at regular density and consistent with the enlarged glutamate receptor fields (Fig. 15B) postsynaptic densities appeared larger while structurally intact (Fig. 16C, D). However, intermittent rufflings or invaginations of the presynaptic membrane were noted (Fig. 16D) and brp mutant synapses completely lacked T-bars. Residual electron-dense material attached to the presynaptic active zone membrane was only rarely identified (Fig. 16 E; Control: 56 sections, 216 synapses, 74 T-bars, $\mathrm{n}=3$ NMJs; brp: 37 sections, 164 synapses, no T-bars and 8 cases of residual material, $\mathrm{n}=4 \mathrm{NMJs}$ ). After reexpressing the Brp protein in the mutant background, T-bar formation could be partially restored (Fig. 16E; 40 sections, 129 synapses, 20 T-bars, $\mathrm{n}=2$ NMJs), though these 
structures were occasionally somewhat aberrant in shape. Thus, Brp assists in the ultrastrucutral assembly of the active zone, and is essential for T-bar formation. This conclusion is supported by the data obtained from $\mathrm{RNAi}^{\mathrm{C} 8} \mathrm{NMJs}$, as the severity of the structural phenotype correlated with the BRP expression level.
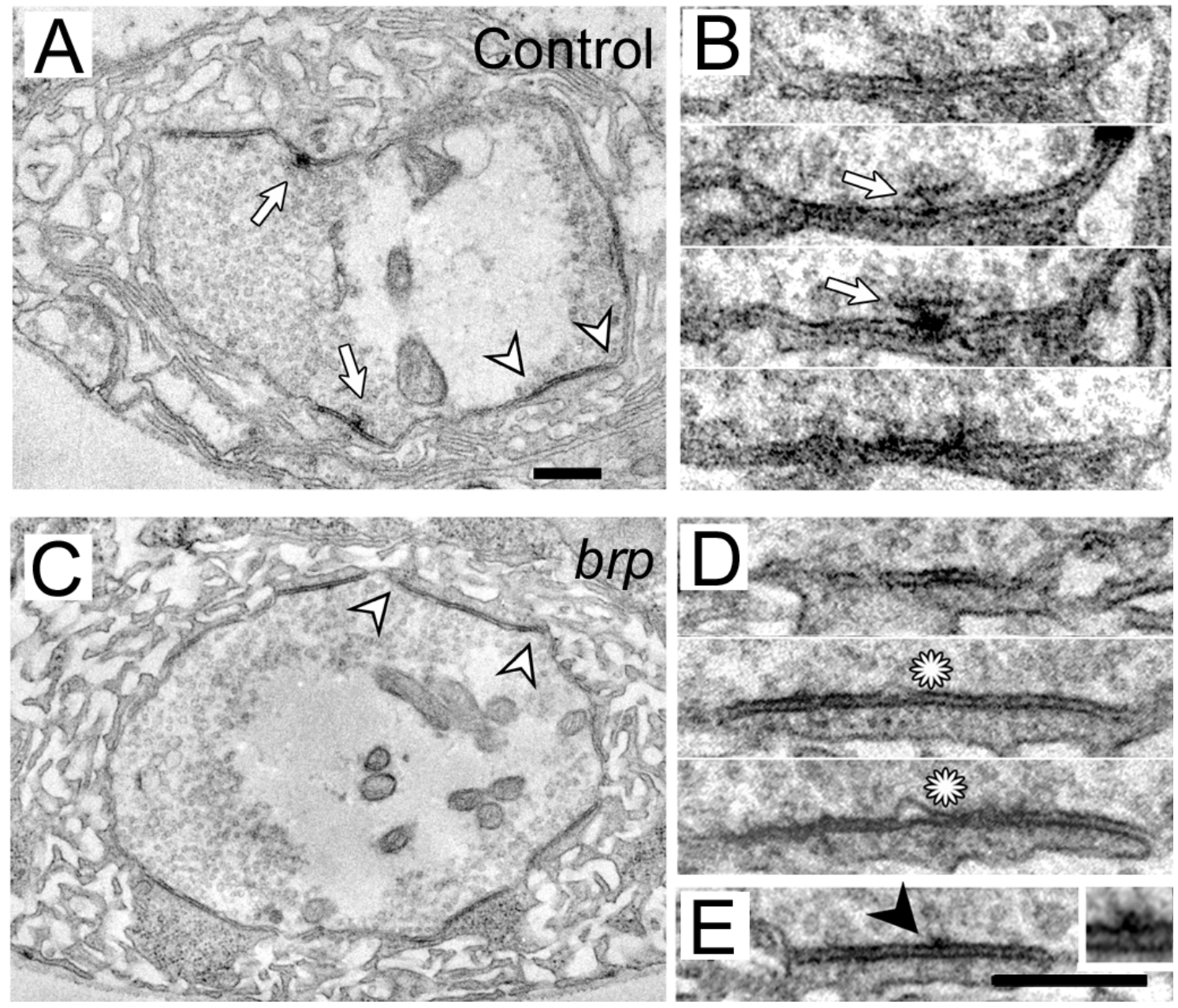

Figure 16. Complete loss of T-bars from bruchpilot mutant neuromuscular synapses.

(A) Electron micrograph of a control type 1b synaptic bouton displaying synapses (arrowheads) frequently endowed with T-bars (arrows). (B) $85-90 \mathrm{~nm}$ thick serial sections of control synapses. A T-bar was observed in two consecutive sections (arrows). (C) Overall normal organisation of a brp mutant bouton with synapses identified by closely apposed electron-dense pre- and postsynaptic membranes (arrowheads), but without T-bars. (D) Serial sections of a representative brp mutant synapse with presynaptic membrane rufflings (asterisks). (E) Residual electron-dense material was occasionally observed at brp mutant active zone membranes (arrowhead). Scale bars, $250 \mathrm{~nm}$ (Kittel et al., 2006b).

To analyse the physiological consequences following a complete loss of BRP, TEVC recordings were made from brp mutant larval NMJs [Fig. 17; (Kittel et al., 2006b)]. At 
low stimulation frequencies we noted a drastic decrease in eEJC amplitudes (Fig. 17A; elav-GAL4 background Control: $-89.3 \pm 3.4 \mathrm{nA}$; brp: $-32.1 \pm 5.9 \mathrm{nA}, \mathrm{n}=10$ each; $\mathrm{p}<0.001$; ok6-GAL4 background Control: $-89.6 \pm 4.4 \mathrm{nA}, \mathrm{n}=9$; brp: $-32.8 \pm 3.7 \mathrm{nA}$, $\mathrm{n}=10 ; \mathrm{p}<0.001)$. This drop in current amplitude could be partially rescued through brp re-expression within the presynaptic motoneurons using either elav-GAL4 or ok6GAL4 (elav-GAL4: $-55.5 \pm 4.3 \mathrm{nA}, \mathrm{n}=11 ; \mathrm{p}=0.01$ to brp; ok6-GAL4: $-62.2 \pm 5.3 \mathrm{nA}$, $\mathrm{n}=10 ; \mathrm{p}=0.002$ to $\mathrm{brp}$ ). In contrast, the amplitude of mEJCs in response to single, spontaneous vesicle fusion events was increased over control levels (Fig. 17B; Control: $-0.84 \pm 0.06 \mathrm{nA}$; brp: $-1.17 \pm 0.05 \mathrm{nA} ; \mathrm{n}=10$ each; $\mathrm{p}=0.004)$. This is consistent with the enlarged individual glutamate receptor fields of brp mutants (Fig. 15B) and excludes a lack of postsynaptic sensitivity as the cause of the reduced nerve-evoked current amplitudes.
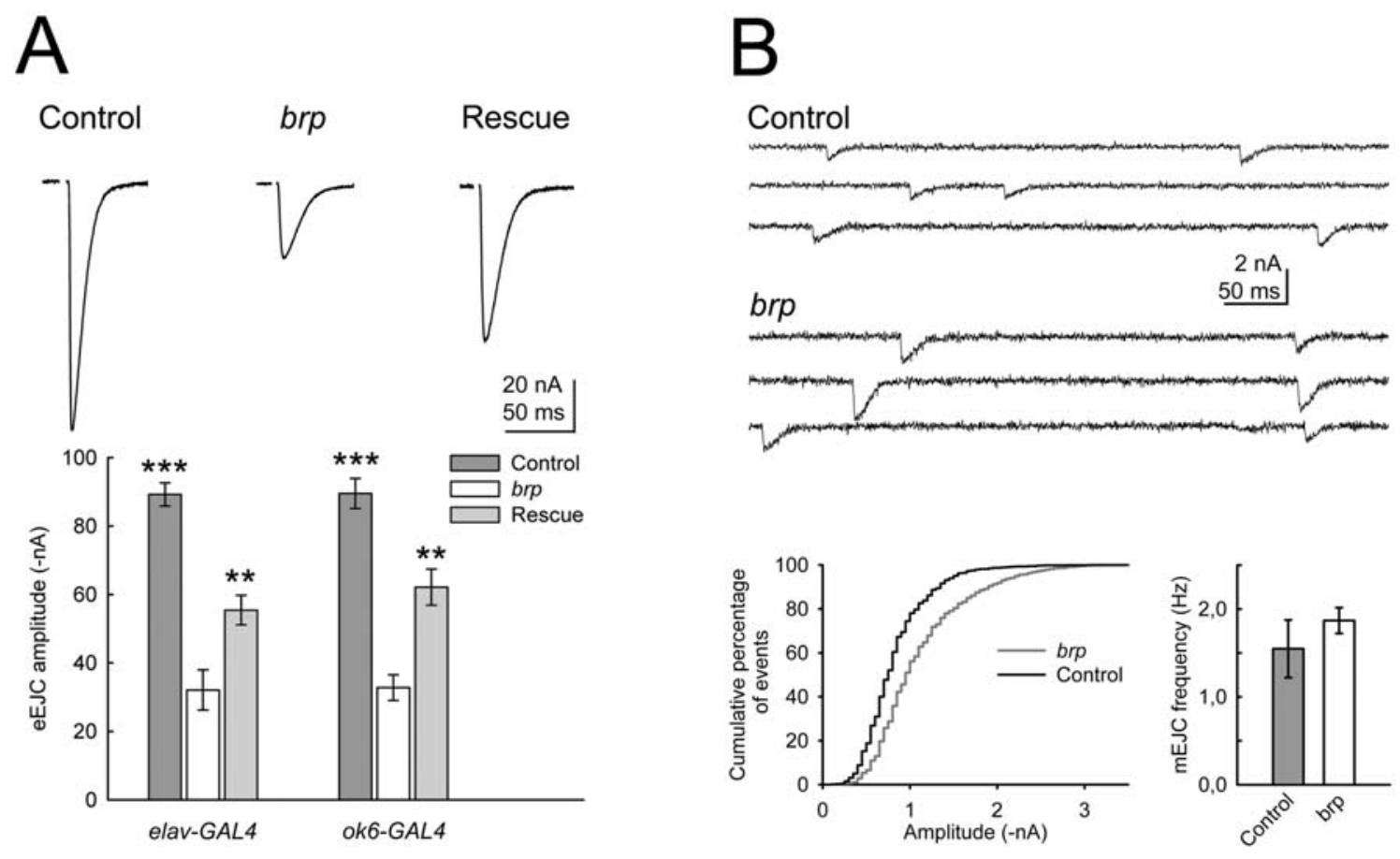

Figure 17. Electrophysiological characterisation of bruchpilot mutant NMJs.

(A) Average traces of eEJCs at $0.2 \mathrm{~Hz}$ nerve stimulation (top) and mean eEJC amplitudes of control (dark grey), brp mutant (white), and rescued animals (light grey), carrying either a copy of elav-GAL4 or ok6-GAL4. (B) Sample traces of mEJCs and a cumulative histogram of the amplitude distribution (0.05 nA bins). The average mEJC amplitude was increased in brp mutants, whereas the frequency was not significantly altered. Thus the quantal content of brp mutant NMJs was significantly reduced with respect to controls (Kittel et al., 2006b). 
Given the decrease of evoked and increase of miniature EJC amplitudes, it followed that the quantal content was severely compromised at brp mutant NMJs (Control: $109 \pm 5.7$; brp: $28 \pm 5.2 ; \mathrm{n}=10$ each; $\mathrm{p}<0.001)$ to an extent that could not be solely attributed to a moderate decrease in the number of synapse (see above). Considering the ultrastructural defects, it was conceivable that brp mutant synapses suffered from an inadequate targeting of vesicles to the active zone membrane, thereby impairing exocytosis. The number of vesicles directly docked to active zone membranes was slightly decreased in brp mutants (Control: $1.10 \pm 0.13$ per active zone, 51 synapses, $\mathrm{n}=3 \mathrm{NMJs}$; brp: $0.87 \pm$ 0.09 per active zone, 89 synapses, $\mathrm{n}=4 \mathrm{NMJs} ; \mathrm{p}=0.53$ ). However, the amplitude distribution and sustained frequency of mEJCs (Fig. 17B; Control: $1.55 \pm 0.33 \mathrm{~Hz}$; brp: $1.87 \pm 0.15 \mathrm{~Hz} ; \mathrm{n}=10$ each; $\mathrm{p}=0.186$ ) illustrated that brp mutant synapses did not appear to suffer from 'extrasynaptic release', as would be caused by a misalignment of vesicle fusion sites with postsynaptic receptors. And appropriately, both exo- and endocytotic proteins appeared distributed normally at brp mutant synapses (Fig. 18).
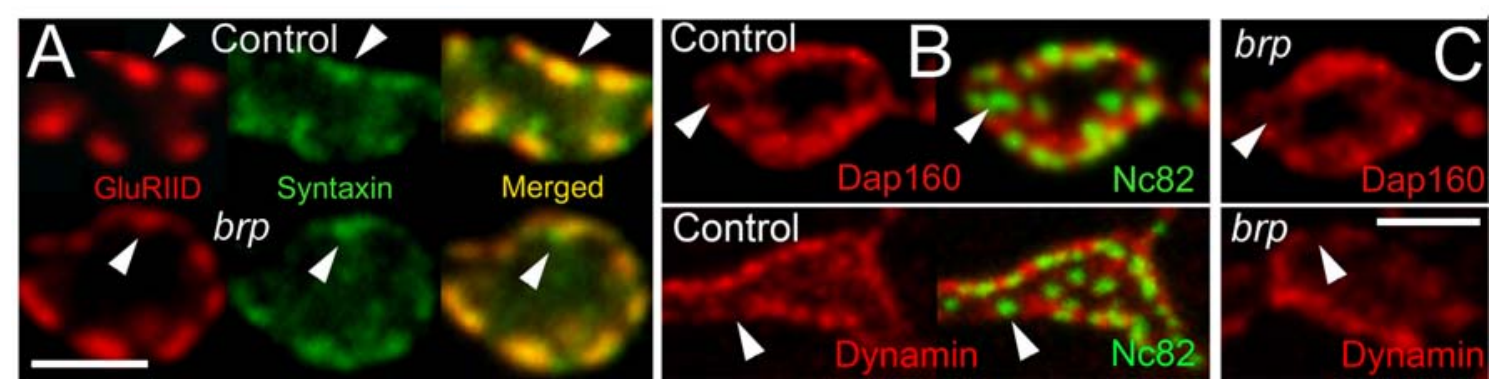

Figure 18. Distribution of exo- and endocytotic proteins at bruchpilot mutant NMJs.

(A) The exocytotic protein Syntaxin co-localized with GluRIID (arrowheads) at both control and brp mutant synapses. (B) The endocytotic proteins Dap160 and Dynamin surrounded the Nc82 labelled active zones in controls (arrowheads). (C) At brp mutant synapses a similar distribution of these markers was observed (arrowheads). Scale bars: $2 \mu \mathrm{m}$ (Kittel et al., 2006b).

In view of the structural requirement for Bruchpilot at the active zone, and the association of this specialised compartment with $\mathrm{Ca}^{2+}$ channels, it was reasoned that aberrant $\mathrm{Ca}^{2+}$ signalling may underlie the release defect of brp mutant synapses. The exact amplitude and time course of action potential-triggered $\mathrm{Ca}^{2+}$ influx in the nerve terminal governs the amplitude and time course of vesicle release (Barrett and Stevens, 1972). Upon inspecting the temporal profile of postsynaptic currents (Fig. 19) it was observed that nerve-evoked responses of brp mutants were delayed (Rise time: $2.53 \pm$ 
$0.37 \mathrm{~ms} ; \mathrm{n}=10$ ) when compared with controls (Rise time: $1.11 \pm 0.05 \mathrm{~ms} ; \mathrm{n}=10 ; \mathrm{p}<$ 0.001 ), whereas in contrast $\mathrm{mEJC}$ rise times were unchanged (Control: $1.06 \pm 0.04 \mathrm{~ms}$; brp: $1.06 \pm 0.03 \mathrm{~ms} ; \mathrm{n}=10$ each). Thus, evoked vesicle fusion events appeared less synchronised with the invasion of the presynaptic terminal by an action potential (Kittel et al., 2006b).
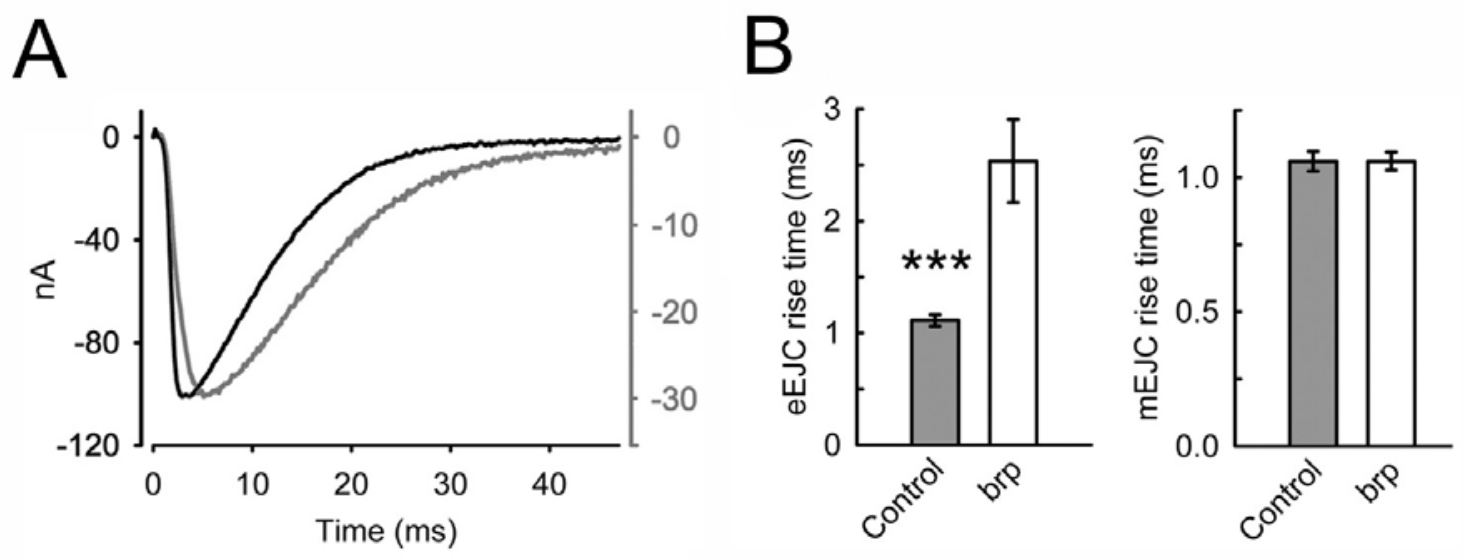

Figure 19. Altered kinetic properties of evoked responses in bruchpilot mutants.

(A) Average scaled eEJCs (Control $=$ black, $b r p=$ grey) illustrate the delayed release in brp mutants compared to controls. (B) Whereas the rise time of eEJCs was significantly increased at brp NMJs, the rise time of mEJCs was indistinguishable from the control (Kittel et al., 2006b).

Spatio-temporal changes in $\mathrm{Ca}^{2+}$ influx have a profound effect on short-term plasticity (Atwood, 1967; Rozov et al., 2001; Zucker and Regehr, 2002). Thus, higher frequency stimulation was applied to the NMJ (Kittel et al., 2006b). Whereas at $10 \mathrm{~Hz}$ controls $(n=18)$ exhibited a substantial short-term depression of eEJC amplitudes, brp mutants $(n=15)$ showed strong initial facilitation before stabilising at a slightly lower, but frequency-independent steady-state current (Fig. 20A; Control at $10 \mathrm{~Hz}:-54.7 \pm 3.3 \mathrm{nA}$; brp: $-35.6 \pm 3.0 \mathrm{nA} ; \mathrm{p}<0.001)$. Two conclusions could be drawn from this response to tetanic stimulation. First, as judged by the initial facilitation at $10 \mathrm{~Hz}$, neither a reduction in the number of releasable vesicles, nor available release sites could fully account for the low quantal content of brp mutants at moderate stimulation frequencies. And second, the altered short-term plasticity of brp mutant synapses suggested a change in the highly $\mathrm{Ca}^{2+}$-dependent vesicle release probability (Dittman et al., 2000). To investigate this in more detail, paired-pulse protocols were used (Fig. 20B). Closely spaced stimuli lead to a build-up of residual $\mathrm{Ca}^{2+}$ in the vicinity of presynaptic $\mathrm{Ca}^{2+}$ 
channels, thereby enhancing the probability of a vesicle within this local $\mathrm{Ca}^{2+}$ domain to undergo fusion following the next pulse (Katz and Miledi, 1968). The absence of marked facilitation at control synapses (Ratio at $30 \mathrm{~ms}$ interval: $1.1 \pm 0.03$ ) could be explained by a depletion of release-ready vesicles (von Gersdorff et al., 1997). At brp mutant NMJs, however, the prominent facilitation at short inter-pulse intervals (Ratio at $30 \mathrm{~ms}$ interval: $2.0 \pm 0.13 ; \mathrm{p}<0.001)$ illustrated that the enhancement of release probability strongly outweighed the depletion of releasable vesicles. Thus, initial vesicle release probability was low, and release at brp mutant synapses particularly benefited from the accumulation of intracellular $\mathrm{Ca}^{2+}$ (Kittel et al., 2006b).
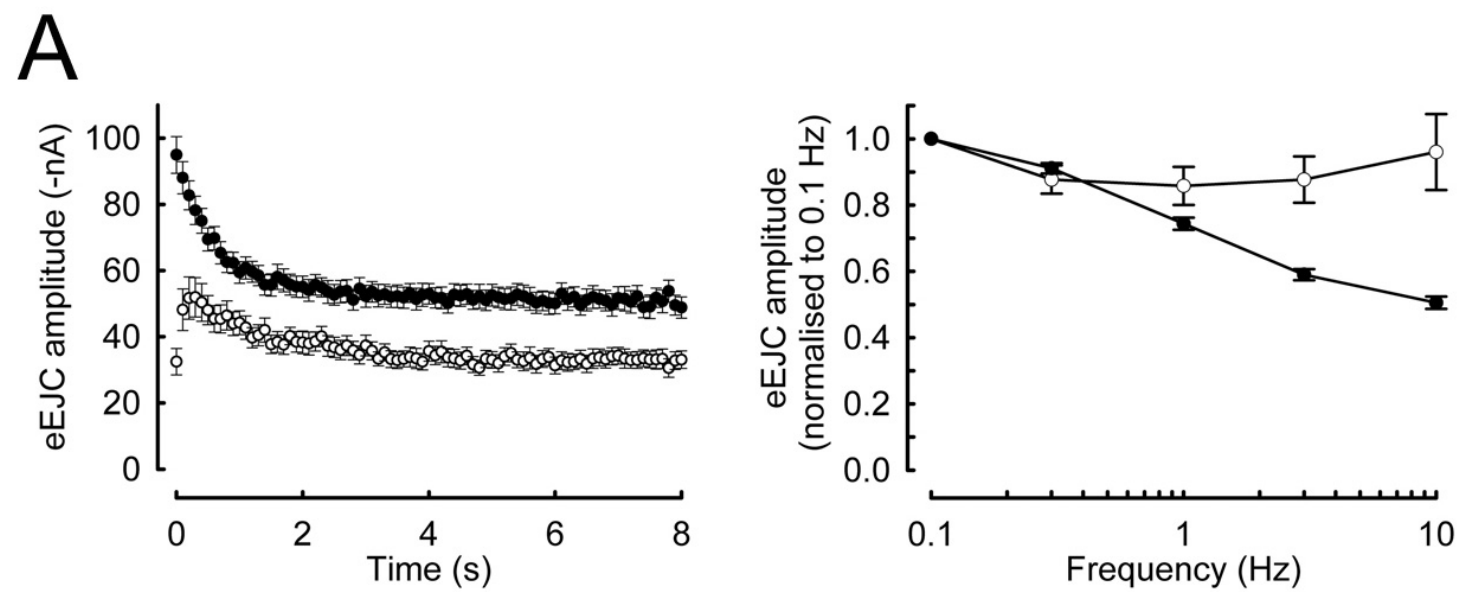

B
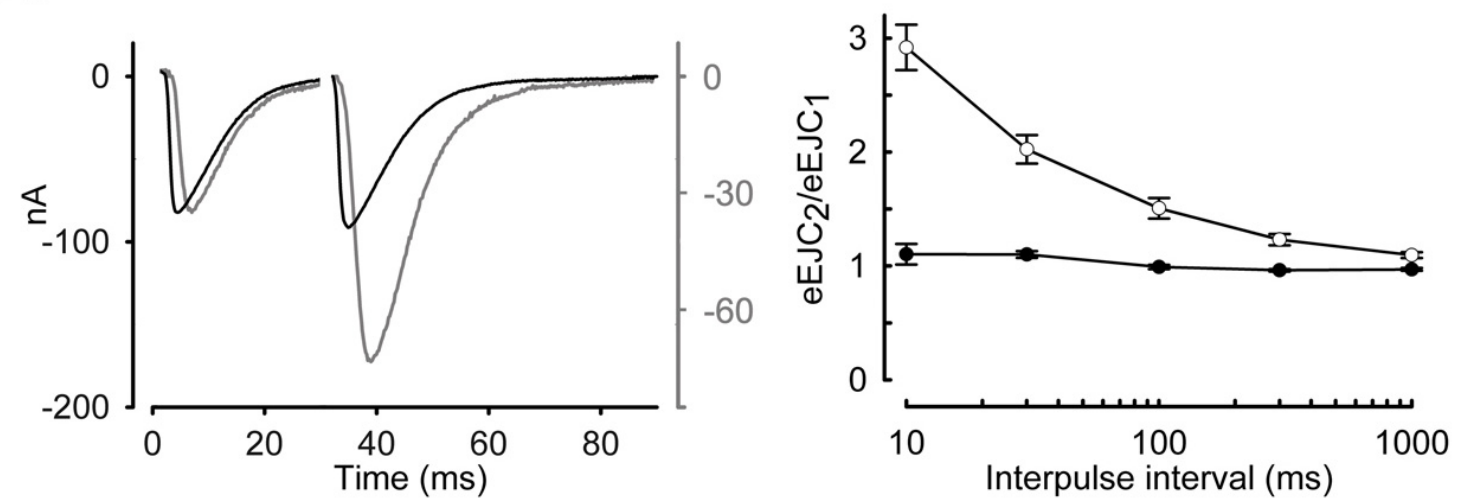

Figure 20. Changes in short-term plasticity at bruchpilot mutant NMJs.

(A) $10 \mathrm{~Hz}$ stimulation revealed transient short-term facilitation of brp mutant currents (white circles) and the absence of a frequency-dependent depression of steady-state current amplitudes compared to controls (black circles) ( $\mathrm{n} \geq 10$ per genotype at each frequency). (B) Average currents following paired-pulse stimulation at an interval of $30 \mathrm{~ms}$ normalised to the amplitude of the first pulse (control = black, brp = grey) and paired-pulse ratios at varying intervals demonstrated the pronounced potentiation at brp NMJs $[\mathrm{n}=9$ per genotype at each interval; (Kittel et al., 2006b)]. 
Previous studies have demonstrated that vesicle fusion is highly sensitive to the spacing between $\mathrm{Ca}^{2+}$ channels and vesicles at release sites (Neher, 1998). It has been calculated that doubling this distance from 25 to $50 \mathrm{~nm}$ decreases the vesicle release probability threefold (Bennett et al., 2000), and therefore the larger this distance, the more effective the slow synthetic $\mathrm{Ca}^{2+}$ buffer EGTA (Ethyleneglycol-bis( $\beta$-aminoethyl)-N,N,N',N'tetraacetic Acid) should become in suppressing release (Adler et al., 1991). A focal electrode was employed (Dudel, 1981; Pawlu et al., 2004) to track local nerve-evoked postsynaptic currents in the presence of membrane permeable EGTA-AM (tetraacetoxymethyl Ester of EGTA). And indeed, following bath application of EGTAAM the attenuation of evoked vesicle release was most pronounced at brp mutant NMJs (Fig. 21; Control: $64.2 \pm 13.8 \%$; brp: $16.7 \pm 8.8 \%$; $=6$ each; $\mathrm{p}=0.026$ ).
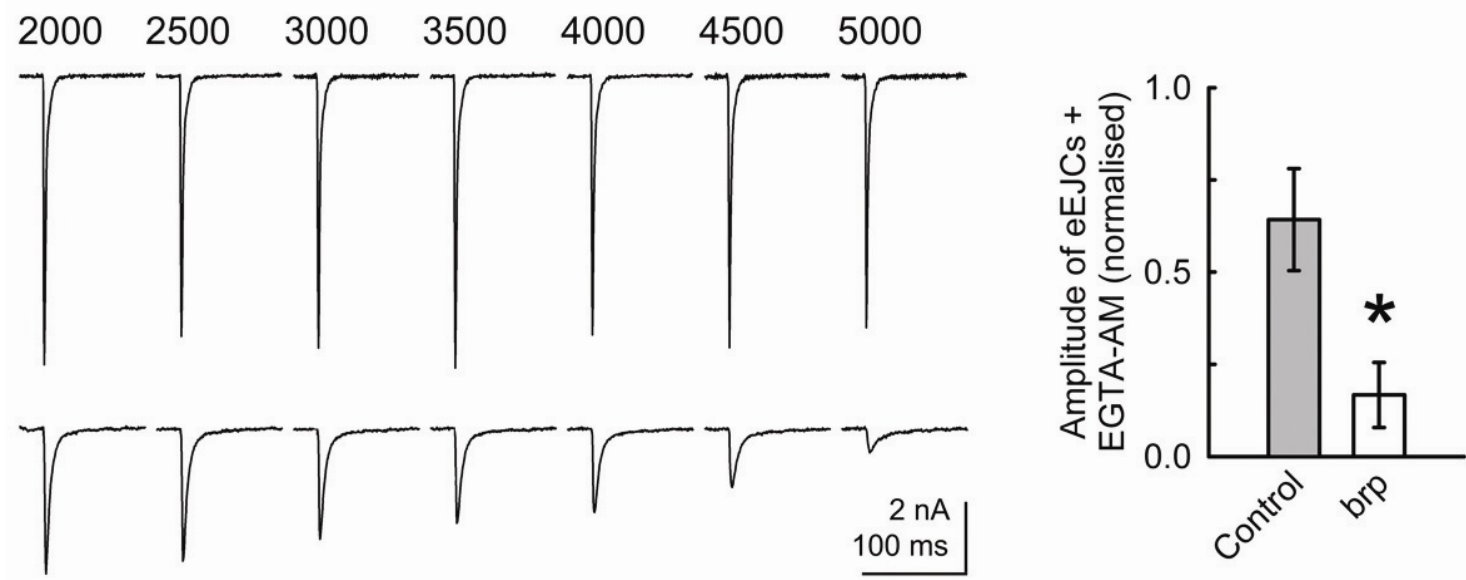

Figure 21. Increased sensitivity to the calcium buffer EGTA in bruchpilot mutants.

Examples of nerve-evoked local postsynaptic currents recorded with a focal electrode at indicated time points (in seconds) following the bath application of EGTA-AM. The bar chart illustrates the severe attenuation of current amplitudes in brp mutants $5000 \mathrm{~s}$ after EGTA-AM wash-in. The values were normalized to the initial eEJC amplitude (Kittel et al., 2006b).

Finally, the expression of $\mathrm{Ca}^{2+}$ channels was directly addressed (Kittel et al., 2006b). The presynaptically expressed N-type $\mathrm{Ca}^{2+}$ channel subunit $(\alpha 1)$ Cacophony (Cac) governs release at Drosophila neuromuscular junctions (Kawasaki et al., 2000; Kuromi et al., 2004). By utilising a functional, GFP (green fluorescent protein)-labelled variant of $\mathrm{Cac}$ [Cac ${ }^{\mathrm{GFP}}$; (Kawasaki et al., 2004)], $\mathrm{Ca}^{2+}$ channels were visualised at the larval NMJ in vivo (Rasse et al., 2005). In controls, Cac ${ }^{\text {GFP }}$ was confined to diffraction-limited 
spots, demarking $\mathrm{Ca}^{2+}$ channel clusters at presynaptic active zones [Fig. 22A; (Rasse et al., 2005)]. However, in brp mutants the $\mathrm{Ca}^{2+}$ channel expression was severely reduced both over the entire NMJ (Fig. 22B; Control: $31.1 \pm 2.4$ arbitrary units of intensity (a.u.), $\mathrm{n}=13 ;$ brp: $18.0 \pm 2.0$ a.u., $\mathrm{n}=10 ; \mathrm{p}=0.0017$ ) and at individual synapses (Fig. 22B; Control: $52.6 \pm 1.2$ a.u., $\mathrm{n}=421$ synapses; brp: $25.3 \pm 0.8$ a.u., $\mathrm{n}=320$ synapses; $\mathrm{p}<0.001$, t-test). Additionally, the $\mathrm{Cac}^{\mathrm{GFP}}$ signal appeared diffuse (Fig. 22C).
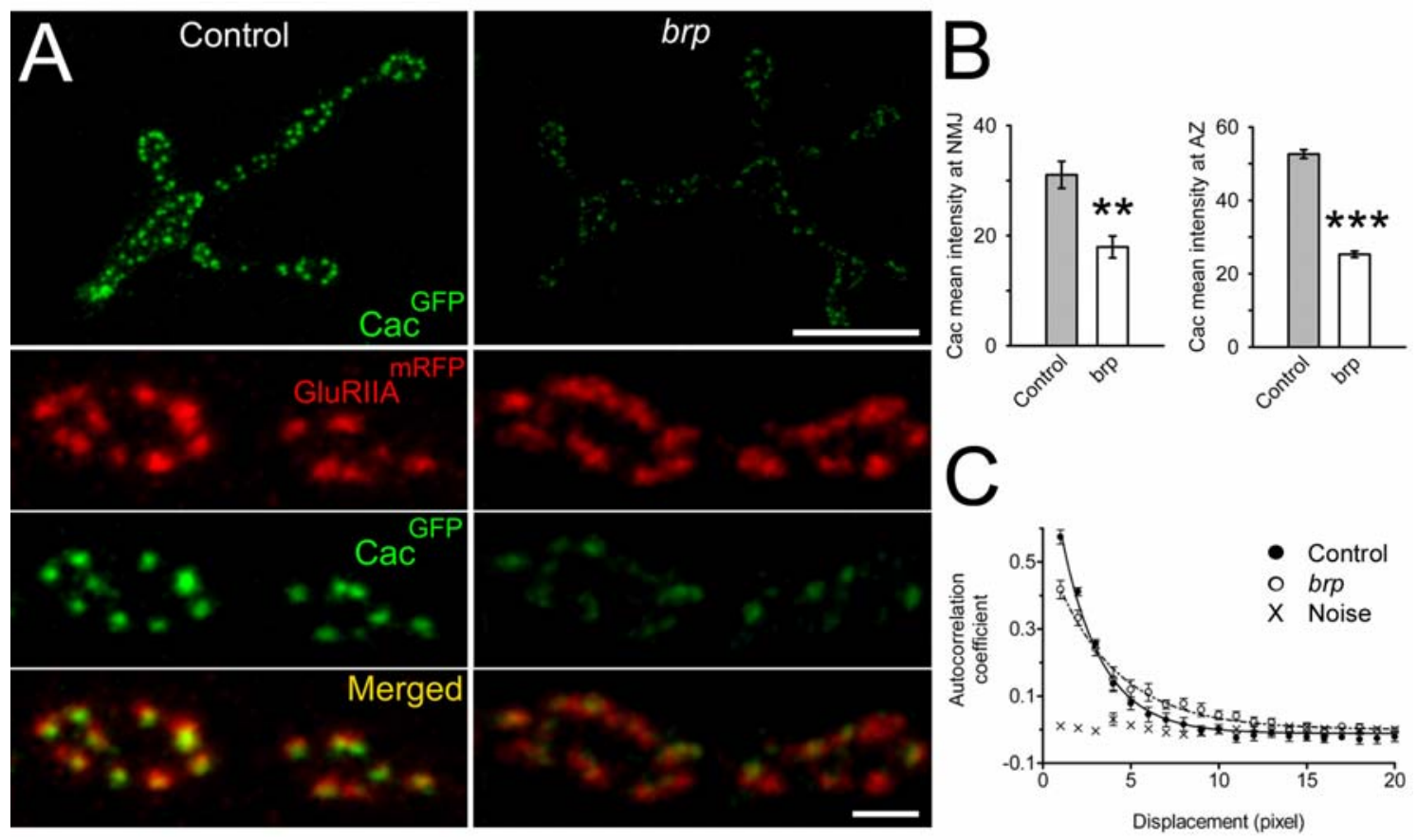

Figure 22. Mislocalisation of presynaptic calcium channels in bruchpilot mutants.

(A) Projections of confocal stacks displaying (top images; scale bar, $10 \mu \mathrm{m}$ ) and several boutons (lower images; scale bar, $2 \mu \mathrm{m}$ ) revealed the weak Cac ${ }^{\text {GFP }}$ signal at brp mutant synapses. (B) Quantification of $\mathrm{Cac}^{\mathrm{GFP}}$ intensity averaged over the entire NMJ or only synaptic areas (Kittel et al., 2006b). (C) Autocorrelation analysis of the $\mathrm{Cac}^{\mathrm{GFP}}$ signal within individual boutons demonstrated the diffuse distribution in brp mutants. Noise values were obtained from regions outside the NMJ (software courtesy of J. Sørensen, MPIBPC Göttingen).

In conclusion, brp mutants suffered from a diminished vesicle release probability due to a decrease in the density of presynaptic $\mathrm{Ca}^{2+}$ channel clusters and an increase in the average distance between $\mathrm{Ca}^{2+}$ channels and vesicles at release sites. By establishing proximity between $\mathrm{Ca}^{2+}$ channels and synaptic vesicles at the active zone Bruchpilot promotes the efficient coupling of excitation with secretion. 


\subsection{Drosophila glutamate receptor subunits GluRIIA and GluRIIB}

\subsubsection{Background and functional GFP fusions}

At the larval NMJ, the formation of synapses can be controlled by the subunit-specific expression of neuromuscular glutamate receptors [see Chapter 2.5.3.; (Petersen et al., 1997; Sigrist et al., 2000; Sigrist et al., 2002; Sigrist et al., 2003; Marrus et al., 2004)]. Thereby, the growth of individual synapses directly correlates with the entry and stable integration of glutamate receptors into the PSD from diffuse extrasynaptic pools (Rasse et al., 2005).

Two receptor complexes, composed of GluRIIC, GluRIID, and GluRIIE together with either GluRIIA, or GluRIIB, appear to co-exist within individual synapses. Structurally normal synapses are present in single mutants of gluRIIA or gluRIIB, indicating that either subunit is in principle dispensable for the formation of neuromuscular synapses (Petersen et al., 1997; DiAntonio et al., 1999; Marrus et al., 2004; Qin et al., 2005). However, in double mutants of gluRIIA\&IIB ( gluRIIA $\left.^{\text {null }} \& I I B^{\text {null }}\right)$, and similarly in gluRIIC $^{\text {null }}$, gluRIID ${ }^{\text {null }}$ and gluRIIE $E^{\text {null }}$ single mutants, embryonic lethality results from a complete absence of all glutamate receptor subunits (Petersen et al., 1997; Featherstone and Broadie, 2002; Marrus et al., 2004; Qin et al., 2005). The following chapter presents the results of an electrophysiological investigation into the differential influences of GluRIIA and GluRIIB containing receptor complexes on postsynaptic strength.

The transparency of larval body wall muscles enables the application of imaging techniques to follow the spatio-temporal sequences of glutamate receptor field formation in vivo. To receive tools with which the modes and rates of receptor turnover could be addressed throughout the development of the NMJ in alive, intact larvae, GFP fusions to receptor subunits were constructed. After several unsuccessful attempts, transgenic receptor subunits containing a mid-C-terminal GFP fusion were obtained (Rasse et al., 2005; Schmid et al., 2006). A single copy of GFP-tagged IIA (gluRIIA ${ }^{G F P}$ ) or IIB (gluRIIB $\left.{ }^{G F P}\right)$ expressed from a genomic transgene rescued gluRIIA ${ }^{\text {null }} \& I I B^{\text {null }}$ larval lethality (GluRIIA ${ }^{\text {GFP }}$ and GluRIIB ${ }^{\text {GFP }}$ rescues) and gave rise to viable adults in mendelian ratio (Rasse et al., 2005). Furthermore, the sub-cellular localisation of GFPtagged subunits was indistinguishable from untagged subunits expressed in the gluRIIA $^{\text {null }} \& I I B^{\text {null }}$ background (GluRIIA and GluRIIB rescues) and the subunit protein 
levels were similar to those at wild-type NMJs [Fig. 23; (Rasse et al., 2005)]. To assess whether the fluorescent tag influenced the synaptic physiology, TEVC recordings were made from larval muscles [Fig. 24; (Rasse et al., 2005)].

Neither GluRIIA ${ }^{\text {GFP }}$ nor GluRIIB ${ }^{\text {GFP }}$ rescues differed from their untagged counterparts in terms of mini amplitude (Fig. 24B, C; IIA:-1.26 $\pm 0.09 \mathrm{nA}, \mathrm{n}=9$; IIA $^{\text {GFP }}:-1.29 \pm$ $0.15 \mathrm{nA}, \mathrm{n}=9 ; \mathrm{p}=0.93$; IIB: $-0.7 \pm 0.04 \mathrm{nA}, \mathrm{n}=10 ; \operatorname{IIB}^{\mathrm{GFP}}: 0.66 \pm 0.03 \mathrm{nA}, \mathrm{n}=9 ; \mathrm{p}=$ 0.51 ) or frequency (Fig. 24D; IIA: $0.65 \pm 0.11 \mathrm{~Hz}, \mathrm{n}=8$; $\operatorname{IIA}^{\mathrm{GFP}}: 0.52 \pm 0.09 \mathrm{~Hz}, \mathrm{n}=9$; $\mathrm{p}=0.34$; IIB: $0.1 \pm 0.02 \mathrm{~Hz}, \mathrm{n}=10$ IIB $^{\mathrm{GFP}}: 0.05 \pm 0.01 \mathrm{~Hz}, \mathrm{n}=9 ; \mathrm{p}=0.17$ ). Similarly, the amplitude of eEJCs (Fig. 24A, C; IIA: $-113 \pm 11 \mathrm{nA}, \mathrm{n}=9$; IIA $^{\mathrm{GFP}}:-124 \pm 10 \mathrm{nA}$, $\mathrm{n}=9 ; \mathrm{p}=0.45 ;$ IIB: $-47 \pm 5 \mathrm{nA}, \mathrm{n}=10$ IIB $^{\mathrm{GFP}}:-45 \pm 4 \mathrm{nA}, \mathrm{n}=10 ; \mathrm{p}=0.97$ ) was not significantly altered by the presence of GFP in the transgenic subunit, and remained unchanged following bleaching of the GFP signal (Fig. 24E). Thus, it was concluded that the GFP tag had no detectable influence on the synaptic physiology.
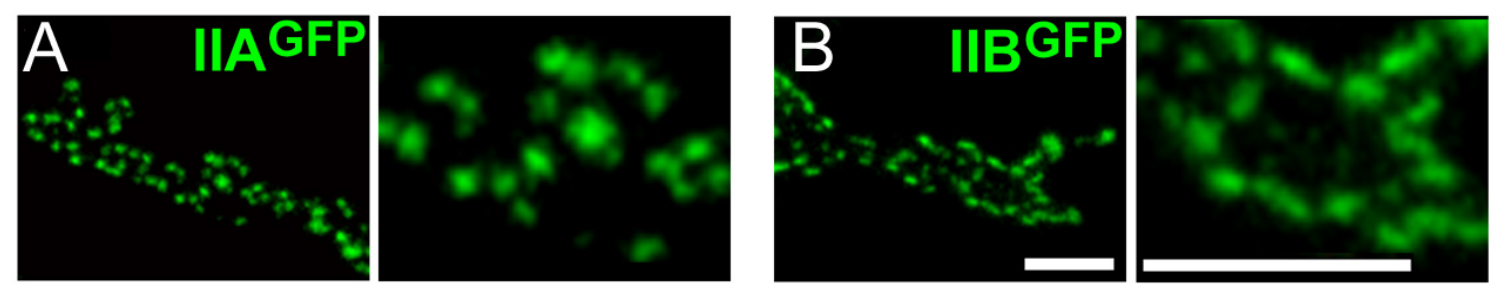

Figure 23. Expression of fluorescently tagged receptor subunits at the NMJ.

(A) GluRIIA tagged with GFP (IIA ${ }^{\text {GFP }}$ ) and (B) GFP-tagged GluRIIB (IIB ${ }^{\text {GFP }}$ ) localise to the NMJ. Scale bars, $5 \mu \mathrm{m}$. Courtesy of A. Schmid, manuscript in preparation.

\subsubsection{Differential influences on postsynaptic sensitivity}

The synaptic properties of GluRIIA ${ }^{\text {GFP }}$ rescues differed strongly from those of GluRIIB $^{\text {GFP }}$ rescues (Fig. 24). GluRIIA ${ }^{\text {GFP }}$ rescued larvae had larger individual receptor fields (IIA ${ }^{\text {GFP }}: 0.35 \pm 0.007 \mu \mathrm{m}, \mathrm{n}=550$ receptor fields, $8 \mathrm{NMJs}$; IIB ${ }^{\mathrm{GFP}}: 0.33 \pm 0.01$ $\mu \mathrm{m}, \mathrm{n}=433$ receptor fields, 9 NMJs; $\mathrm{p}=0.01$ ) and more synapses at the NMJ (IIA ${ }^{\text {GFP }}$ : $887.8 \pm 46, \mathrm{n}=20$ NMJs; IIB ${ }^{\text {GFP }}: 627.9 \pm 19, \mathrm{n}=20$ NMJs; $\mathrm{p}<0.001 ;$ A. Schmid, personal communication). Compatible with a low postsynaptic sensitivity, the amplitudes of both eEJCs $(p<0.001)$ and minis $(p<0.001)$ were smaller in GluRIIB ${ }^{\text {GFP }}$ 
rescues. Compared to GluRIIA ${ }^{\mathrm{GFP}}$ rescues, minis were only detected at very low frequencies in GluRIIB ${ }^{\mathrm{GFP}}$ rescued larvae $(\mathrm{p}<0.001)$, and this 10 -fold reduction in the rate of spontaneous release events did not correlate well with roughly $30 \%$ less individual synapses. Instead of attributing the lower frequency solely to a decrease in the number of synapses, it was therefore hypothesised that a significant fraction minis were below the detection limit at GluRIIB $^{\text {GFP }}$ rescued NMJs, leading to an overestimation of the average amplitude.

A

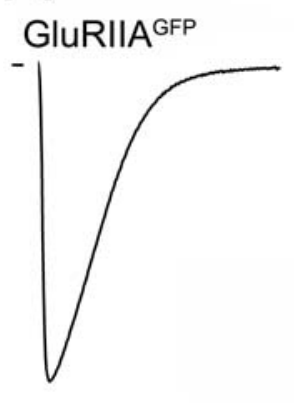

B
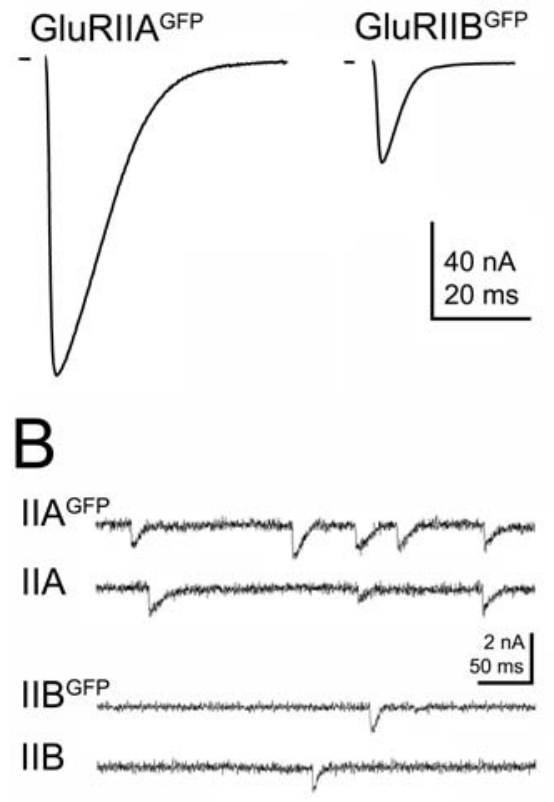

$40 \mathrm{nA}$

$20 \mathrm{~ms}$

$2 \mathrm{nA}$

$0 \mathrm{~ms}$

IIB
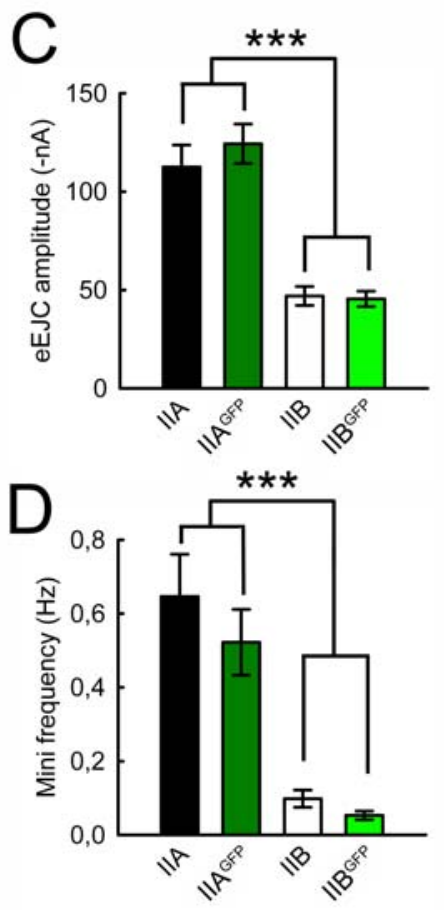

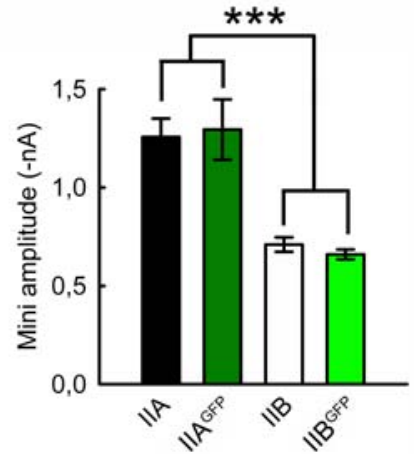

$\mathrm{E}$

Pre

Post

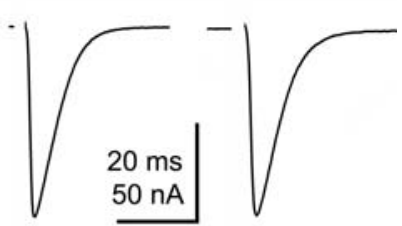

Figure 24. Effects of the glutamate receptor subunit composition on synaptic efficacy.

(A, B) Example traces of eEJCs (A) and minis (B) from larval NMJs expressing either a GFPtagged or an endogenous copy of the GluRIIA or GluRIIB subunit in the gluRIIA\&IIB double mutant background. (C, D) The GFP-tag did not influence the effect of IIA (Rasse et al., 2005) or IIB on synaptic physiology. Compared to IIB, the presence of the IIA subunit increased the amplitude of evoked and miniature EJCs (C) and elevated the mini frequency (D). (E) Bleaching of the GFP-tagged IIA subunit did not alter the amplitude of eEJCs. Shown are average traces before (pre) and after bleaching [post; 7 min after start of bleaching, 2 min after complete loss of signal; (Rasse et al., 2005)]. GluRIIB data courtesy of A. Frölich.

To obtain a superior signal-to-noise ratio and thereby address this consideration, patchclamp recordings were made from embryonic muscles (see Chapter 3.2.3.). Under these experimental conditions (Fig. 25), minis were detected at an increased average 
frequency in GluRIIB ${ }^{\mathrm{GFP}}$ rescued embryos and this rate approached GluRIIA ${ }^{\text {GFP }}$ levels (Fig. 25A; IIB ${ }^{\mathrm{GFP}}: 0.29 \pm 0.12 \mathrm{~Hz}, \mathrm{n}=7$ IIA $^{\mathrm{GFP}}$ : $0.6 \pm 0.2 \mathrm{~Hz}, \mathrm{n}=6 ; \mathrm{p}=0.234$ ). Relative to GluRIIA ${ }^{\text {GFP }}$, the average amplitude of minis at GluRIIB ${ }^{\text {GFP }}$ rescued embryonic NMJs was decreased to a larger extent than at larval NMJs recorded in TEVC (Fig. 25A; $\operatorname{IIB}^{\mathrm{GFP}}:-40 \pm 1.4 \mathrm{pA}, \mathrm{n}=7$ IIA $^{\mathrm{GFP}}:-132 \pm 13 \mathrm{pA}, \mathrm{n}=6, \mathrm{p}=0.001$ ).

A

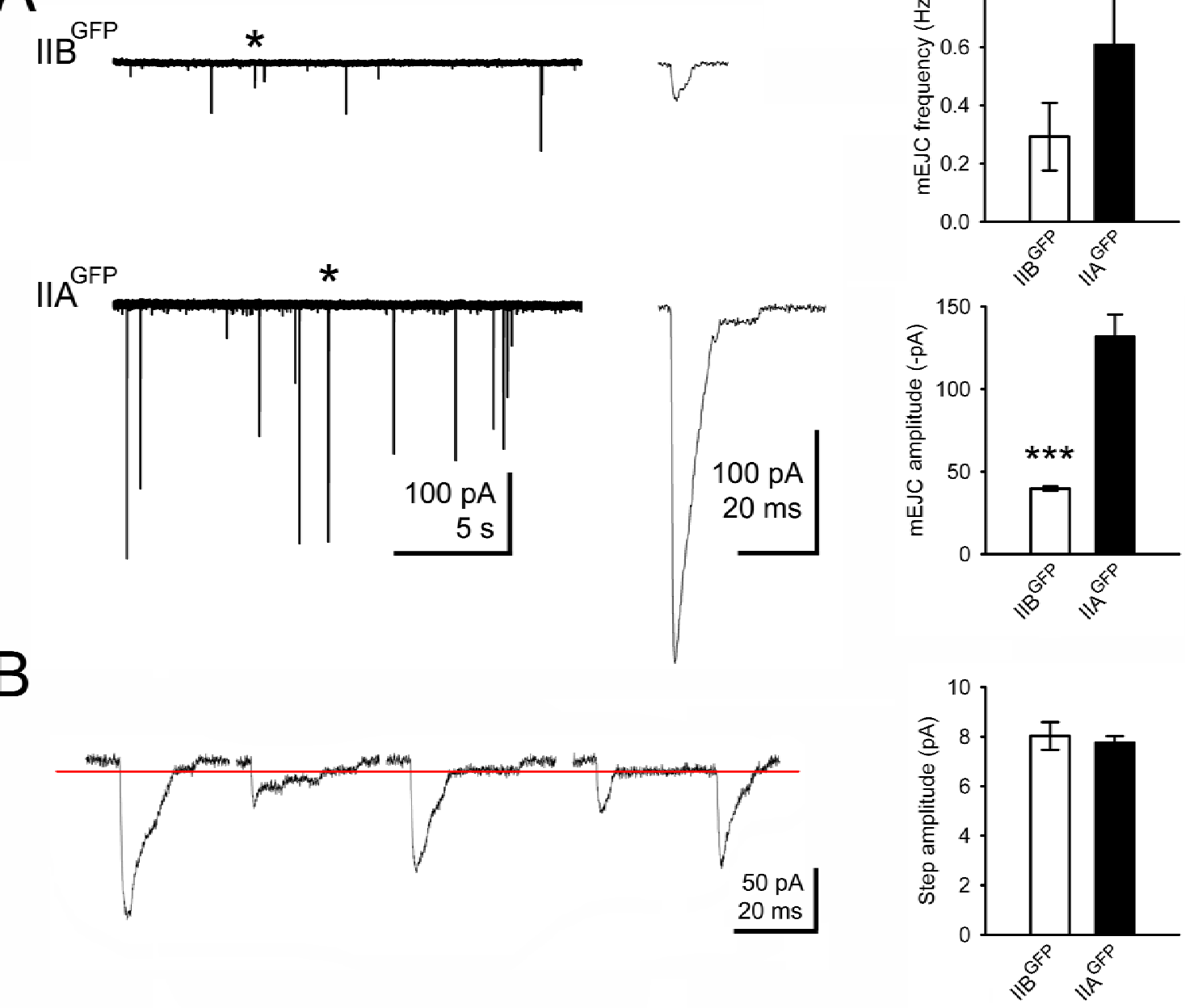

Figure 25. Receptor subunit composition determines quantal size.

(A) Example traces of minis (asterisks show expanded events) from whole-cell patch clamp recordings, and bar charts of the average mini amplitude and frequency at GluRIIA GFP and GluRIIB $^{\text {GFP }}$ rescued embryonic NMJs. (B) Examples of events displaying a stepwise decay (highlighted by red line) and quantification of the step amplitude in GluRIIA ${ }^{\text {GFP }}$ and GluRIIB ${ }^{\text {GFP }}$ rescues.

Because of the high resistance of embryonic muscles and the large unitary conductance of neuromuscular glutamate receptors, the opening (or closing) of individual receptor ion channels can be resolved in the whole-cell patch clamp configuration (Broadie and 
Bate, 1993a). Miniature EJCs often displayed a stepwise decay (Fig. 25B), and measuring the amplitude of these steps provided an opportunity to gain direct information on the channel conductance underlying the synaptic currents. The amplitude of steps was comparable between GluRIIB $^{\text {GFP }}$ and GluRIIA ${ }^{\text {GFP }}$ rescued embryos (at $-60 \mathrm{mV}$; IIB ${ }^{\mathrm{GFP}}: 8.02 \pm 0.56 \mathrm{pA}, \mathrm{n}=29{\text { steps, } 7 \mathrm{NMJs} \text {;IA }}^{\text {GFP }}: 7.76 \pm 0.26$ $\mathrm{pA}, \mathrm{n}=90$ steps, $6 \mathrm{NMJs} ; \mathrm{p}=0.53$ ) and assuming a reversal potential of $+12 \mathrm{mV}$ (Nishikawa and Kidokoro, 1995) this translates into approximately $111 \mathrm{pS}$ and $108 \mathrm{pS}$ respectively. These estimates are in good agreement with previous whole-cell recordings of junctional embryonic wild-type channels $[\sim 115 \mathrm{pA}$; (Nishikawa and Kidokoro, 1995)] and excised patch recordings of extrajunctional channels from larval muscles [wild-type, GluRIIB, and GluRIIA rescues all $\sim 120$ pS; (Heckmann and Dudel, 1995; DiAntonio et al., 1999)].

In summary, the incorporation of IIB, rather than IIA into glutamate receptor complexes led to a lower postsynaptic sensitivity at the NMJ, independent of the single channel conductance.

\subsubsection{Effects of minimal glutamate receptor expression}

Due to the prominent role of GluRIIA in both morphological and functional synaptic strengthening, and the reported presynaptic compensation for reduced postsynaptic sensitivity (see Chapter 2.5.3.), the physiological consequences of GluRIIA deprivation in the absence of GluRIIB were examined (Qin et al., 2005; Schmid et al., 2006). To achieve this situation, the gluRIIA $A^{\text {null }} \& I I B^{\text {null }}$ background was rescued by a gluRIIA genomic transgene that encoded the whole open reading frame but lacked parts of the 3'-UTR (untranslated region). Individuals of this genotype (gluRIIA ${ }^{\text {hypo }}$ ) had less than $5 \%$ of wild-type GluRIIA mRNA levels, no GluRIIB, a minimal expression of all other glutamate receptor subunits at the NMJ, and a defective assembly of the PSD (Qin et al., 2005; Schmid et al., 2006). In contrast, the molecular and structural composition of presynaptic active zones appeared unaffected (Schmid et al., 2006), and larvae were only moderately limited in mobility. In TEVC, nerve-evoked junctional currents at gluRIIA $^{\text {hypo }}$ larval NMJs were reduced to about $30 \%$ of wild-type amplitudes (Fig. 26; wt: $\left.-62 \pm 3 \mathrm{nA}, \mathrm{n}=12 ; I I A^{\text {hypo }}:-21 \pm 2 \mathrm{nA}, \mathrm{n}=11 ; \mathrm{p}<0.001\right)$, and whereas the 
amplitudes of wild-type minis ranged between the values obtained for GluRIIA and GluRIIB rescues (wt: $-1.12 \pm 0.04 \mathrm{nA}, \mathrm{n}=9 ; \mathrm{p}<0.001$ for IIB; $\mathrm{p}=0.233$ for IIA), no spontaneous events were detected at gluRIIA ${ }^{\text {hypo }}$ NMJs (Qin et al., 2005). Under these experimental settings, the event detection threshold is estimated at about $20 \%$ of the wild-type mini amplitude. It was therefore considered that more vesicles were released per action potential at gluRIIA hypo NMJs to compensate for a severe reduction of postsynaptic sensitivity. Correspondingly, an atypical paired-pulse depression was observed in gluRIIA hypo larvae, as would be expected for a synaptic connection expressing a chronic increase in presynaptic release probability [Fig. 26; paired-pulse ratio at $19.5 \mathrm{~ms}$ interval; wt: $1.18 \pm 0.04, \mathrm{n}=7 ; I I A^{\text {hypo }}: 0.83 \pm 0.05, \mathrm{n}=11 ; \mathrm{p}=0.003$; (Schmid et al., 2006)].

\section{A}

wt IIA hypo
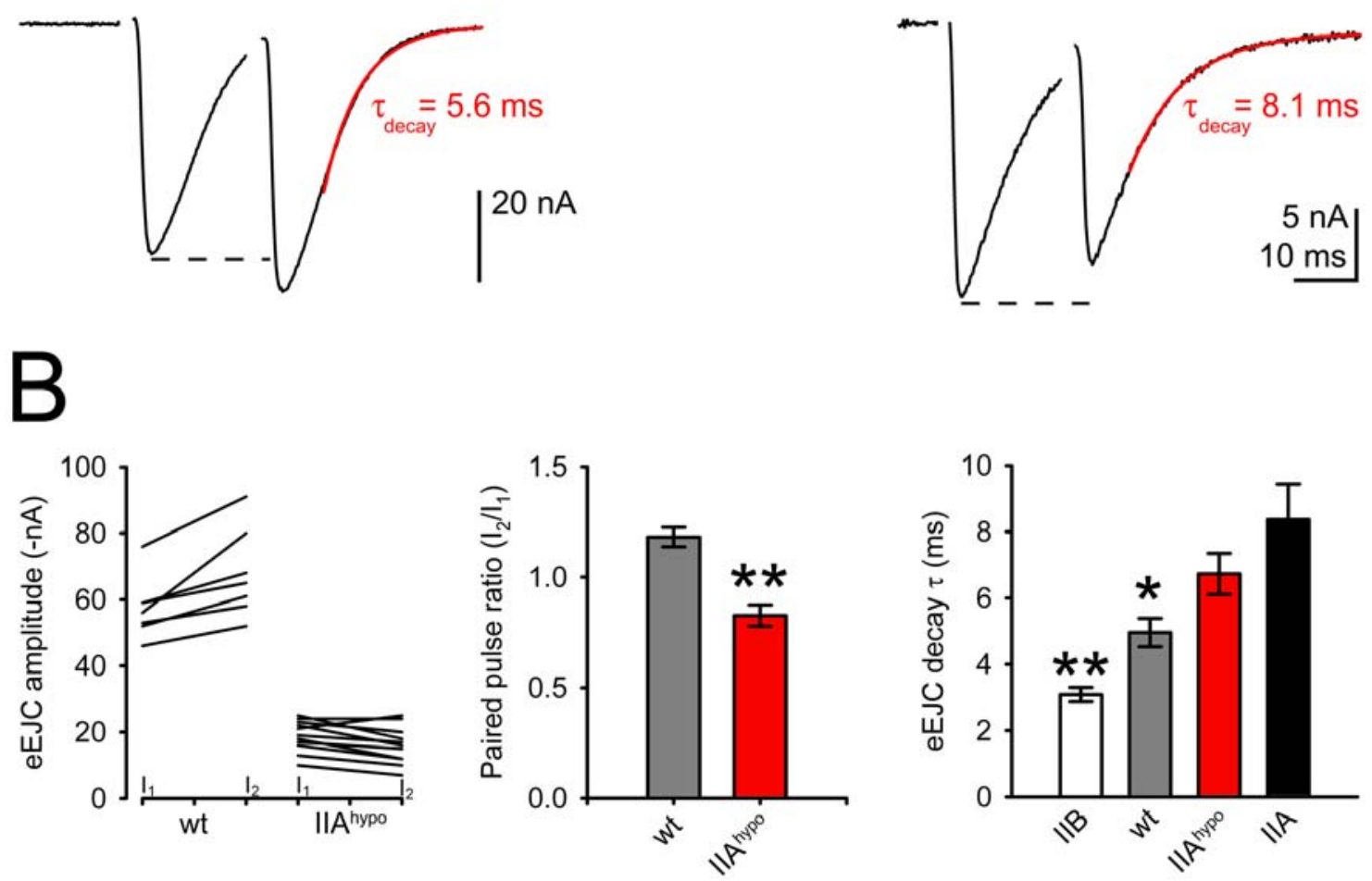

Figure 26. Minimal glutamate receptor expression leads to presynaptic compensation.

(A) Average traces of eEJCs recorded from larvae in TEVC. gluRIIA ${ }^{\text {hypo }}$ NMJs show atypical paired-pulse depression and prolonged decay $\tau$ (Schmid et al., 2006). (B) Quantification of the paired-pulse ratio and decay $\tau$. The subunit composition of receptors correlates with the decay time constant of eEJCs. 
In addition, the decay time constant $(\tau)$ of evoked responses was increased at gluRIIA ${ }^{\text {hypo }}$ NMJs [Fig. 26; $\tau$ wt: $4.96 \pm 0.42 \mathrm{~ms}, \mathrm{n}=12 ; \tau$ delta: $6.73 \pm 0.62 \mathrm{~ms}, \mathrm{n}=11$; $\mathrm{p}=0.013$; (Schmid et al., 2006)]. This may reflect atypical functional properties of the remaining glutamate receptors, or the absence of receptors containing GluRIIB, which desensitise more rapidly than receptors composed of GluRIIA (DiAntonio et al., 1999). Accordingly, eEJCs of GluRIIA rescued larvae decayed with a similar average time constant as in the gluRIIA ${ }^{\text {hypo }}$ situation ( $\tau$ IIA: $8.37 \pm 1.07 \mathrm{~ms}, \mathrm{n}=9 ; \mathrm{p}=0.494$ ), whereas eEJCs at GluRIIB rescued NMJs decayed more rapidly (Fig. 26; $\tau$ IIB: $3.09 \pm$ $0.21 \mathrm{~ms}, \mathrm{n}=10 ; \mathrm{p}=0.002$ to $I I A^{\text {hypo }}$ ). 


\subsection{Drosophila Basigin}

\subsubsection{Maintenance of synaptic domains by perisynaptic Basigin}

Drosophila Basigin (Bsg) is the homologue of the mammalian transmembrane protein CD147/EMMPRIN/Basigin (Curtin et al., 2005). Bsg is a small type I transmembrane protein of the IgG superfamily. It is composed of two extracellular Ig-like domains, a transmembrane domain, and a short cytoplasmic tail. Bsg is expressed at the larval NMJ, where it co-localised with Dlg postsynaptically in the SSR (Fig. 27A, B). Similar to perisynaptic transmembrane proteins involved in the structural control of synaptic terminals, such as Dlg or Fas II, Basigin also localised to periactive zones [Fig. 27C; (Budnik, 1996; Sone et al., 2000; Besse et al., 2006)].
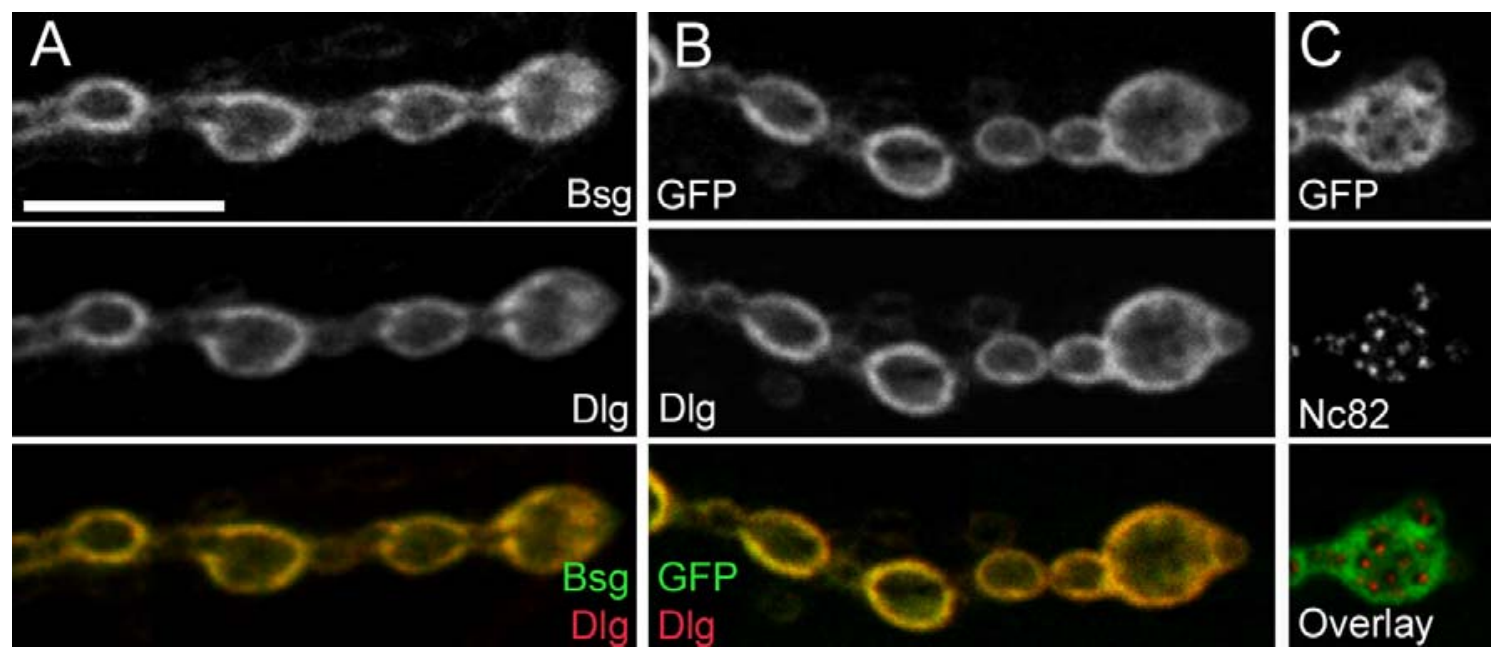

Figure 27. Basigin accumulation at the NMJ.

(A, B) NMJs of a wild-type (A) and a larva heterozygous for GFP-tagged Bsg (B) double stained with antibodies against either Bsg (green in A) or GFP (green in B) and Dlg (red in A and B) showed strong co-localisation of Bsg and Dlg. (C) Presynaptic expression of GFPtagged Bsg using a panneuronal driver line (elav-Gal4/+; UAS-GFP-bsg/+), double stained with anti-GFP (green) and the active zone label Nc82 (red), demonstrated the exclusion of Bsg from active zones. Scale bar, $10 \mu \mathrm{m}$ (Besse et al., 2006).

To test the function of Basigin, mutant alleles of the gene encoding Bsg were generated by transposon-mediated mutagenesis (Besse et al., 2006). In basigin mutant larvae the Bsg signal at the NMJ was strongly reduced, and although the axonal targeting to the muscle appeared normal, individual synaptic boutons were significantly enlarged 
(Fig.28, 29; Control type 1b: $5.08 \pm 5.5 \mu \mathrm{m}$ s.d., $\mathrm{n}=114$; $b s g$ type $1 \mathrm{~b}: 9.6 \pm 6.2 \mu \mathrm{m}$ s.d., $\mathrm{n}=143 ; \mathrm{p}<0.001$, t-test). This phenotype was accompanied by an approximately $40 \%$ decrease in the number of type $1 \mathrm{~b}$ boutons at the NMJ 6/7 (p<0.001, t-test), without a change in the muscle size. As a near complete rescue of this dual effect could only be achieved by re-expressing wild-type Bsg both pre- and postsynaptically (elav- and mhcGal4 drivers), it would appear that Basigin function is required in both pre- and postsynaptic compartments, to control the formation and growth of synaptic boutons during the expansion of the NMJ (Besse et al., 2006). Similar to the Ig-CAM Fas II (Schuster et al., 1996a), Basigin promotes cell-cell adhesion (Besse et al., 2006), and may thus exert its influence on the structure of the NMJ through trans-synaptic homophilic interactions between the extracellular domains of pre- and postsynaptic molecules.

Whereas both the molecular composition and ultrastructure of presynaptic active zones and postsynaptic densities appeared intact at bsg mutant NMJs, the distribution of synaptic vesicles within axon terminals was heavily altered [Fig. 28; (Besse et al., 2006)].
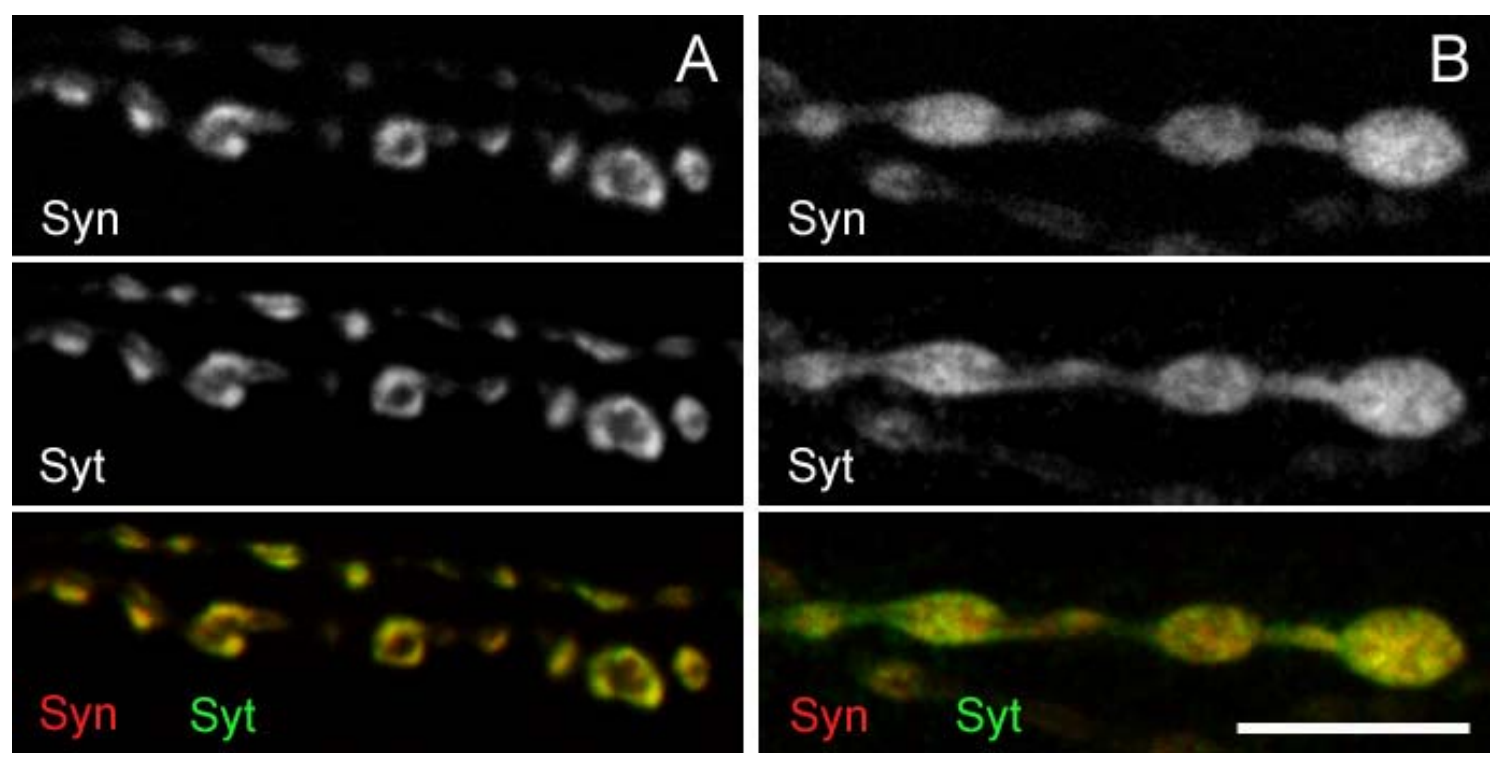

Figure 28. Diffuse distribution of synaptic vesicles in basigin mutant boutons.

(A, B) NMJs of wild-type (A) and bsg mutant (B) larvae expressing a GFP-tagged synaptotagmin (Syt) construct, double stained with anti-Synapsin (Syn; red) and anti-GFP (green). In contrast to the cortical staining pattern of control boutons, bsg mutant terminals displayed evenly distributed vesicle labels. Scale bar, $10 \mu \mathrm{m}$ (Besse et al., 2006). 
The synaptic vesicle protein synaptotagmin and the vesicle-associated protein synapsin can be used as markers of synaptic vesicles in Drosophila (Klagges et al., 1996; Zhang et al., 2002). In wild-type larvae, vesicles labelled by these proteins were strongly enriched at the cortex of synaptic boutons, and largely excluded from the central core [Fig. 28A; see also (Estes et al., 1996)]. In contrast, the preferential association of vesicles with the bouton cortex was lost in bsg mutant larvae, and synaptic vesicles were scattered throughout the presynaptic terminals (Fig. 28B). Basigin therefore appears to play a specific role in regulating the spatial distribution of synaptic vesicles, and restricting their localisation to cortical regions of synaptic boutons.

To assess the recycling capacity of the ectopic vesicles in bsg boutons, FM1-43 labelling assays were performed (Besse et al., 2006). FM1-43 is a styryl dye which is incorporated into synaptic vesicles upon endocytosis. At wild-type boutons, the stimulation of exo- and endocytosis by application of high extracellular $\mathrm{K}^{+}$ concentrations in the presence of the dye, resulted in a peripheral ring of incorporated FM1-43 (Fig. 29A). It was previously suggested that the synaptic vesicles labelled by this process represent the recycling pool of vesicles, localised to cortical regions underlying the plasma membrane (Kuromi and Kidokoro, 1998). In contrast, stimulation of basigin mutants resulted in the dye filling more central regions of synaptic boutons, indicating that a proportion of newly endocytosed membranes were inappropriately targeted to the interior of the bouton (Fig. 29B, C). Nonetheless, both the cortical label of controls, and the entire bouton of bsg mutants could be de-stained, following further $\mathrm{K}^{+}$stimulation in the absence of FM1-43. This illustrates that both cortically localised, and aberrantly distributed vesicles could be mobilised for release. 

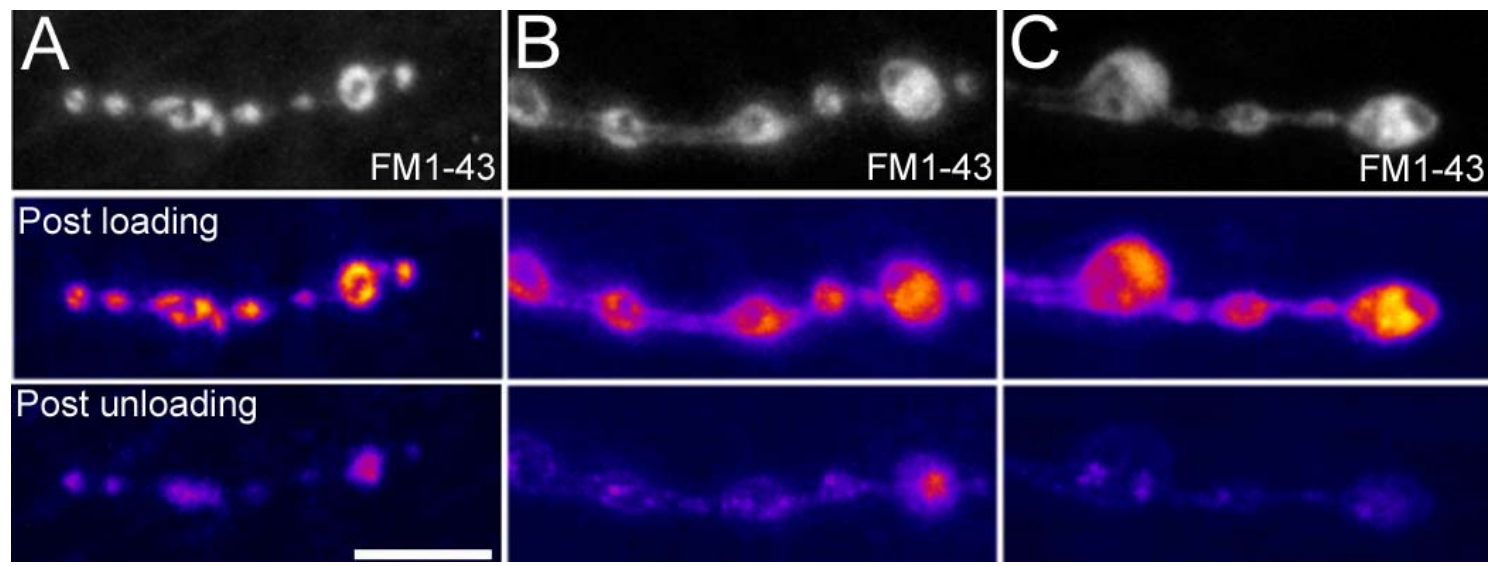

Figure 29. Altered recycling pathway of vesicles at basigin mutant synapses.

(A-C) Grey scale and colour images following activity-dependent loading (upper panels and centre panels) and unloading (lower panels) of wild-type (A) and bsg mutant boutons (B, C). Both the cortical label of controls (upper and centre panels in A), and the central label of bsg mutant boutons (upper and centre panels in B and C) were lost following de-staining (lower panels).Scale bar, $10 \mu \mathrm{m}$ (Besse et al., 2006).

\subsubsection{Physiology of basigin mutant junctions}

To address whether these changes in the distribution and recycling of synaptic vesicles were linked to functional changes in neurotransmitter release, we recorded postsynaptic currents at the larval NMJ in the two-electrode voltage clamp configuration (Besse et al., 2006). As depicted in Figure 30, the frequency of spontaneous vesicle release was significantly elevated in basigin mutants (Control: $1.90 \pm 0.23 \mathrm{~Hz}, \mathrm{n}=10$; bsg: $3.29 \pm$ $0.39 \mathrm{~Hz}, \mathrm{n}=10 ; \mathrm{p}=0.002)$, and such minis often occurred clustered in 'exocytotic bursts'. Furthermore, the amplitude of minis was increased above wild-type levels (Control: $-0.8 \pm 0.03 \mathrm{nA}, \mathrm{n}=10$; bsg: $-1.02 \pm 0.05 \mathrm{nA}, \mathrm{n}=10 ; \mathrm{p}=0.008$ ). 

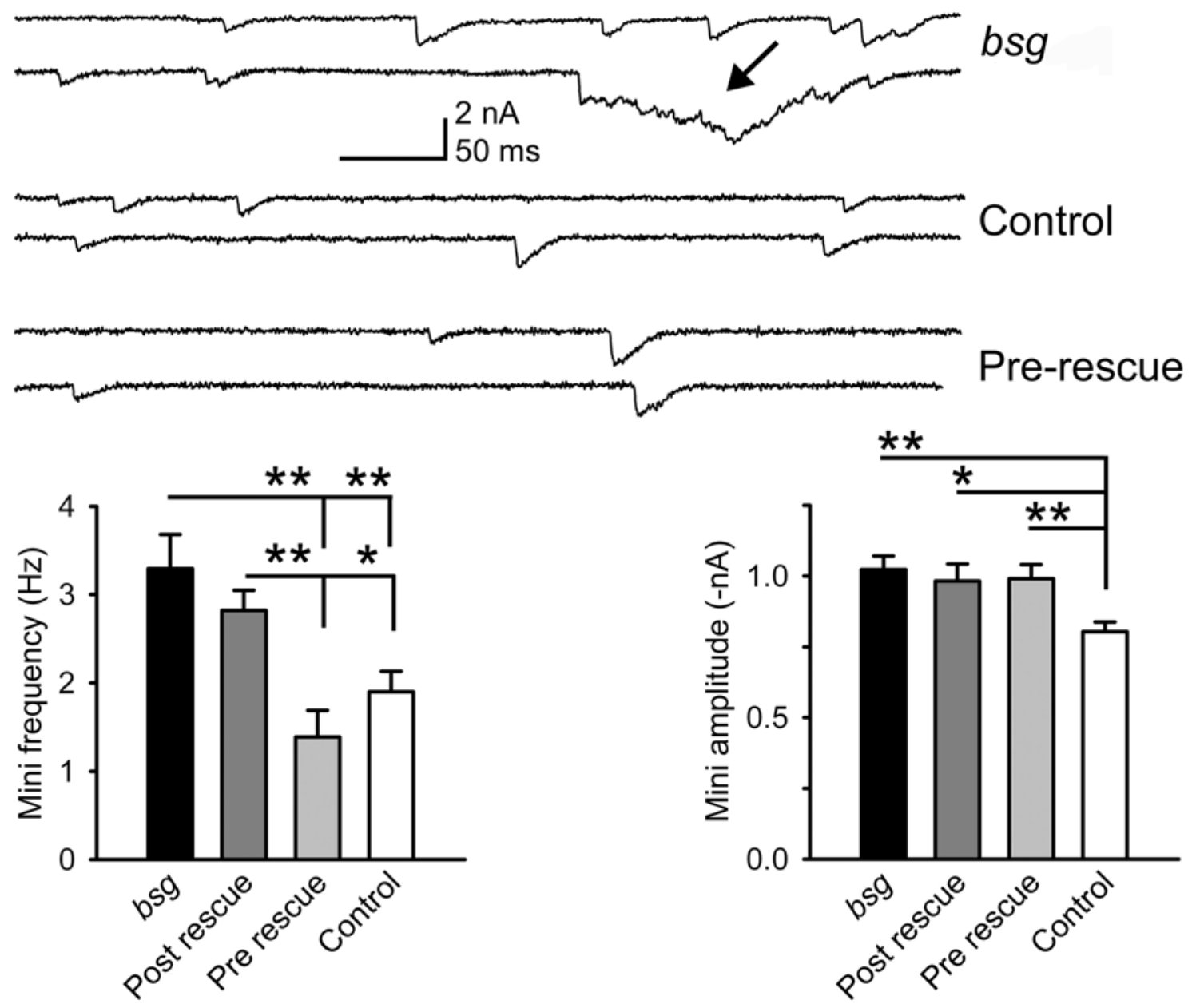

Figure 30. Presynaptic loss of Basigin elevates the frequency of spontaneous release.

Representative traces and quantification of mini frequency and amplitude. The frequency of spontaneous release was elevated at bsg mutant NMJs, often occured in 'exocytotic bursts' (arrow), and could be rescued by pre- and not postsynaptic bsg re-expression. The average mini amplitude was increased in bsg mutants, and solely pre- or postsynaptic re-expression failed to restore control levels (Besse et al., 2006).

The average amplitude of nerve-evoked EJCs was also increased at bsg mutant NMJs (Fig. 31A; Control: $-98.7 \pm 7.3 \mathrm{nA}, \mathrm{n}=10$; bsg: $-133.9 \pm 11.2 \mathrm{nA}, \mathrm{n}=10 ; \mathrm{p}=0.026$ ), and may in part be attributed to an elevated postsynaptic sensitivity, as illustrated by the increased amplitude of minis. However, the temporal profile of mutant eEJCs was strikingly lengthened, and reflected a dramatic increase in the contribution of an asynchronous release component (Fig. 31A). Indeed, a near eight-fold elevation of the charge transferred to the postsynapse following exocytosis, was observed in bsg mutants upon initial nerve stimulation. Notably, this value decreased progressively following further low frequency stimulation, which may reflect the exhaustion of the abnormally recruited vesicles responsible for this atypical asynchronous release 
component (Fig. 31B; Average charge of 15 consecutive sweeps in control: $0.99 \pm 0.02$ $\mathrm{nC}, \mathrm{n}=10 ;$ bsg: $4.72 \pm 0.26 \mathrm{nC}, \mathrm{n}=10 ; \mathrm{p}<0.001)$.

A

bsg

Control Pre-rescue
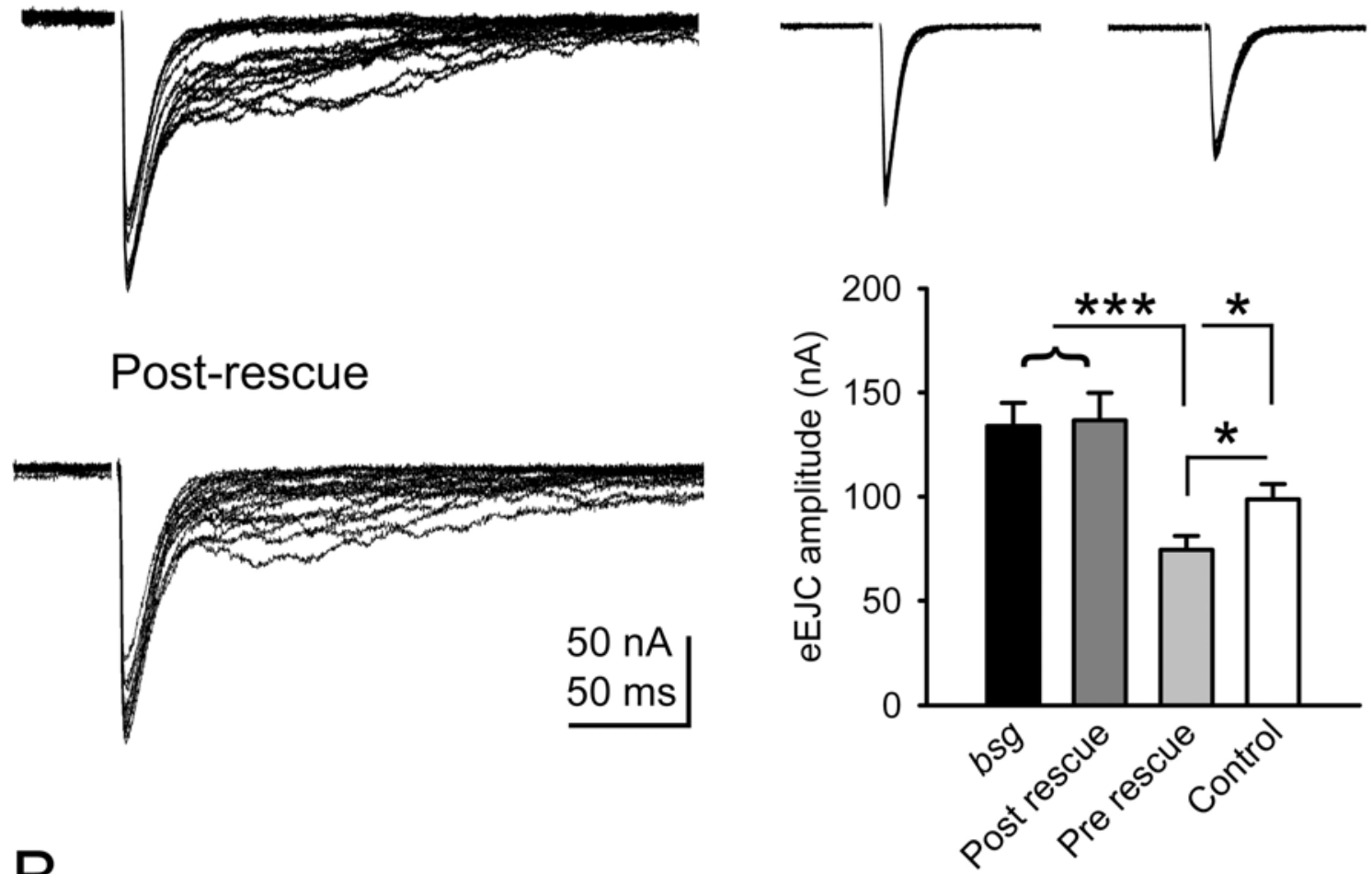

B

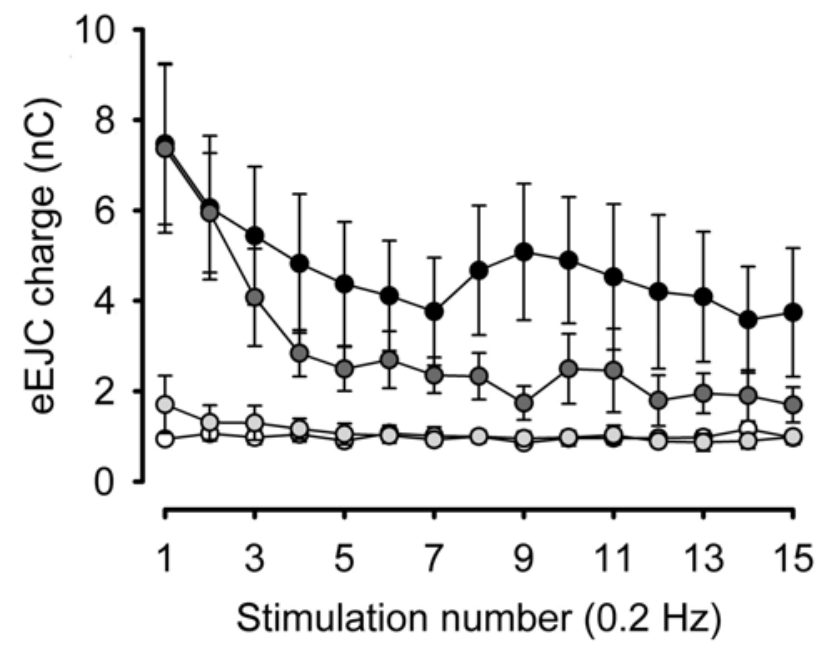

$\longrightarrow b s g$

- - Post-rescue

-0 Control

$\multimap-$ Pre-rescue

Figure 31. Excessive asynchronous evoked transmitter release in basigin mutants.

(A) Example traces of eEJCs following 15 stimuli at $0.2 \mathrm{~Hz}$, and average amplitudes. (B) Presynaptic loss of Bsg elevated asynchronous vesicle release, reflected by the larger charge carried by eEJCs of both bsg mutants and postsynaptic rescues (Besse et al., 2006). 
To test whether the down-regulation of Bsg within presynaptic terminals was responsible for the observed release defects, a wild-type copy of bsg was expressed in the presynaptic compartment of mutant larvae (elav-GAL4 driver). This approach abolished both the excessive asynchronous evoked release (Fig. 31A, B; Average charge: $1.07 \pm 0.06 \mathrm{nC}, \mathrm{n}=11 ; \mathrm{p}=0.481$ to control), and the high frequency of spontaneous release (Fig. 30; $1.39 \pm 0.30 \mathrm{~Hz}, \mathrm{n}=10 ; \mathrm{p}=0.076$ to control). Interestingly, the presynaptic re-expression of bsg even decreased the amplitude of eEJCs below wild-type levels, indicating a dose-dependent role of presynaptic Bsg in restricting release (Fig. 31A; $-74.4 \pm 6.7 \mathrm{nA}, \mathrm{n}=11 ; \mathrm{p}=0.018$ ). In contrast, postsynaptic re-expression of $b s g$ ( $m h c-G A L 4$ driver) neither rescued the asynchronous component of evoked release (Fig 31A, B; Average charge: $2.94 \pm 0.42 \mathrm{nC}, \mathrm{n}=9$; $\mathrm{p}<$ 0.001 to control), nor restored the moderate mini frequency (Fig. 30; $2.82 \pm 0.23 \mathrm{~Hz}, \mathrm{n}$ $=8 ; \mathrm{p}=0.033$ to control). As both pre- and postsynaptic re-expression failed to restore the wild-type amplitude of minis (Fig. 30; Presynaptic rescue: $-0.99 \pm 0.05 \mathrm{nA}, \mathrm{n}=10$, $\mathrm{p}=0.005$ to control; Postsynaptic rescue: $-0.98 \pm 0.06 \mathrm{nA}, \mathrm{n}=8, \mathrm{p}=0.029$ to control), Basigin may be required in both compartments for normal mini amplitudes.

In conclusion, loss of presynaptic Basigin increases the frequency of spontaneous release, and provokes atypical and dramatic asynchronous evoked release.

\subsubsection{Association of Basigin with the actin network}

Several studies have suggested that modulations of the presynaptic actin cytoskeleton may regulate the spatial organisation and mobilisation of synaptic vesicle pools (Wang et al., 1996; Dillon and Goda, 2005). Furthermore, the depolymerisation of actin filaments has been shown to specifically impair vesicle trafficking to and from the reserve pool at the Drosophila NMJ (Kuromi and Kidokoro, 1998; Delgado et al., 2000; Steinert et al., 2006). Given that Drosophila Basigin has been implicated in the regulation of cellular architecture, possibly by modulating the cytoskeleton (Curtin et al., 2005), the integrity of the actin-based cytoskeleton was investigated at bsg mutant NMJs (Besse et al., 2006).

It was previously reported, that $\alpha$-Spectrin accumulates at the larval NMJ and is closely associated with the actin-rich cytoskeleton (Featherstone et al., 2001). Although $\alpha-$ 
Spectrin was mainly enriched in the postsynaptic compartment, surrounding the bouton and extending beyond the SSR, it was also distributed cortically within the bouton (Fig. 32, A-B1). In wild-type larvae, a ring-like label of $\alpha$-Spectrin marked the edges, and not the centres of synaptic boutons (Fig. 32A, A1). In contrast, bsg mutants also had aggregates of $\alpha$-Spectrin in the bouton lumen (Fig. 32B, B1).
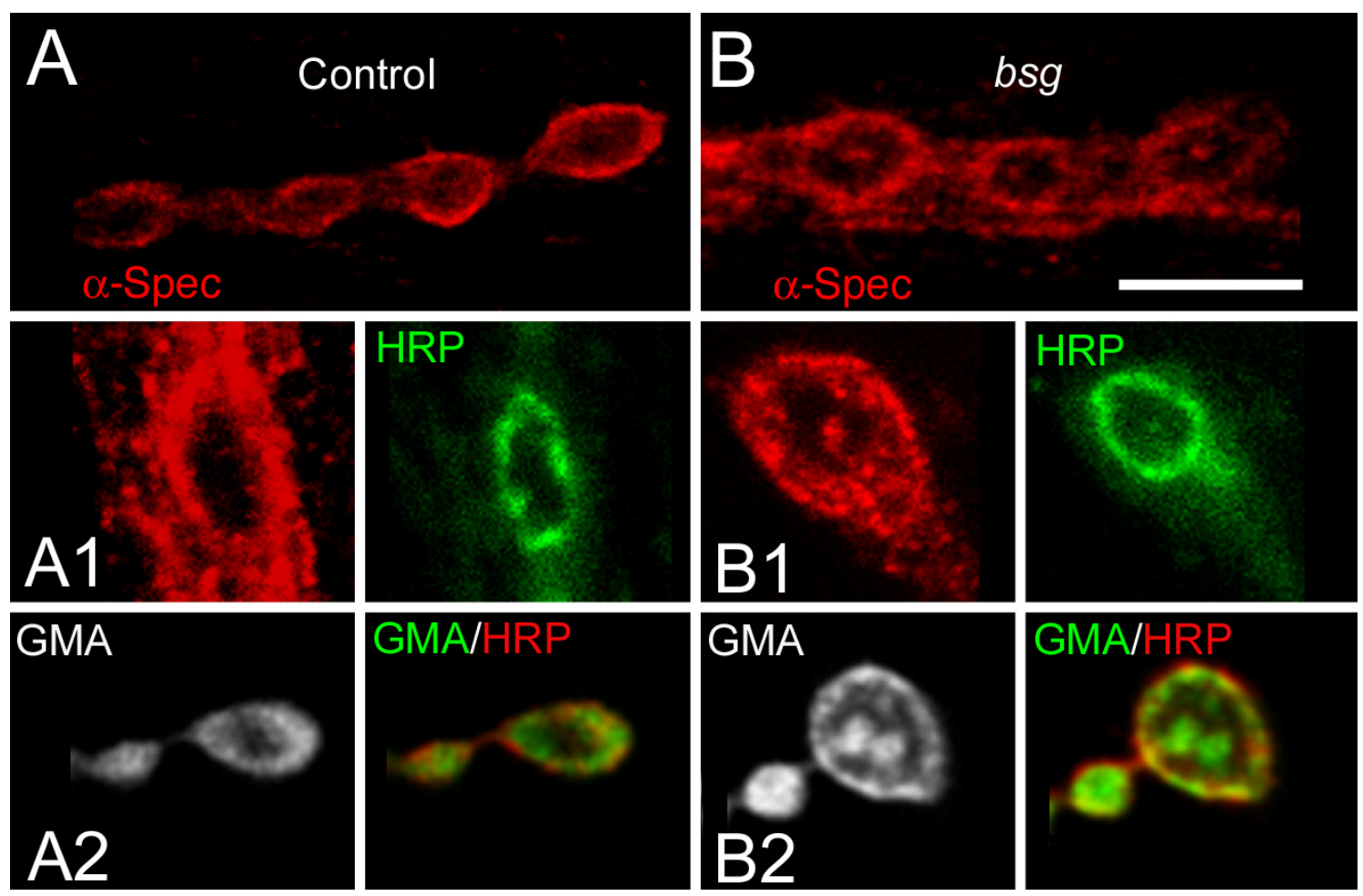

Figure 32. The distribution of cytoskeletal markers is disrupted in basigin mutants.

(A, B) Wild-type (A) and bsg mutant NMJs (B) stained with anti- $\alpha$-Spectrin (red). (A1, B1) Costaining with anti-HRP (green) illustrated the atypical HRP and Spectrin positive aggregates in the bouton centre of basigin mutants (B1). (A2, B2) Control (A2) and mutant (B2) boutons expressing an UAS-inducible fusion of GFP with the F-actin binding domain of Moesin (green), under the control of the elav-Gal4 driver, stained with anti-HRP (red). Scale bars, $10 \mu \mathrm{m}$ in (A, B) and $5 \mu \mathrm{m}$ in [A1-B2; (Besse et al., 2006)].

In order to visualise the actin cytoskeleton more directly and exclusively label the presynaptic network, the F-actin-binding domain of Moesin was fused to GFP (GFPGMA) and specifically expressed in neurons using the elav-GAL4 driver (Dutta et al., 2002). GFP-GMA specifically accumulated at the cortex of wild-type synaptic boutons and was surrounded by HRP-positive membranes (Fig. 32 A2). Although the cortical 
actin network could still be detected at the periphery of bsg mutant boutons, clusters of F-actin filaments were also present in the central regions of boutons (Fig. 32 B2).

Taken together, these data indicate that the presynaptic actin-based cytoskeleton is inappropriately organised in basigin mutants. Interestingly, the internal $\alpha$-Spectrin aggregates in mutant boutons frequently co-localised with HRP epitopes, normally restricted to the presynaptic membrane (Fig. 32 B1). Thus, the observed alterations of the actin cytoskeleton in basigin mutants may be associated with sorting defects between plasma membrane and inner endocytotic compartments. 


\section{$5 \underline{\text { Discussion }}$}

\subsection{Bruchpilot}

\subsubsection{Bruchpilot and $\mathrm{Ca}^{2+}$ channels}

The results reported in this thesis demonstrate that the Bruchpilot protein is required for both the structural and functional integrity of active zones in Drosophila. By promoting the clustering of $\mathrm{Ca}^{2+}$ channels at vesicle release sites, BRP would ensure the efficient coupling of $\mathrm{Ca}^{2+}$-dependent excitation with neurotransmitter secretion. It is conceivable that BRP fulfils a structural role by contributing to the assembly of the cytomatrix at the active zone, and either directly or indirectly interacts with active zone components such as $\mathrm{Ca}^{2+}$ channels, to regulate their spatial arrangement in the presynaptic membrane, possibly by mediating their integration into a restricted number of active zone slots (Cao et al., 2004). Related mechanisms may underlie functional impairments of mammalian central synapses lacking active zone components (Altrock et al., 2003) and natural physiological differences between synapse types (Rozov et al., 2001).

In several synaptic systems, both a fast and a slow component of exocytosis have been described (Sakaba and Neher, 2001; Hallermann et al., 2003; Schneggenburger and Neher, 2005). One interpretation of this heterogeneous response of vesicles to $\mathrm{Ca}^{2+}$ influx is that differences in the distance between $\mathrm{Ca}^{2+}$ channels and vesicles lead to heterogeneity in fusion kinetics [Fig. 33B; (Neher, 1998)]. The fast component recovers slowly and is believed to owe its properties to vesicles attached to a matrix tightly associated with $\mathrm{Ca}^{2+}$ channels (Sakaba et al., 2005), whereas the slow component recovers rapidly (Sakaba and Neher, 2001) and is thought to be important for sustaining vesicle release during tetanic stimulation. In agreement with this concept, the absence or impairment of such a matrix at brp mutant synapses has a profound effect on vesicle release at low stimulation frequencies, but this effect subsides as the frequency increases (Fig. 20A). The sustained frequency of minis could be explained if spontaneous fusion events arise from the slow release component (Trommershäuser et al., 2003) or a pathway independent of evoked vesicle fusions (Sara et al., 2005). 
A
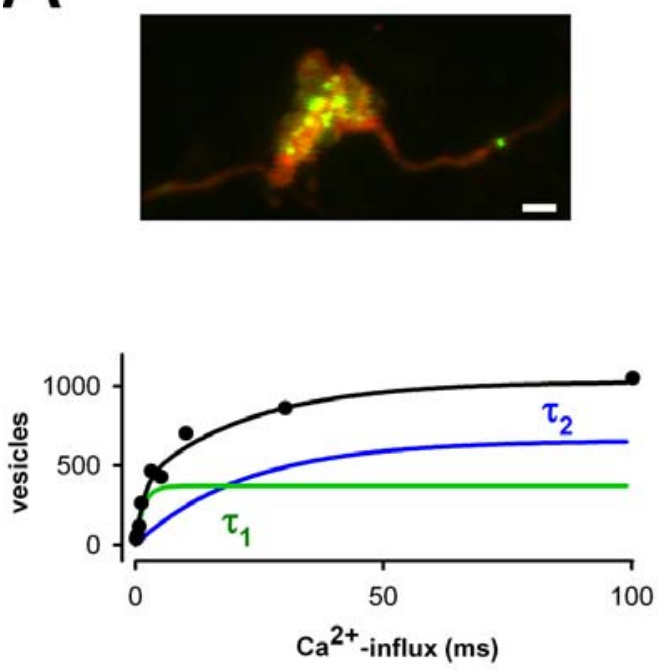

B

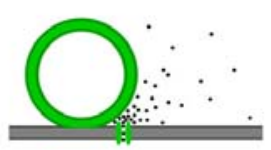

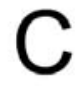

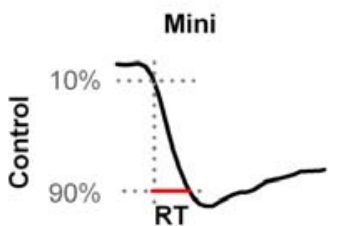

cEPSC
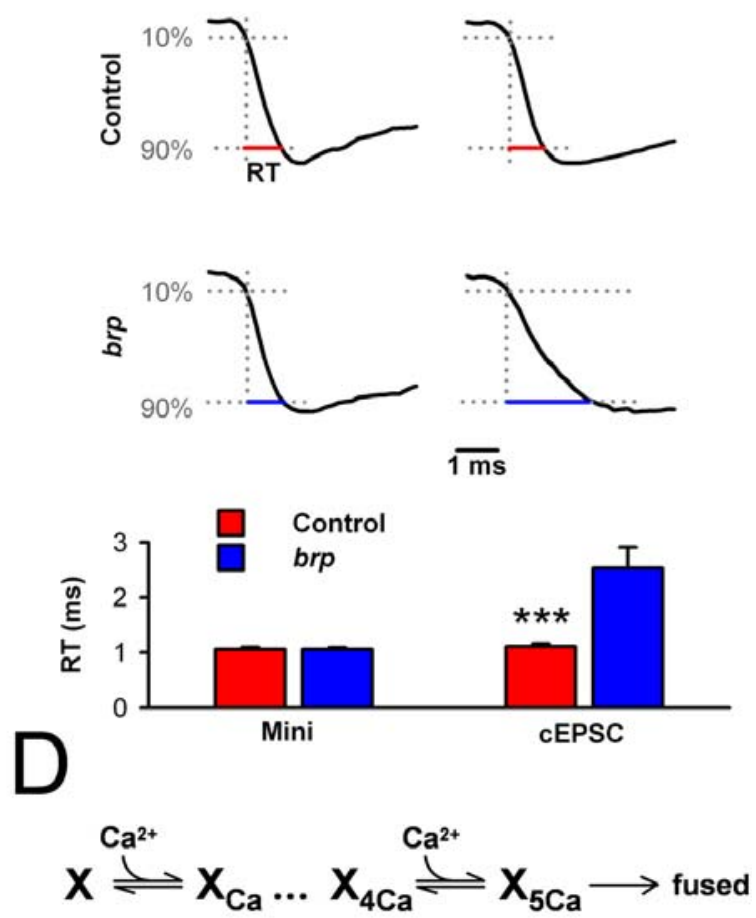

Figure 33. Vesicle pools and release kinetics.

(A) Confocal image of a mossy fibre bouton from a hippocampal granule cell in a four-weekold organotypic slice culture from a neonatal mouse two days after transfection via single-cell electroporation with CAST1-EGFP (green) and DsRed (red) cDNA. Since CAST accumulates within active zones (Hagiwara et al., 2005) the green spots probably mark individual active zones. Functionally, two pools of vesicles have been identified in mossy fibre boutons (lower panel). A fast pool of vesicles (green) fuses with a time-constant of $\tau_{1} \approx 2 \mathrm{~ms}$ and a slow pool fuses with a time-constant of $\tau_{2} \approx 20 \mathrm{~ms}$ upon $\mathrm{Ca}^{2+}$ influx (Hallermann et al., 2003). Scale bar $2 \mu \mathrm{m}$. (B) One interpretation of the heterogeneity in vesicle fusion is that fast and slow pools of vesicles result from differences in the distance between $\mathrm{Ca}^{2+}$ channels and vesicles (Neher, 1998). (C) Average minis and compound excitatory postsynaptic currents (cEPSC) from control and brp mutant Drosophila NMJs (Kittel et al., 2006b). The 10-to-90\% rise time (RT) is indicated with red lines (Control) and blue lines (brp). The lower panels show the average of 10 experiments each. (D) A kinetic model of how $\mathrm{Ca}^{2+}$ ions trigger vesicle fusion based on detailed studies of release at a large calyciform synapse within the brain stem (Schneggenburger and Neher, 2005). Figure taken from (Kittel et al., 2006a).

A functional consequence of mislocalised $\mathrm{Ca}^{2+}$ channels is illustrated in Figure $33 \mathrm{C}$. Whereas the rise time of minis was unaltered in brp mutants, the rise time of the compound excitatory junctional current was lengthened. This kinetic change appears to reflect an increased average distance between $\mathrm{Ca}^{2+}$ channels and vesicles (Fig. 33B). Similarly, the mislocalisation of $\mathrm{Ca}^{2+}$ channels may disrupt their proximity to $\mathrm{Ca}^{2+}$ activated $\mathrm{K}^{+}$channels, which could contribute to prolonged vesicle release. To investigate this possibility, pharmacological treatments could be used, in combination 
with deconvolution methods to estimate release rates of synaptic vesicles and to derive more quantitative descriptions of the quantal content, particularly during repetitively applied stimulation (Neher and Sakaba, 2001, 2003). In addition, the release machinery and its sensitivity to $\mathrm{Ca}^{2+}$ ions could be altered at brp mutant synapses. To clarify the latter, detailed kinetic studies of release and reaction schemes as depicted in Figure 33D will have to be performed (Schneggenburger and Neher, 2005). 


\subsubsection{Localisation of Bruchpilot within the active zone}

The presence of Bruchpilot appears to be a prerequisite for the formation of T-bars. Based on the STED images, it is conceivable that a ring of BRP tightly surrounds these dense aggregations, contained within the unlabelled centre of donuts (Fig. 10, 34B). BRP may establish a matrix that could act as a constraining guardian or gatekeeper for the active zone (Atwood, 2006), and may consist of fine filaments of Bruchpilot protein on the cytoplasmic surface of the presynaptic membrane (Fig. 34A).

Alternatively, Bruchpilot may itself be part of the T-bar structure. The N-terminal sequence of BRP shows homology to the vertebrate active zone component CAST, which has been implicated in the molecular organisation of active zones and colocalises with presynaptic $\mathrm{Ca}^{2+}$ channels (tom Dieck et al., 2005). At approximately $200 \mathrm{kDa}, \mathrm{BRP}$ is a large protein and although the coiled-coil domain-rich C-teminus $(\sim 110 \mathrm{kDa})$ is not conserved in the shorter CAST family proteins, this region exhibits a significant similarity to large cytoskeletal proteins such as myosin heavy chain. There have been descriptions of sequences of myosin heavy chain of similar size to the $\mathrm{C}$ terminal half of BRP forming rod-like structures extending over $150 \mathrm{~nm}$ or more (Slayter and Lowey, 1967). Bruchpilot may thus assemble in a polarised orientation with the C-terminal end protruding into the cytoplasm (Fig. 34F). As the Nc82 antibody epitope maps to the C-terminal half of BRP [S. Mertel, unpublished; (Wagh et al., 2006)], it is possible that the Nc82 rings observed in STED images reflect the edges of the T-bar platform at a distance of around $70 \mathrm{~nm}$ from the presynaptic membrane. In support of this concept, $\mathrm{Nc} 82$ and $\mathrm{Ca}^{2+}$ channel labels do not overlap precisely (Fig. $34 \mathrm{C}, \mathrm{F}$ ), whereas the staining of a newly developed antibody against a more $\mathrm{N}$-terminal region of BRP moves closer to the $\mathrm{Ca}^{2+}$ channel label (Fig. 34D, E, F). 

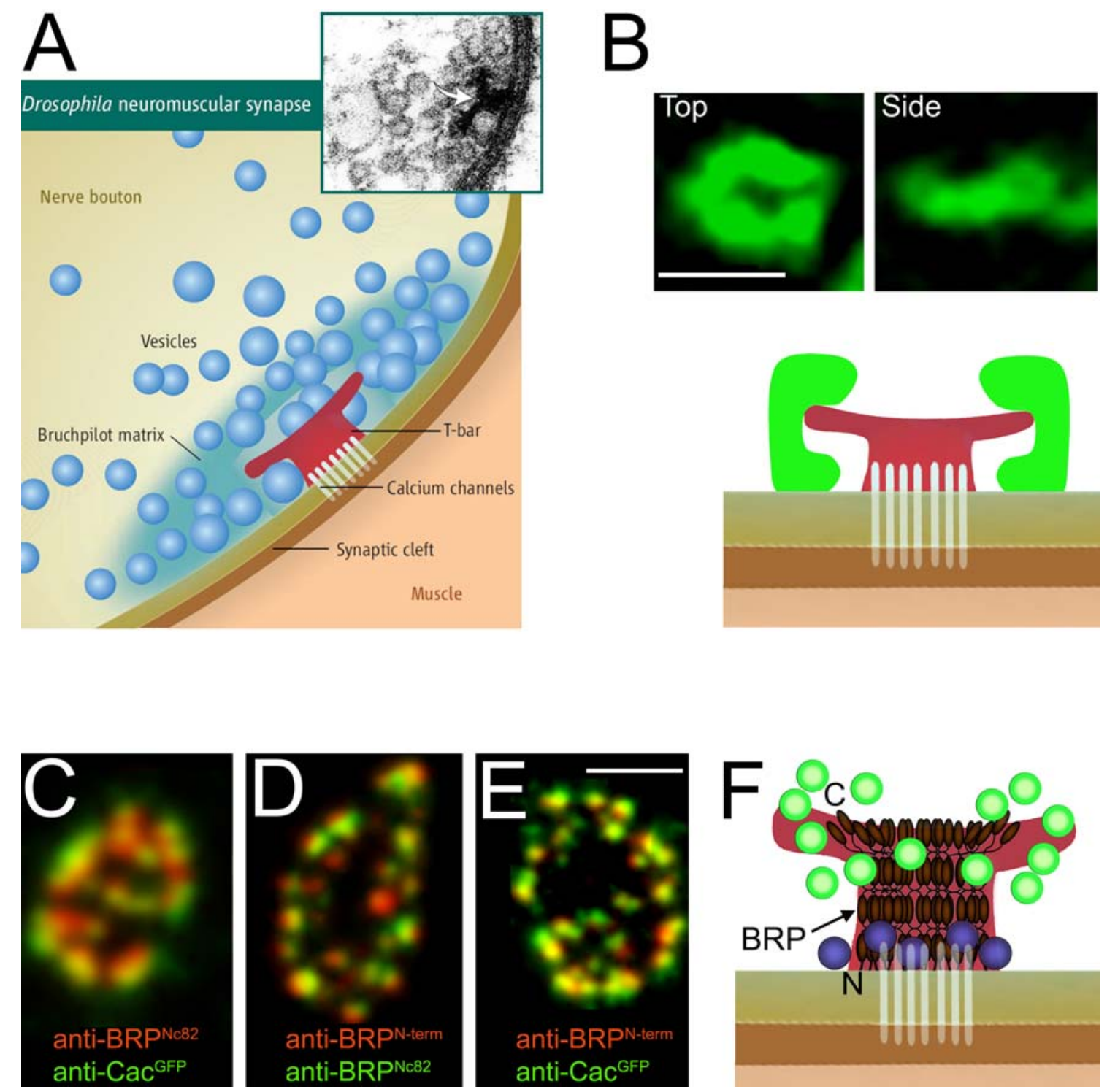

Figure 34. Localisation of Bruchpilot within the active zone.

(A) Transmission electron micrograph (top, right) illustrates assembly of synaptic vesicles at the T-bar in a Drosophila synapse. The synaptic vesicles are about $40 \mathrm{~nm}$ in diameter. The diagram illustrates the Drosophila T-bar surrounded by a donut-shaped zone of Bruchpilot protein, a cluster of $\mathrm{Ca}^{2+}$ channels, and several vesicles attracted to the active zone. The thickness, substructure, and lateral extent of the Bruchpilot matrix are not yet fully defined (Atwood, 2006). (B) Nc82 donuts viewed from above (left) and the side (right) with STED fluorescent microscopy. BRP may tightly embrace T-bars, located in the unlabelled centre of Nc82 rings. (C-E) Co-staining against two different BRP domains and $\mathrm{Ca}^{2+}$ channels at the larval NMJ. (C) The C-terminal Nc82 label (red) recognises a region of BRP that does not overlap precisely with GFP-tagged $\mathrm{Ca}^{2+}$ channels (stained against GFP; green). The Nc82 staining is located further towards the interior of the bouton. (D) The Nc82 label (green) does not completely overlap with the label of an antibody against an N-terminal region of BRP (red). (E) The staining pattern against the N-terminal epitope (red) co-localises well with the anti-GFP staining against GFPtagged $\mathrm{Ca}^{2+}$ channels (green). (F) Schematic diagram depicting BRP as part of the T-bar. The $\mathrm{N}$-terminal end is at the presynaptic membrane, in the vicinity of $\mathrm{Ca}^{2+}$ channels, whereas the $\mathrm{C}$ terminal end surrounds the edges of the T-bar platform. The green circles represent the Cterminal Nc82 label and the purple circles suggest regions of the N-terminal antibody label. (CE) Courtesy of S. Mertel, C. Wichmann, W. Fouquet. Scale bars, $250 \mathrm{~nm}$ in (B), $200 \mathrm{~nm}$ in (CE). 


\subsubsection{The function of T-bars}

Whether Bruchpilot is directly responsible for the clustering of $\mathrm{Ca}^{2+}$ channels, or whether it exerts this influence indirectly by enabling the formation of T-bars, remains to be elucidated. Based on our current state of knowledge, it is difficult to dissect the function of T-bars independent of defective $\mathrm{Ca}^{2+}$ channel clustering at the active zone. The structural arrangement of the T-bar has prompted suggestions that this presynaptic density participates in the tethering of synaptic vesicles to direct them to their optimal position for fast exocytosis at the base of the T-bar [Fig. 35A; (Koenig and Ikeda, 1996; Feeney et al., 1998; Prokop, 1999; Zhai and Bellen, 2004; Atwood, 2006; Prokop and Meinertzhagen, 2006)]. It is also possible that the T-bar itself is required for clustering $\mathrm{Ca}^{2+}$ channels at the active zone. In the larval flesh fly Sarcophaga bullata, structural synaptic parameters were found to strongly match those of the anatomically homologous muscles in Drosophila melanogaster (Feeney et al., 1998). Freeze fractures through the presynaptic membrane of Sarcophaga showed intra-membranous particles, thought to constitute membrane-associated $\mathrm{Ca}^{2+}$ channels (Fig. 35D). These particles radiated out in three prongs, resembling the three-pronged base of the Sarcophaga T-bar (Feeney et al., 1998). It is therefore conceivable, that the similarly multi-pronged T-bars at Drosophila neuromuscular synapses (Fig 35B, C) contain intracellular portions of $\mathrm{Ca}^{2+}$ channels and that clustering of the latter would not take place in the absence of $\mathrm{T}$ bars. Studies at the larval Drosophila NMJ have indicated a correlation between the number of T-bars and the strength of synaptic transmission (Jia et al., 1993; Stewart et al., 1996), and at the crayfish NMJ, the activity-induced addition of presynaptic dense bodies has been proposed to elevate vesicle release probability (Wojtowicz et al., 1994). The work presented here supports this hypothesis and suggests an involvement of Bruchpilot or related factors in synaptic plasticity by promoting the clustering of $\mathrm{Ca}^{2+}$ channels at the active zone membrane. 

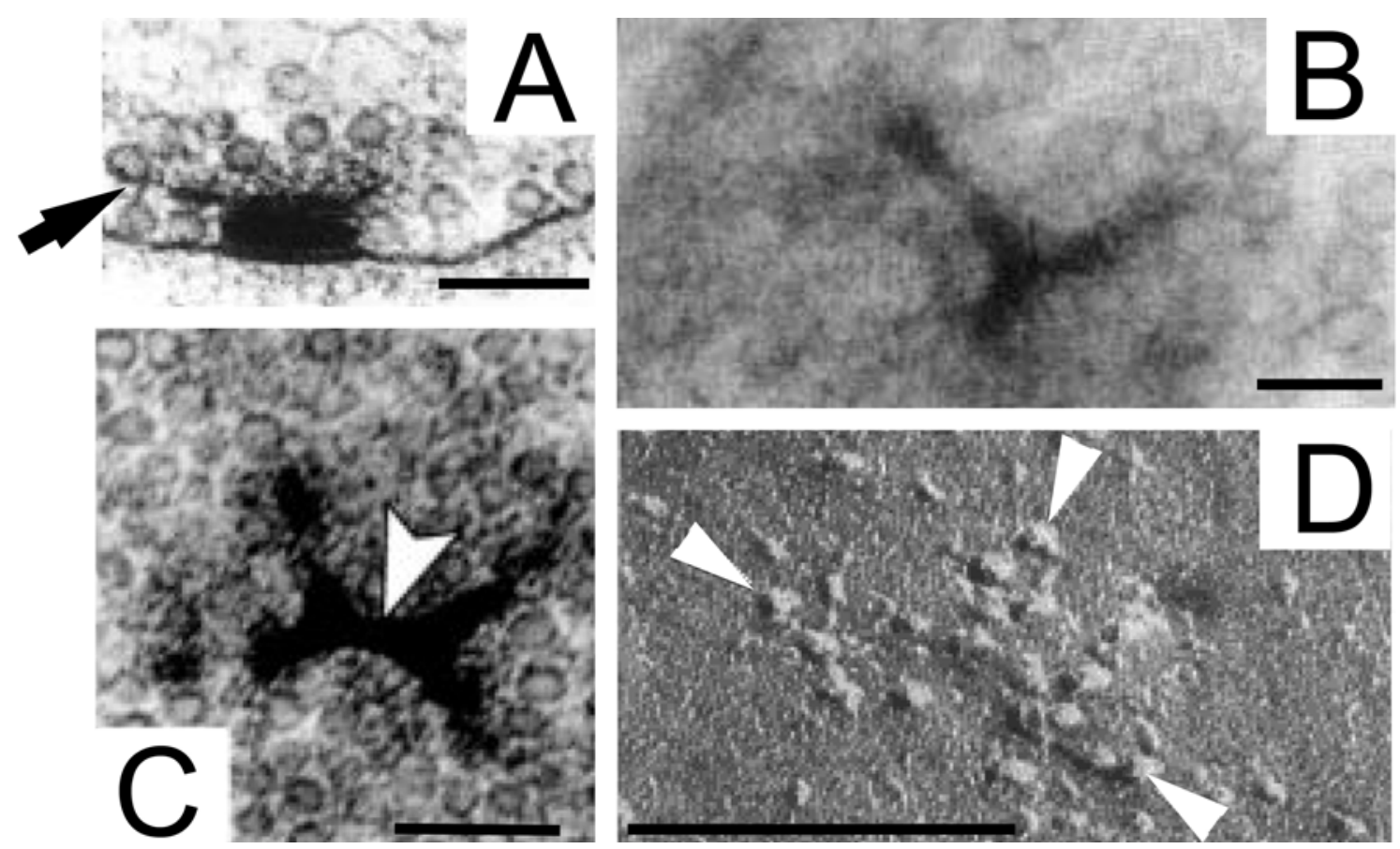

Figure 35. Association of T-bars with synaptic vesicles and putative $\mathrm{Ca}^{2+}$ channels.

(A-C) Thin sections of T-bars viewed with conventional transmission electron microscopy. (A) Side view (transverse section through a synaptic contact) of a T-bar from a Drosophila embryo (Prokop, 1999) shows thin filaments (arrow) which may connect, or tether synaptic vesicles to the T-bar. (B, C) Top view (oblique section through a T-bar) of a larval Drosophila T-bar shows the three- [B; (Atwood et al., 1993)] or four-pronged [C, arrowhead; (Prokop, 1999)] nature of the column and/or platform of the T-bar. (D) Freeze-fracture replica showing an active zone of Sarcophaga as an aggregation of large intra-membranous particles, possibly $\mathrm{Ca}^{2+}$ channels, radiating out in three arms (arrowheads) on the axolemmal P-face (Feeney et al., 1998). Scale bars $125 \mathrm{~nm}$ in (A), $100 \mathrm{~nm}$ in (B, D), and $150 \mathrm{~nm}$ in (C).

The large scaffolding protein Bassoon has been associated with the structural assembly of active zones at vertebrate synapses (Khimich et al., 2005; tom Dieck et al., 2005). In sensory inner hair cells of mice mutant for Bassoon, the large electron-dense ribbons (see Chapter 2.2.2.), normally found anchored to active zones, were detached from the presynaptic membrane and observed floating in the cytoplasm. This defect was accompanied by a decrease in the number of readily-releasable vesicles and possibly impaired $\mathrm{Ca}^{2+}$ influx, though the fundamental process of exocytosis was preserved (Khimich et al., 2005). As no floating electron-dense structures were detected in BRP mutants, Bruchpilot appears to be required for the formation, rather than the anchoring of T-bars. This could be interpreted as an indication that Bruchpilot is an integral component of the T-bar. The data presented in this thesis demonstrate that synapses lacking T-bars are in principle still functional. Furthermore, as illustrated by the initial facilitation of brp mutant currents during tetanic stimulation (Fig. 20A), vesicle 
availability did not appear rate-limiting under low frequency stimulation. The decrease in readily-releasable vesicles observed at sensory synapses of Bassoon mutants probably reflects the physiological demands of this synaptic system to sustain continuous exocytosis. Correspondingly, sensory ribbons may perform a more prominent role in delivering vesicles to release sites, than the T-bars at the Drosophila NMJ, where short bursts of exocytosis occur. In brp mutants, a more subtle defect in the tethering and presentation of synaptic vesicles may be disguised by the prominent phenotype following defective clustering of $\mathrm{Ca}^{2+}$ channels. However, an impaired delivery of vesicles may be reflected by the slightly reduced number of vesicles docked to the active zone membrane in brp mutants (see Chapter 4.1.3.). The Drosophila genome apparently encodes no Bassoon homologue. Considering the N-terminal homology of BRP to mammalian ELKS/CAST/ERC proteins which interact with Bassoon (see Chapters 2.2.2. and 4.1.1.), and the similarity of the C-terminal half of BRP to large structural proteins, Bruchpilot may instead incorporate functions of several vertebrate proteins in a single polypeptide.

An additional function of ribbons at sensory synapses may be the presentation of vSNAREs of vesicles within a 'striking distance' of t-SNAREs on the plasma membrane (Zenisek et al., 2000). This could ensure a high vesicle release probability following the opening of $\mathrm{Ca}^{2+}$ channels. Similarly, the ribs of the cytomatrix at the frog neuromuscular active zone (see Chapter 2.2.2.) have been suggested to promote the association of $\mathrm{v}$ - and t-SNARES with $\mathrm{Ca}^{2+}$ channels (Harlow et al., 2001). Such mechanisms may also take place at Drosophila active zones, and the association of Bruchpilot with both $\mathrm{Ca}^{2+}$ channels and T-bars strengthens this concept and presents a candidate molecule that may provide the missing link.

A further scenario is that T-bars, together with their associated $\mathrm{Ca}^{2+}$ channels, may participate in a specific pathway of endocytosis. In Drosophila photoreceptor terminals, vesicles have been distinguished that bud off either below the T-bar or perisynaptically (Koenig and Ikeda, 1996). In contrast to the perisynaptic reformation, vesicles at the Tbar form more rapidly and can be blocked by high extracellular $\mathrm{Mg}^{2+}$ or low $\mathrm{Ca}^{2+}$. A rapid form of membrane retrieval may also occur near ribbons (Lenzi and von Gersdorff, 2001), and increased $\mathrm{Ca}^{2+}$ influx has been reported to accelerate endocytosis (Klingauf et al., 1998; Wu et al., 2005). In this context, the intermediate rufflings or 
invaginations of the presynaptic membrane in brp mutants (Fig. 16D) may represent defective or stalled processes of vesicle reformation.

\subsection{4 $\underline{\text { Outlook }}$}

Several fundamental questions remain open. Is the heterogeneous rate of vesicle fusion upon $\mathrm{Ca}^{2+}$ influx a general phenomenon or only found at specialized synapses? How do Bruchpilot and its homologues exert their involvement in the organization of the active zone? Does Bruchpilot directly interact with $\mathrm{Ca}^{2+}$ channels and is it part of the T-bar? Can $\mathrm{Ca}^{2+}$ channel clustering occur in the absence of T-bars, and how dynamic is the molecular composition of active zones? The recent identification of key active zone molecules now allows these questions to be addressed. 


\subsection{Drosophila glutamate receptor subunits GluRIIA and GluRIIB}

\subsubsection{Biophysical properties}

An electrophysiological analysis was carried out to examine the differential effects of the two glutamate receptor subunits GluRIIA and GluRIIB on synaptic strength at the developing Drosophila NMJ. The presence of a mid-C-terminal GFP-tag on either subunit had no discernable effect on the synaptic physiology. Therefore, both transgenically labelled subunits can be considered as suitable tools for visualising the fate of identified receptor fields during the development of synaptic contacts in vivo (Rasse et al., 2005). However, the incorporation of either GluRIIA or GluRIIB into receptor complexes strongly affected synaptic efficacy. In line with previous reports (DiAntonio et al., 1999; Sigrist et al., 2002), GluRIIA rescued synapses had a high postsynaptic sensitivity. Considering the small average amplitude of minis in GluRIIB rescues and the intermediate amplitude of wild-type minis, this conclusion can be extended to describe a correlation between the level of GluRIIA expression in receptor fields and the quantal size. This effect may in part be attributed to the enlarged receptor fields of GluRIIA rescues relative to GluRIIB rescues, and possibly also to an increased density of receptor complexes.

Biophysical properties have also been reported to underlie the differential influences of these two subunits on postsynaptic sensitivity (DiAntonio et al., 1999). Outside-out patches from larval muscles demonstrated that extrasynaptic GluRIIB channels desensitise more rapidly than those of GluRIIA. Alterations in the time course of desensitisation have also been described for vertebrate glutamate receptors differing in subunit composition, in editing of the $\mathrm{R} / \mathrm{G}$ site, and in terms of flip-flop splice variants (Lomeli et al., 1994; Mosbacher et al., 1994). Correspondingly, the long decay kinetics of GluRIIA and gluRIIA hypo larval synaptic currents may reflect these desensitisation properties of ion channels. However, manifesting this suggestion will require further analysis of factors influencing the time course of compound synaptic currents in this system (Jonas and Spruston, 1994; Pawlu et al., 2004). The smaller quantal size of GluRIIB rescues may be influenced by the rapid desensitisation kinetics of their ion channels. Drosophila glutamate receptors can desensitise before opening (Heckmann and Dudel, 1997), which suggests that an increased rate of desensitisation could lead to a decrease in the synaptic current. As the synaptic currents are much shorter than the 
muscle membrane time constant (Jan and Jan, 1976a), the rapid currents of GluRIIB receptors would cause less synaptic depolarisation than GluRIIA currents.

Furthermore, DiAntonio et al. found no difference in the single channel conductance of extrasynaptic larval GluRIIA and GluRIIB receptors. In contrast, the unitary conductance of synaptic receptors was reportedly increased in embryos expressing reduced levels of GluRIIB (Chen and Featherstone, 2005). These latter estimations were made by measuring the amplitude of individual steps in the decaying phase of minis, and the reduction of GluRIIB expression followed postsynaptic loss of the PDZdomain-protein Dlg. The discrepancy between these two reports could be explained by different biophysical properties of larval and embryonic receptors, or synaptic versus extrasynaptic receptors. To clarify this issue, the single channel conductance of synaptic receptors was estimated in GluRIIA and GluRIIB rescued embryos by adopting the approach of Chen and Featherstone. The results presented here, describe a very similar unitary conductance of GluRIIA and GluRIIB containing receptors ( $\sim 108 \mathrm{pS}$ and $\sim 111$ pS), which corresponds well to the values obtained by DiAntonio et al. from extrasynaptic larval receptors (both $\sim 120 \mathrm{pS}$ ) and wild-type embryonic receptors $[\sim 115$ pS; (Nishikawa and Kidokoro, 1995; Chen and Featherstone, 2005)]. The heterogeneous unitary conductance observed by Chen and Featherstone may therefore reflect a specific influence of Dlg, rather than GluRIIA or GluRIIB, on receptor ion channel conductance. Whether such a modulation by Dlg is subunit-specific will require further analysis.

\subsubsection{Implications for synaptic function}

It is conceivable that the Drosophila neuromuscular system exploits these different properties of glutamate receptor subunits to influence synaptic strength. Correspondingly, a differential subunit expression has been documented to occur throughout the development of the NMJ. During embryogenesis, GluRIIB is initially expressed and then gradually replaced by GluRIIA (Petersen et al., 1997). Similarly, novel receptor fields grow in larvae by continuously switching between the incorporation of GluRIIB and GluRIIA. Interestingly, the genetic elimination of transmitter release leads to a recruitment of GluRIIA into receptor fileds. This may reflect a postsynaptic attempt to maximise signal transmission (A. Schmid, personal 
communication). Such a subunit-specific recruitment may be regulated by second messengers, acting to balance pre- and postsynaptic strength (Smart, 1997; Davis et al., 1998). The hypo-innervation of Drosophila muscles leads to a compensatory increase in quantal size (Davis and Goodman, 1998), which has been suggested to be mediated by the selective recruitment of GluRIIA (DiAntonio et al., 1999). Correspondingly, bruchpilot mutants had elevated mini amplitudes and enlarged receptor fields, which could be interpreted as a postsynaptic compensation for the presynaptic release defect. Whether these receptor fields contain a large fraction of GluRIIA will require further investigation. Conversely, decreases in quantal size appear to be compensated by the presynapse in terms of quantal content (Petersen et al., 1997). The results presented here, describe this compensation for gluRIIA hypo larvae, suffering from minimal glutamate receptor expression. Such a response requires homeostatic retrograde signalling (Petersen et al., 1997; DiAntonio et al., 1999), and a potential pathway relies on the activation of glutamate receptors, the influx of $\mathrm{Ca}^{2+}$ ions and postsynaptic depolarisation (Paradis et al., 2001; Yoshihara et al., 2005). The association of GluRIIA with the morphological outgrowth of the neuronal innervation [see Chapter 2.5.3.; (Sigrist et al., 2000; Sigrist et al., 2002)] suggests a specific role for this subunit in such a pathway. Most intriguingly, however, the increase of presynaptic T-bars following an elevated expression of GluRIIA (Sigrist et al., 2002) presents Bruchpilot as a potential target of a postsynaptic control over presynaptic strength. It will be of great interest to examine this possibility in future studies 


\subsection{Drosophila Basigin}

The data presented in this thesis identify the small transmembrane Ig CAM protein Basigin as a novel component of periactive zones at the Drosophila NMJ. Bsg is involved in the formation and growth of synaptic boutons and in a similar way to other Ig CAM molecules, such as Drosophila Fas II, Bsg may exert this control by mediating the adhesion between pre- and postsynaptic membranes. Furthermore, Bsg plays an essential role in regulating the compartmentalisation within the presynaptic terminal. Basigin ensures the adequate cortical clustering of synaptic vesicles and inhibits asynchronous release, possibly by organising the presynaptic actin-based cytoskeleton.

\subsubsection{Involvement of Basigin in vesicle mobilisation}

Synaptic vesicles are not scattered randomly throughout the presynaptic compartment, but rather accumulate in cortical regions and in the vicinity of the active zone plasma membrane. Correspondingly, electron micrographs of axon terminals at the Drosophila $\mathrm{NMJ}$ show that the central regions are largely devoid of synaptic vesicles [Fig. 4C, 12C, D, 16A, C; (Atwood et al., 1993)]. Although attempts have been made to identify functionally distinct vesicle pools (see Chapter 2.2.3.), these do not appear to be morphologically segregated into distinct clusters, and vesicle populations of the recycling and reserve pools may even be intermixed (Rizzoli and Betz, 2005). Such a sub-cellular organisation would require the precise and specific mobilisation and trafficking of vesicles with similar properties.

In bsg mutant larvae, a dispersion of synaptic vesicles throughout axon terminals was observed, and the dynamic labelling of cycling vesicles uncovered aberrant trafficking to inner regions of boutons. Under these conditions, synaptic vesicle recycling is normally restricted to the bouton periphery. Only during excessive stimulation combined with a genetic manipulation of endocytosis, have more central regions previously been labelled and subsequently assigned to reserve pool of vesicles (Kuromi and Kidokoro, 1998, 2003). Furthermore, bsg mutants exhibited excessive, asynchronous vesicle release. The populations of rapidly and slowly releasing vesicles (see Chapters 5.1.1. and 2.2.3.) have been linked to the synchronous and asynchronous 
component of action potential-triggered vesicle release, respectively (Sakaba, 2006). The absence, or decrease in the number of rapidly releasing vesicles at bruchpilot mutant synapses (see Chapters 4.1.3. and 5.1.1.) had no effect on the frequency of minis, and supports the suggestion that spontaneous fusion events may arise from the population of slowly releasing vesicles (Trommershäuser et al., 2003). In agreement with this concept, the increased asynchronous component of evoked release in basigin mutants correlates with an elevated frequency of spontaneous release, and could be interpreted as an aberrant mobilisation of slowly releasing vesicles, not tightly associated with $\mathrm{Ca}^{2+}$ channels.

Whether the vesicles contributing to this release phenotype at bsg mutant synapses arise from the reserve pool will require further investigation. It should however also be noted, that appointing vesicles to a specific population such as the reserve pool may be inadequate when the mobilisation and trafficking of vesicles is impaired, and distinct functional identities of synaptic vesicles may be less clearly assigned. An elevated contribution of asynchronous release has been reported to occur naturally at particular synapses of the mammalian CNS, and is thought to reflect longer $\mathrm{Ca}^{2+}$ transients and a large distance between the $\mathrm{Ca}^{2+}$ source and vesicles (Hefft and Jonas, 2005). This supports the notion that the asynchronous component of release in basigin mutants is mediated by vesicles not located in an optimal position within the active zone. But similarly, altered $\mathrm{Ca}^{2+}$ signalling, including the release of $\mathrm{Ca}^{2+}$ ions from intra-cellular stores, may contribute to excessive exocytosis, and will have to be addressed by future studies.

The successful rescue of $b s g$ mutant defects by presynaptic re-expression of wild-type Bsg, implies the specific requirement for Bsg at the presynaptic membrane to restrict the number of release-competent synaptic vesicles. Which particular steps of synaptic vesicle recruitment and/or exocytosis are regulated by Bsg remains to be elucidated. Notably, the accumulation of plasma membrane markers within the interior of bsg mutant boutons suggests sorting defects between plasma membrane and inner endocytotic compartments. 


\subsubsection{Association of Basigin with the actin cytoskeleton}

The aberrant spatial distribution, recycling, and release properties of synaptic vesicles in bsg mutant terminals appear to be associated with an altered organisation of the presynaptic actin cytoskeleton. This is of particular interest in view of the widelyrecognised notion that actin is involved in the structural organisation of synaptic terminals and vesicle cycling (Doussau and Augustine, 2000). Actin filaments have been observed to surround vesicle clusters in nerve terminals of various neurons. The actin network has therefore been proposed to provide a physical barrier to impede vesicle dispersion and to regulate the availability of the reserve pool. However, as the actin cytoskeleton is involved in different steps of synaptogenesis and synaptic maturation, it has remained challenging to address its specific role in the organisation and mobilisation of synaptic vesicles. Such approaches have largely relied on pharmacological treatments to disrupt the actin network, and have produced a wide spectrum of results, depending on criteria such as the type of synaptic contact, the age of the synapse, and probably also the differential stability of distinct actin filaments (Dillon and Goda, 2005). Interestingly, application of the G-actin-sequestering drug Latrunculin A has been shown to increase vesicle release probability in cultured hippocampal neurons, which adds support to the concept that actin could participate in the retention of synaptic vesicles (Morales et al., 2000). In agreement with this report, Basigin function is required for the organisation of the presynaptic actin cytoskeleton, the spatial distribution of synaptic vesicles, and the negative regulation of neurotransmitter release. These results therefore establish a strong link between the integrity of presynaptic actin filaments and the accuracy of synaptic transmission.

Considering the decrease in number and increase in size of bsg mutant boutons, it is conceivable that Basigin promotes the expansion of the $\mathrm{NMJ}$ via the addition of new synaptic terminals through modulations of the actin network. Basigin activity at the presynaptic membrane may recruit Spectrin or other actin-associated proteins to directly participate in the organisation of the sub-cortical actin-rich cytoskeleton underlying the plasma membrane. Previous work has suggested that the postsynaptic membrane and associated cytoskeleton surrounding synaptic boutons may impose a barrier to presynaptic outgrowth. Thus, a coordinated regulation of both the pre- and postsynaptic cytoskeleton may be essential for the adequate growth and division of synaptic 
terminals (Ruiz-Canada et al., 2004). The normal growth and budding of boutons requires Basigin function in both synaptic compartments. Similar to Fas II (Packard et al., 2003), Basigin may therefore act as a cell-adhesion molecule in a trans-synaptic system by regulating the degree of association between pre- and postsynaptic membranes, and thereby exerting control over the development of synaptic contacts. 


\section{Abbreviations}

\begin{tabular}{|c|c|}
\hline $\mathrm{ABP}$ & AMPA receptor-binding protein \\
\hline AEL & after egg-laying \\
\hline AMPA & $\alpha$-amino-3-hydroxyl-5-methyl-4-isoxalone propionic acid \\
\hline AP & action potential \\
\hline a.u. & arbitrary units of intensity \\
\hline BRP & Bruchpilot \\
\hline Bsg & Basigin \\
\hline $\mathrm{Cac}$ & Cacophony \\
\hline CAST & cytomatrix at the active zone-associated structural protein \\
\hline CAZ & cytomatrix at the active zone \\
\hline $\mathrm{C}_{\mathrm{m}}$ & membrane capacitance \\
\hline CNS & central nervous system \\
\hline $\mathrm{Dlg}$ & Drosophila PSD-95/SAP90 orthologue Discs-large \\
\hline eag & ether a go-go \\
\hline EGTA & Ethyleneglycol-bis( $\beta$-aminoethyl $)-\mathrm{N}, \mathrm{N}, \mathrm{N}^{\prime}, \mathrm{N}^{\prime}$-tetraacetic Acid \\
\hline eEJC & evoked excitatory junctional current \\
\hline ERC & ELKS/Rab6-interacting protein/CAST \\
\hline Fas II & Drosophila NCAM homologue Fasciclin II \\
\hline FE & focal electrode \\
\hline GABA & $\gamma$-aminobutyric acid \\
\hline GFP & green fluorescent protein \\
\hline GRIP & glutamate receptor interacting protein \\
\hline Ig-CAM & cell adhesion molecule of the immunoglobulin superfamily \\
\hline $\mathrm{K}_{\mathrm{Ca}}$ channel & calcium-activated potassium channel \\
\hline LTD & long-term depression \\
\hline LTP & long-term potentiation \\
\hline MAB & monoclonal antibody \\
\hline $\mathrm{ME}$ & microelectrode \\
\hline mEJC & miniature excitatory junctional current \\
\hline Mini & mEJC \\
\hline Munc13 & mammalian homologue of Unc13 \\
\hline NCAM & neuronal cell adhesion molecule \\
\hline
\end{tabular}




$\begin{array}{ll}\text { NMDA } & \text { N-methyl-D-aspartate } \\ \text { NMJ } & \text { neuromuscular junction } \\ \text { PICK1 } & \text { protein interacting with C kinase 1 } \\ \text { PSD } & \text { postsynaptic density } \\ \text { R }_{\mathrm{s}} & \text { series resistance } \\ \text { Rim1 } & \text { Rab3-interacting molecule-1 } \\ \mathrm{R}_{\mathrm{m}} & \text { membrane resistance } \\ \text { RNAi } & \text { RNA interference } \\ \text { SAP-97 } & \text { synapse-associated protein } \\ \text { SEVC } & \text { single-electrode voltage clamp } \\ \text { SE } & \text { suction electrode } \\ \text { Sh } & \text { shaker } \\ \text { SNAP } & \text { soluble } N \text {-ethylmaleimide-sensitive factor attachment protein } \\ \text { SNARE } & \text { SNAP receptor } \\ \text { SSR } & \text { subsynaptic reticulum } \\ \text { STED } & \text { stimulated emission depletion } \\ \text { Syn } & \text { Synapsin } \\ \text { Syt } & \text { Synaptotagmin } \\ \text { TEVC } & \text { two-electrode voltage clamp } \\ \text { TARP } & \text { transmembrane AMPA receptor regulatory protein } \\ \text { Unc13 } & \text { uncoordinated protein-13 } \\ \text { UTR } & \text { untranslated region } \\ \mathrm{V}_{\text {cmd }} & \text { command potential } \\ \text { VLM } & \text { ventral longitudinal muscle } \\ V_{m} & \text { membrane potential } \\ & \end{array}$

Proteins are written in regular font beginning with an upper case letter, whereas genes are written in italic lower case letters. 


\section{Table of Figures}

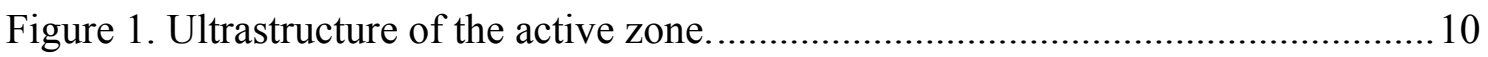

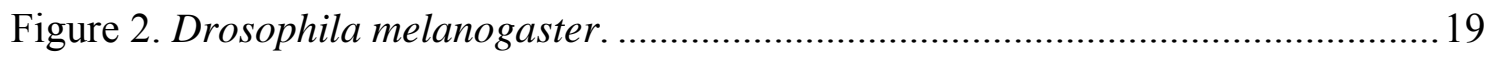

Figure 3. Larval Drosophila neuromusculature.................................................20

Figure 4. The structure of synaptic connections at the larval NMJ. ............................22

Figure 5. The two-electrode voltage clamp in operation at the larval NMJ. ..................31

Figure 6. Example traces of two-electrode voltage clamp recordings.......................... 32

Figure 7. Embryonic preparation for patch-clamp recordings................................... 34

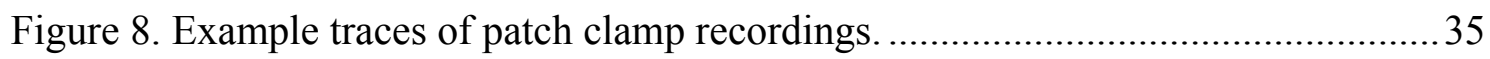

Figure 9. MAB Nc82 labels a component of the presynaptic active zone.....................40

Figure 10. Nc82 recognises donut-shaped structures........................................... 41

Figure 11. Drosophila Bruchpilot shows N-terminal homology to ELKS/CAST/ERK. 42

Figure 12. Defective active zone structure following suppression of Bruchpilot..........43

Figure 13. Suppresion of Bruchpilot impairs neurotransmitter release. ........................ 44

Figure 14. Mutants of bruchpilot lack the neuromuscular Nc82 label. .........................46

Figure 15. Junctional morphology of bruchpilot mutants....................................... 47

Figure 16. Complete loss of T-bars from bruchpilot mutant neuromuscular synapses. . 48

Figure 17. Electrophysiological characterisation of bruchpilot mutant NMJs. ..............49

Figure 18. Distribution of exo- and endocytotic proteins at bruchpilot mutant NMJs...50

Figure 19. Altered kinetic properties of evoked responses in bruchpilot mutants..........51

Figure 20. Changes in short-term plasticity at bruchpilot mutant NMJs.......................52

Figure 21. Increased sensitivity to the calcium buffer EGTA in bruchpilot mutants.....53

Figure 22. Mislocalisation of presynaptic calcium channels in bruchpilot mutants.......54

Figure 23. Expression of fluorescently tagged receptor subunits at the NMJ. ...............56

Figure 24. Effects of the glutamate receptor subunit composition on synaptic efficacy.57 
Figure 25. Receptor subunit composition determines quantal size. 58

Figure 26. Minimal glutamate receptor expression leads to presynaptic compensation. 60

Figure 27. Basigin accumulation at the NMJ 62

Figure 28. Diffuse distribution of synaptic vesicles in basigin mutant boutons. 63

Figure 29. Altered recycling pathway of vesicles at basigin mutant synapses. 65

Figure 30. Presynaptic loss of Basigin elevates the frequency of spontaneous release..66

Figure 31. Excessive asynchronous evoked transmitter release in basigin mutants. 67

Figure 32. The distribution of cytoskeletal markers is disrupted in basigin mutants. ....69

Figure 33. Vesicle pools and release kinetics. 72

Figure 34. Localisation of Bruchpilot within the active zone. 75

Figure 35. Association of T-bars with synaptic vesicles and putative $\mathrm{Ca}^{2+}$ channels..... 77 


\section{References}

Adler EM, Augustine GJ, Duffy SN, Charlton MP (1991) Alien intracellular calcium chelators attenuate neurotransmitter release at the squid giant synapse. $\mathrm{J}$ Neurosci 11:1496-1507.

Altrock WD, tom Dieck S, Sokolov M, Meyer AC, Sigler A, Brakebusch C, Fassler R, Richter K, Boeckers TM, Potschka H, Brandt C, Loscher W, Grimberg D, Dresbach T, Hempelmann A, Hassan H, Balschun D, Frey JU, Brandstatter JH, Garner CC, Rosenmund C, Gundelfinger ED (2003) Functional inactivation of a fraction of excitatory synapses in mice deficient for the active zone protein bassoon. Neuron 37:787-800.

Atwood HL (1967) Variation in physiological properties of crustacean motor synapses. Nature 215:57-58.

Atwood HL (2006) Neuroscience. Gatekeeper at the synapse. Science 312:1008-1009.

Atwood HL, Wojtowicz JM (1999) Silent synapses in neural plasticity: current evidence. Learn Mem 6:542-571.

Atwood HL, Karunanithi S (2002) Diversification of synaptic strength: presynaptic elements. Nat Rev Neurosci 3:497-516.

Atwood HL, Govind CK, Wu CF (1993) Differential ultrastructure of synaptic terminals on ventral longitudinal abdominal muscles in Drosophila larvae. J Neurobiol 24:1008-1024.

Augustin I, Rosenmund C, Sudhof TC, Brose N (1999) Munc13-1 is essential for fusion competence of glutamatergic synaptic vesicles. Nature 400:457-461.

Augustine GJ, Neher E (1992) Neuronal Ca2+ signalling takes the local route. Curr Opin Neurobiol 2:302-307.

Axoclamp-2B (1997) Microelectrode Clamp Theory and Operation. Union City, CA: Axon Instruments, Inc.

Barrett EF, Stevens CF (1972) The kinetics of transmitter release at the frog neuromuscular junction. J Physiol 227:691-708.

Barry MF, Ziff EB (2002) Receptor trafficking and the plasticity of excitatory synapses. Curr Opin Neurobiol 12:279-286.

Bate M (1990) The embryonic development of larval muscles in Drosophila. Development 110:791-804.

Bennett MR, Farnell L, Gibson WG (2000) The probability of quantal secretion near a single calcium channel of an active zone. Biophys J 78:2201-2221.

Besse F, Mertel S, Kittel RJ, Wichmann C, Rasse TM, Sigrist SJ, Ephrussi A (2006) The Ig CAM Basigin controls the distribution and release of synaptic vesicles at Drosophila neuromuscular junctions. submitted.

Betz A, Thakur P, Junge HJ, Ashery U, Rhee JS, Scheuss V, Rosenmund C, Rettig J, Brose N (2001) Functional interaction of the active zone proteins Munc13-1 and RIM1 in synaptic vesicle priming. Neuron 30:183-196.

Beumer K, Matthies HJ, Bradshaw A, Broadie K (2002) Integrins regulate DLG/FAS2 via a CaM kinase II-dependent pathway to mediate synapse elaboration and stabilization during postembryonic development. Development 129:3381-3391.

Beumer KJ, Rohrbough J, Prokop A, Broadie K (1999) A role for PS integrins in morphological growth and synaptic function at the postembryonic neuromuscular junction of Drosophila. Development 126:5833-5846.

Bliss TV, Lømo T (1973) Long-lasting potentiation of synaptic transmission in the dentate area of the anaesthetized rabbit following stimulation of the perforant path. J Physiol 232:331-356. 
Bliss TV, Collingridge GL (1993) A synaptic model of memory: long-term potentiation in the hippocampus. Nature 361:31-39.

Borgdorff AJ, Choquet D (2002) Regulation of AMPA receptor lateral movements. Nature 417:649-653.

Braithwaite SP, Meyer G, Henley JM (2000) Interactions between AMPA receptors and intracellular proteins. Neuropharmacology 39:919-930.

Brand AH, Perrimon N (1993) Targeted gene expression as a means of altering cell fates and generating dominant phenotypes. Development 118:401-415.

Broadie KS (2000) Electrophysiological Approaches to the Neuromusculature. Cold Spring Harbor, New York: Cold Spring Harbor Laboratory Press.

Broadie KS, Bate M (1993a) Development of the embryonic neuromuscular synapse of Drosophila melanogaster. J Neurosci 13:144-166.

Broadie KS, Bate M (1993b) Development of larval muscle properties in the embryonic myotubes of Drosophila melanogaster. J Neurosci 13:167-180.

Buchner E, Buchner S, Crawford G, Mason WT, Salvaterra PM, Sattelle DB (1986) Choline acetyltransferase-like-immunoreactivity in the brain of Drosophila melanogaster. Cell Tissue Res 246:57-62.

Buchner E, Bader R, Buchner S, Cox J, Emson PC, Flory E, Heizmann CW, Hemm S, Hofbauer A, Oertel WH (1988) Cell-specific immuno-probes for the brain of normal and mutant Drosophila melanogaster. I. Wildtype visual system. Cell Tissue Res 253:357-370.

Budnik V (1996) Synapse maturation and structural plasticity at Drosophila neuromuscular junctions. Curr Opin Neurobiol 6:858-867.

Budnik V, Zhong Y, Wu CF (1990) Morphological plasticity of motor axons in Drosophila mutants with altered excitability. J Neurosci 10:3754-3768.

Burns ME, Augustine GJ (1995) Synaptic structure and function: dynamic organization yields architectural precision. Cell 83:187-194.

Cao YQ, Piedras-Renteria ES, Smith GB, Chen G, Harata NC, Tsien RW (2004) Presynaptic $\mathrm{Ca}^{2+}$ channels compete for channel type-preferring slots in altered neurotransmission arising from $\mathrm{Ca}^{2+}$ channelopathy. Neuron 43:387-400.

Casadio A, Martin KC, Giustetto M, Zhu H, Chen M, Bartsch D, Bailey CH, Kandel ER (1999) A transient, neuron-wide form of CREB-mediated long-term facilitation can be stabilized at specific synapses by local protein synthesis. Cell 99:221237.

Chad JE, Eckert R (1984) Calcium domains associated with individual channels can account for anomalous voltage relations of CA-dependent responses. Biophys $\mathrm{J}$ 45:993-999.

Chen K, Featherstone DE (2005) Discs-large (DLG) is clustered by presynaptic innervation and regulates postsynaptic glutamate receptor subunit composition in Drosophila. BMC Biol 3:1.

Chen L, Chetkovich DM, Petralia RS, Sweeney NT, Kawasaki Y, Wenthold RJ, Bredt DS, Nicoll RA (2000) Stargazin regulates synaptic targeting of AMPA receptors by two distinct mechanisms. Nature 408:936-943.

Collingridge GL, Isaac JT, Wang YT (2004) Receptor trafficking and synaptic plasticity. Nat Rev Neurosci 5:952-962.

Couteaux R, Pecot-Dechavassine M (1970) [Synaptic vesicles and pouches at the level of "active zones" of the neuromuscular junction]. C R Acad Sci Hebd Seances Acad Sci D 271:2346-2349.

Craven SE, Bredt DS (1998) PDZ proteins organize synaptic signaling pathways. Cell 93:495-498. 
Crossin KL, Krushel LA (2000) Cellular signaling by neural cell adhesion molecules of the immunoglobulin superfamily. Dev Dyn 218:260-279.

Cull-Candy SG, Parker I (1982) Rapid kinetics of single glutamate-receptor channels. Nature 295:410-412.

Curtin KD, Meinertzhagen IA, Wyman RJ (2005) Basigin (EMMPRIN/CD147) interacts with integrin to affect cellular architecture. J Cell Sci 118:2649-2660.

Davis GW, Goodman CS (1998) Synapse-specific control of synaptic efficacy at the terminals of a single neuron. Nature 392:82-86.

Davis GW, DiAntonio A, Petersen SA, Goodman CS (1998) Postsynaptic PKA controls quantal size and reveals a retrograde signal that regulates presynaptic transmitter release in Drosophila. Neuron 20:305-315.

Del Castillo J, Stark L (1952) The effect of calcium ions on the motor end-plate potentials. J Physiol 116:507-515.

Del Castillo J, Katz B (1954) Quantal components of the end-plate potential. J Physiol 124:560-573.

Delgado R, Maureira C, Oliva C, Kidokoro Y, Labarca P (2000) Size of vesicle pools, rates of mobilization, and recycling at neuromuscular synapses of a Drosophila mutant, shibire. Neuron 28:941-953.

DiAntonio A, Petersen SA, Heckmann M, Goodman CS (1999) Glutamate receptor expression regulates quantal size and quantal content at the Drosophila neuromuscular junction. J Neurosci 19:3023-3032.

Dillon C, Goda Y (2005) The actin cytoskeleton: integrating form and function at the synapse. Annu Rev Neurosci 28:25-55.

Dingledine R, Borges K, Bowie D, Traynelis SF (1999) The glutamate receptor ion channels. Pharmacol Rev 51:7-61.

Dittman JS, Kreitzer AC, Regehr WG (2000) Interplay between facilitation, depression, and residual calcium at three presynaptic terminals. J Neurosci 20:1374-1385.

Doussau F, Augustine GJ (2000) The actin cytoskeleton and neurotransmitter release: an overview. Biochimie 82:353-363.

Dudel J (1981) The effect of reduced calcium on quantal unit current and release at the crayfish neuromuscular junction. Pflugers Arch 391:35-40.

Dutta D, Bloor JW, Ruiz-Gomez M, VijayRaghavan K, Kiehart DP (2002) Real-time imaging of morphogenetic movements in Drosophila using Gal4-UAS-driven expression of GFP fused to the actin-binding domain of moesin. Genesis 34:146-151.

Estes PS, Roos J, van der Bliek A, Kelly RB, Krishnan KS, Ramaswami M (1996) Traffic of dynamin within individual Drosophila synaptic boutons relative to compartment-specific markers. J Neurosci 16:5443-5456.

Featherstone D, Broadie K (2002) Response: meaningless minis? Trends Neurosci 25:386-387.

Featherstone DE, Davis WS, Dubreuil RR, Broadie K (2001) Drosophila alpha- and beta-spectrin mutations disrupt presynaptic neurotransmitter release. J Neurosci 21:4215-4224.

Featherstone DE, Rushton E, Rohrbough J, Liebl F, Karr J, Sheng Q, Rodesch CK, Broadie K (2005) An essential Drosophila glutamate receptor subunit that functions in both central neuropil and neuromuscular junction. J Neurosci 25:3199-3208.

Feeney CJ, Karunanithi S, Pearce J, Govind CK, Atwood HL (1998) Motor nerve terminals on abdominal muscles in larval flesh flies, Sarcophaga bullata: comparisons with Drosophila. J Comp Neurol 402:197-209. 
Feng TP (1940) Studies on the neuromuscular junction XVIII. The local potentials around n-m junctions induced by single and multiple volleys. Chin J Physiol 15:367-404.

Feng Y, Ueda A, Wu CF (2004) A modified minimal hemolymph-like solution, HL3.1, for physiological recordings at the neuromuscular junctions of normal and mutant Drosophila larvae. J Neurogenet 18:377-402.

Fenster SD, Chung WJ, Zhai R, Cases-Langhoff C, Voss B, Garner AM, Kaempf U, Kindler S, Gundelfinger ED, Garner CC (2000) Piccolo, a presynaptic zinc finger protein structurally related to bassoon. Neuron 25:203-214.

Finkel AS, Redman SJ (1985) Optimal Voltage Clamping With Single Microelectrode. In: Voltage and Patch Claming With Microelectrodes (Smith Jr. TG, Lecar H, Redman SJ, Gage PW, eds). Baltimore: Williams \& Wilkins.

Finkel AS, Gage PW (1985) Conventional Voltage Clamping Wth Two Intracellular Microelectrodes. In: Voltage and Patch Claming With Microelectrodes (Smith Jr. TG, Lecar H, Redman SJ, Gage PW, eds). Baltimore: Williams \& Wilkins.

Garner CC, Kindler S (1996) Synaptic proteins and the assembly of synaptic junctions. Trends Cell Biol 6:429-433.

Garner CC, Nash J, Huganir RL (2000a) PDZ domains in synapse assembly and signalling. Trends Cell Biol 10:274-280.

Garner CC, Kindler S, Gundelfinger ED (2000b) Molecular determinants of presynaptic active zones. Curr Opin Neurobiol 10:321-327.

Geppert M, Goda Y, Hammer RE, Li C, Rosahl TW, Stevens CF, Sudhof TC (1994) Synaptotagmin I: a major Ca2+ sensor for transmitter release at a central synapse. Cell 79:717-727.

Gramates LS, Budnik V (1999) Assembly and maturation of the Drosophila larval neuromuscular junction. Int Rev Neurobiol 43:93-117.

Hagiwara A, Fukazawa Y, Deguchi-Tawarada M, Ohtsuka T, Shigemoto R (2005) Differential distribution of release-related proteins in the hippocampal CA3 area as revealed by freeze-fracture replica labeling. J Comp Neurol 489:195-216.

Hallermann S, Pawlu C, Jonas P, Heckmann M (2003) A large pool of releasable vesicles in a cortical glutamatergic synapse. Proc Natl Acad Sci U S A 100:8975-8980.

Hamill OP, Marty A, Neher E, Sakmann B, Sigworth FJ (1981) Improved patch-clamp techniques for high-resolution current recording from cells and cell-free membrane patches. Pflugers Arch 391:85-100.

Harlow ML, Ress D, Stoschek A, Marshall RM, McMahan UJ (2001) The architecture of active zone material at the frog's neuromuscular junction. Nature 409:479484.

Hata Y, Nakanishi H, Takai Y (1998) Synaptic PDZ domain-containing proteins. Neurosci Res 32:1-7.

Hebb DO (1949) The Organization of Behavior: A Neuropsychological Theory. New York: John Wiley \& Sons.

Heckmann M, Dudel J (1995) Recordings of glutamate-gated ion channels in outsideout patches from Drosophila larval muscle. Neurosci Lett 196:53-56.

Heckmann M, Dudel J (1997) Desensitization and resensitization kinetics of glutamate receptor channels from Drosophila larval muscle. Biophys J 72:2160-2169.

Heckmann M, Dudel J (1998) Evoked quantal currents at neuromuscular junctions of wild type Drosophila larvae. Neurosci Lett 256:77-80.

Hefft S, Jonas P (2005) Asynchronous GABA release generates long-lasting inhibition at a hippocampal interneuron-principal neuron synapse. Nat Neurosci 8:13191328. 
Hell SW (2003) Toward fluorescence nanoscopy. Nat Biotechnol 21:1347-1355.

Hille B (2001) Ion Channels of Excitable Membranes, $3^{\text {rd }}$ Edition. Sunderland, Massachusetts: Sinauer Associates, Inc.

Hofbauer A (1991) Eine Bibliothek monoklonaler Antikörper gegen das Gehirn von Drosophila melanogaster. Habilitation thesis. University of Würzburg: Würzburg, Germany.

Jahn R, Lang T, Südhof TC (2003) Membrane fusion. Cell 112:519-533.

Jan LY, Jan YN (1976a) Properties of the larval neuromuscular junction in Drosophila melanogaster. J Physiol 262:189-214.

Jan LY, Jan YN (1976b) L-glutamate as an excitatory transmitter at the Drosophila larval neuromuscular junction. J Physiol 262:215-236.

Jia XX, Gorczyca M, Budnik V (1993) Ultrastructure of neuromuscular junctions in Drosophila: comparison of wild type and mutants with increased excitability. J Neurobiol 24:1025-1044.

Jonas P, Spruston N (1994) Mechanisms shaping glutamate-mediated excitatory postsynaptic currents in the CNS. Curr Opin Neurobiol 4:366-372.

Kandel ER (1976) Cellular Basis of Behaviour: An introduction to Behavioural Neurobiology. San Francisco: W.H. Freeman.

Kandel ER (2001) The molecular biology of memory storage: a dialogue between genes and synapses. Science 294:1030-1038.

Katz B (1969) The release of neural transmitter substances. Liverpool, UK: Liverpool UP.

Katz B, Miledi R (1965) The Effect Of Calcium On Acetylcholine Release From Motor Nerve Terminals. Proc R Soc Lond B Biol Sci 161:496-503.

Katz B, Miledi R (1968) The role of calcium in neuromuscular facilitation. J Physiol 195:481-492.

Kawasaki F, Felling R, Ordway RW (2000) A temperature-sensitive paralytic mutant defines a primary synaptic calcium channel in Drosophila. J Neurosci 20:48854889.

Kawasaki F, Zou B, Xu X, Ordway RW (2004) Active zone localization of presynaptic calcium channels encoded by the cacophony locus of Drosophila. J Neurosci 24:282-285.

Kennedy MB (1997) The postsynaptic density at glutamatergic synapses. Trends Neurosci 20:264-268.

Keshishian H, Chiba A, Chang TN, Halfon MS, Harkins EW, Jarecki J, Wang L, Anderson M, Cash S, Halpern ME, et al. (1993) Cellular mechanisms governing synaptic development in Drosophila melanogaster. J Neurobiol 24:757-787.

Khimich D, Nouvian R, Pujol R, Tom Dieck S, Egner A, Gundelfinger ED, Moser T (2005) Hair cell synaptic ribbons are essential for synchronous auditory signalling. Nature 434:889-894.

Kidokoro Y, Nishikawa K (1994) Miniature endplate currents at the newly formed neuromuscular junction in Drosophila embryos and larvae. Neurosci Res 19:143-154.

Kittel RJ, Hallermann S, Thomsen S, Wichmann C, Sigrist SJ, Heckmann M (2006a) Active zone assembly and synaptic release. Biochem Soc Trans 34:944-946.

Kittel RJ, Wichmann C, Rasse TM, Fouquet W, Schmidt M, Schmid A, Wagh DA, Pawlu C, Kellner RR, Willig KI, Hell SW, Buchner E, Heckmann M, Sigrist SJ (2006b) Bruchpilot promotes active zone assembly, Ca2+ channel clustering, and vesicle release. Science 312:1051-1054.

Klagges BR, Heimbeck G, Godenschwege TA, Hofbauer A, Pflugfelder GO, Reifegerste R, Reisch D, Schaupp M, Buchner S, Buchner E (1996) Invertebrate 
synapsins: a single gene codes for several isoforms in Drosophila. J Neurosci 16:3154-3165.

Klar TA, Jakobs S, Dyba M, Egner A, Hell SW (2000) Fluorescence microscopy with diffraction resolution barrier broken by stimulated emission. Proc Natl Acad Sci U S A 97:8206-8210.

Klingauf J, Kavalali ET, Tsien RW (1998) Kinetics and regulation of fast endocytosis at hippocampal synapses. Nature 394:581-585.

Koenig JH, Ikeda K (1996) Synaptic vesicles have two distinct recycling pathways. J Cell Biol 135:797-808.

Koester HJ, Johnston D (2005) Target cell-dependent normalization of transmitter release at neocortical synapses. Science 308:863-866.

Koh TW, Bellen HJ (2003) Synaptotagmin I, a Ca2+ sensor for neurotransmitter release. Trends Neurosci 26:413-422.

Koh TW, Verstreken P, Bellen HJ (2004) Dap160/intersectin acts as a stabilizing scaffold required for synaptic development and vesicle endocytosis. Neuron 43:193-205.

Konorski J (1948) Conditional Reflexes and Neuron Organization. Cambridge: Cambridge Univ. Press.

Kuffler SW (1942) The effect of calcium on the neuromuscular junction. J Neurophysiol 7:17-26.

Kurdyak P, Atwood HL, Stewart BA, Wu CF (1994) Differential physiology and morphology of motor axons to ventral longitudinal muscles in larval Drosophila. J Comp Neurol 350:463-472.

Kuromi H, Kidokoro Y (1998) Two distinct pools of synaptic vesicles in single presynaptic boutons in a temperature-sensitive Drosophila mutant, shibire. Neuron 20:917-925.

Kuromi H, Kidokoro Y (2003) Two synaptic vesicle pools, vesicle recruitment and replenishment of pools at the Drosophila neuromuscular junction. J Neurocytol 32:551-565.

Kuromi H, Honda A, Kidokoro Y (2004) $\mathrm{Ca}^{2+}$ Influx through Distinct Routes Controls Exocytosis and Endocytosis at Drosophila Presynaptic Terminals. Neuron 41:101-111.

Laissue PP, Reiter C, Hiesinger PR, Halter S, Fischbach KF, Stocker RF (1999) Threedimensional reconstruction of the antennal lobe in Drosophila melanogaster. $\mathrm{J}$ Comp Neurol 405:543-552.

Landis DM, Hall AK, Weinstein LA, Reese TS (1988) The organization of cytoplasm at the presynaptic active zone of a central nervous system synapse. Neuron 1:201209.

Lenzi D, von Gersdorff H (2001) Structure suggests function: the case for synaptic ribbons as exocytotic nanomachines. Bioessays 23:831-840.

Linden DJ, Connor JA (1995) Long-term synaptic depression. Annu Rev Neurosci 18:319-357.

Llinas R, Steinberg IZ, Walton K (1981) Relationship between presynaptic calcium current and postsynaptic potential in squid giant synapse. Biophys J 33:323-351.

Locke FS (1894) Notiz über den Einfluss physiologischer Kochsalzlösung auf die elektrische Erregbarkeit von Muskel und Nerv. zbl Physiol 8:166-167.

Loewi O (1921) Über humorale Übertragbarkeit der Herznervenwirkung. Pflügers Arch 189:239-242.

Lomeli H, Mosbacher J, Melcher T, Hoger T, Geiger JR, Kuner T, Monyer H, Higuchi M, Bach A, Seeburg PH (1994) Control of kinetic properties of AMPA receptor channels by nuclear RNA editing. Science 266:1709-1713. 
Madden DR (2002) The structure and function of glutamate receptor ion channels. Nat Rev Neurosci 3:91-101.

Magleby KL (1987) Short-term changes in synaptic efficacy. In: Synaptic Function (Edelman GM, Gall WE, Cowan WM, eds), pp 21-56. New York: John Wiley \& Sons.

Malinow R, Malenka RC (2002) AMPA receptor trafficking and synaptic plasticity. Annu Rev Neurosci 25:103-126.

Marie B, Sweeney ST, Poskanzer KE, Roos J, Kelly RB, Davis GW (2004) Dap160/intersectin scaffolds the periactive zone to achieve high-fidelity endocytosis and normal synaptic growth. Neuron 43:207-219.

Marrus SB, Portman SL, Allen MJ, Moffat KG, DiAntonio A (2004) Differential localization of glutamate receptor subunits at the Drosophila neuromuscular junction. J Neurosci 24:1406-1415.

Martin KC, Barad M, Kandel ER (2000) Local protein synthesis and its role in synapsespecific plasticity. Curr Opin Neurobiol 10:587-592.

Menon KP, Sanyal S, Habara Y, Sanchez R, Wharton RP, Ramaswami M, Zinn K (2004) The translational repressor Pumilio regulates presynaptic morphology and controls postsynaptic accumulation of translation factor eIF-4E. Neuron 44:663-676.

Morales M, Colicos MA, Goda Y (2000) Actin-dependent regulation of neurotransmitter release at central synapses. Neuron 27:539-550.

Mosbacher J, Schoepfer R, Monyer H, Burnashev N, Seeburg PH, Ruppersberg JP (1994) A molecular determinant for submillisecond desensitization in glutamate receptors. Science 266:1059-1062.

Neher E (1981) Unit conductance studies in biological membranes. In: Techniques in cellular physiology (Baker PF, ed). Amsterdam, Holland: Elsevier/North.

Neher E (1998) Vesicle pools and $\mathrm{Ca}^{2+}$ microdomains: new tools for understanding their roles in neurotransmitter release. Neuron 20:389-399.

Neher E, Sakaba T (2001) Combining deconvolution and noise analysis for the estimation of transmitter release rates at the calyx of held. J Neurosci 21:444461.

Neher E, Sakaba T (2003) Combining deconvolution and fluctuation analysis to determine quantal parameters and release rates. J Neurosci Methods 130:143157.

Nicoll RA, Tomita S, Bredt DS (2006) Auxiliary subunits assist AMPA-type glutamate receptors. Science 311:1253-1256.

Nishikawa K, Kidokoro Y (1995) Junctional and extrajunctional glutamate receptor channels in Drosophila embryos and larvae. J Neurosci 15:7905-7915.

Ohtsuka T, Takao-Rikitsu E, Inoue E, Inoue M, Takeuchi M, Matsubara K, DeguchiTawarada M, Satoh K, Morimoto K, Nakanishi H, Takai Y (2002) Cast: a novel protein of the cytomatrix at the active zone of synapses that forms a ternary complex with RIM1 and munc13-1. J Cell Biol 158:577-590.

Packard M, Mathew D, Budnik V (2003) FASt remodeling of synapses in Drosophila. Curr Opin Neurobiol 13:527-534.

Paradis S, Sweeney ST, Davis GW (2001) Homeostatic control of presynaptic release is triggered by postsynaptic membrane depolarization. Neuron 30:737-749.

Pawlu C, DiAntonio A, Heckmann M (2004) Postfusional control of quantal current shape. Neuron 42:607-618.

Peters A, Palay SL, Webster HD (1991) The fine structure of the nervous system, $3^{\text {rd }}$ Edition. New York, Oxford: Oxford University Press. 
Petersen SA, Fetter RD, Noordermeer JN, Goodman CS, DiAntonio A (1997) Genetic analysis of glutamate receptors in Drosophila reveals a retrograde signal regulating presynaptic transmitter release. Neuron 19:1237-1248.

Phillips GR, Huang JK, Wang Y, Tanaka H, Shapiro L, Zhang W, Shan WS, Arndt K, Frank M, Gordon RE, Gawinowicz MA, Zhao Y, Colman DR (2001) The presynaptic particle web: ultrastructure, composition, dissolution, and reconstitution. Neuron 32:63-77.

Pinheiro P, Mulle C (2006) Kainate receptors. Cell Tissue Res.

Polo-Parada L, Bose CM, Landmesser LT (2001) Alterations in transmission, vesicle dynamics, and transmitter release machinery at NCAM-deficient neuromuscular junctions. Neuron 32:815-828.

Prokop A (1999) Integrating bits and pieces: synapse structure and formation in Drosophila embryos. Cell Tissue Res 297:169-186.

Prokop A, Meinertzhagen IA (2006) Development and structure of synaptic contacts in Drosophila. Semin Cell Dev Biol 17:20-30.

Qin G, Schwarz T, Kittel RJ, Schmid A, Rasse TM, Kappei D, Ponimaskin E, Heckmann M, Sigrist SJ (2005) Four different subunits are essential for expressing the synaptic glutamate receptor at neuromuscular junctions of Drosophila. J Neurosci 25:3209-3218.

Ramón y Cajal S (1894) The Croonian Lecture: La fine structure des centres nerveux. Proc R Soc London B 55:444-467.

Rasse TM, Fouquet W, Schmid A, Kittel RJ, Mertel S, Sigrist CB, Schmidt M, Guzman A, Merino C, Qin G, Quentin C, Madeo FF, Heckmann M, Sigrist SJ (2005) Glutamate receptor dynamics organizing synapse formation in vivo. Nat Neurosci 8:898-905.

Renger JJ, Ueda A, Atwood HL, Govind CK, Wu CF (2000) Role of cAMP cascade in synaptic stability and plasticity: ultrastructural and physiological analyses of individual synaptic boutons in Drosophila memory mutants. J Neurosci 20:39803992.

Richards DA, Rizzoli SO, Betz WJ (2004) Effects of wortmannin and latrunculin A on slow endocytosis at the frog neuromuscular junction. J Physiol 557:77-91.

Rizzoli SO, Betz WJ (2005) Synaptic vesicle pools. Nat Rev Neurosci 6:57-69.

Rodesch CK, Broadie K (2000) Genetic studies in Drosophila: vesicle pools and cytoskeleton-based regulation of synaptic transmission. Neuroreport 11:R45-53.

Rosenmund C, Stern-Bach Y, Stevens CF (1998) The tetrameric structure of a glutamate receptor channel. Science 280:1596-1599.

Rosenmund C, Rettig J, Brose N (2003) Molecular mechanisms of active zone function. Curr Opin Neurobiol 13:509-519.

Rougon G, Hobert O (2003) New insights into the diversity and function of neuronal immunoglobulin superfamily molecules. Annu Rev Neurosci 26:207-238.

Rozov A, Burnashev N, Sakmann B, Neher E (2001) Transmitter release modulation by intracellular $\mathrm{Ca}^{2+}$ buffers in facilitating and depressing nerve terminals of pyramidal cells in layer $2 / 3$ of the rat neocortex indicates a target cell-specific difference in presynaptic calcium dynamics. J Physiol 531:807-826.

Ruiz-Canada C, Ashley J, Moeckel-Cole S, Drier E, Yin J, Budnik V (2004) New synaptic bouton formation is disrupted by misregulation of microtubule stability in aPKC mutants. Neuron 42:567-580.

Sakaba T (2006) Roles of the fast-releasing and the slowly releasing vesicles in synaptic transmission at the calyx of held. J Neurosci 26:5863-5871.

Sakaba T, Neher E (2001) Calmodulin mediates rapid recruitment of fast-releasing synaptic vesicles at a calyx-type synapse. Neuron 32:1119-1131. 
Sakaba T, Neher E (2003) Involvement of actin polymerization in vesicle recruitment at the calyx of Held synapse. J Neurosci 23:837-846.

Sakaba T, Stein A, Jahn R, Neher E (2005) Distinct kinetic changes in neurotransmitter release after SNARE protein cleavage. Science 309:491-494.

Sankaranarayanan S, Atluri PP, Ryan TA (2003) Actin has a molecular scaffolding, not propulsive, role in presynaptic function. Nat Neurosci 6:127-135.

Sara Y, Virmani T, Deak F, Liu X, Kavalali ET (2005) An isolated pool of vesicles recycles at rest and drives spontaneous neurotransmission. Neuron 45:563-573.

Schmid A, Qin G, Wichmann C, Kittel RJ, Mertel S, Fouquet W, Schmidt M, Heckmann M, Sigrist SJ (2006) Non NMDA type glutamate receptors are essential for maturation but not for initial assembly of synapses at Drosophila NMJs. J Neurosci 26:11267-11277.

Schneggenburger R, Neher E (2005) Presynaptic calcium and control of vesicle fusion. Curr Opin Neurobiol 15:266-274.

Schuster CM, Davis GW, Fetter RD, Goodman CS (1996a) Genetic dissection of structural and functional components of synaptic plasticity. I. Fasciclin II controls synaptic stabilization and growth. Neuron 17:641-654.

Schuster CM, Davis GW, Fetter RD, Goodman CS (1996b) Genetic dissection of structural and functional components of synaptic plasticity. II. Fasciclin II controls presynaptic structural plasticity. Neuron 17:655-667.

Schuster CM, Ultsch A, Schloss P, Cox JA, Schmitt B, Betz H (1991) Molecular cloning of an invertebrate glutamate receptor subunit expressed in Drosophila muscle. Science 254:112-114.

Seeburg PH (1993) The TiPS/TINS lecture: the molecular biology of mammalian glutamate receptor channels. Trends Pharmacol Sci 14:297-303.

Sheng M, Pak DT (1999) Glutamate receptor anchoring proteins and the molecular organization of excitatory synapses. Ann N Y Acad Sci 868:483-493.

Sheng M, Sala C (2001) PDZ domains and the organization of supramolecular complexes. Annu Rev Neurosci 24:1-29.

Shi S, Hayashi Y, Esteban JA, Malinow R (2001) Subunit-specific rules governing AMPA receptor trafficking to synapses in hippocampal pyramidal neurons. Cell 105:331-343.

Shupliakov O, Bloom O, Gustafsson JS, Kjaerulff O, Low P, Tomilin N, Pieribone VA, Greengard P, Brodin L (2002) Impaired recycling of synaptic vesicles after acute perturbation of the presynaptic actin cytoskeleton. Proc Natl Acad Sci U S A 99:14476-14481.

Sigrist SJ, Thiel PR, Reiff DF, Schuster CM (2002) The postsynaptic glutamate receptor subunit DGluR-IIA mediates long-term plasticity in Drosophila. J Neurosci 22:7362-7372.

Sigrist SJ, Reiff DF, Thiel PR, Steinert JR, Schuster CM (2003) Experience-dependent strengthening of Drosophila neuromuscular junctions. J Neurosci 23:6546-6556.

Sigrist SJ, Thiel PR, Reiff DF, Lachance PE, Lasko P, Schuster CM (2000) Postsynaptic translation affects the efficacy and morphology of neuromuscular junctions. Nature 405:1062-1065.

Sigworth FJ, Neher E (1980) Single Na+ channel currents observed in cultured rat muscle cells. Nature 287:447-449.

Singh S, Wu CF (1999) Ionic currents in larval muscles of Drosophila. Int Rev Neurobiol 43:191-220.

Slayter HS, Lowey S (1967) Substructure of the myosin molecule as visualized by electron microscopy. Proc Natl Acad Sci U S A 58:1611-1618. 
Smart TG (1997) Regulation of excitatory and inhibitory neurotransmitter-gated ion channels by protein phosphorylation. Curr Opin Neurobiol 7:358-367.

Sone M, Suzuki E, Hoshino M, Hou D, Kuromi H, Fukata M, Kuroda S, Kaibuchi K, Nabeshima Y, Hama C (2000) Synaptic development is controlled in the periactive zones of Drosophila synapses. Development 127:4157-4168.

Steinert JR, Kuromi H, Hellwig A, Knirr M, Wyatt AW, Kidokoro Y, Schuster CM (2006) Experience-dependent formation and recruitment of large vesicles from reserve pool. Neuron 50:723-733.

Steward O, Schuman EM (2001) Protein synthesis at synaptic sites on dendrites. Annu Rev Neurosci 24:299-325.

Stewart BA, Schuster CM, Goodman CS, Atwood HL (1996) Homeostasis of synaptic transmission in Drosophila with genetically altered nerve terminal morphology. J Neurosci 16:3877-3886.

Stewart BA, Atwood HL, Renger JJ, Wang J, Wu CF (1994) Improved stability of Drosophila larval neuromuscular preparations in haemolymph-like physiological solutions. J Comp Physiol [A] 175:179-191.

Südhof TC (2004) The synaptic vesicle cycle. Annu Rev Neurosci 27:509-547.

Takao-Rikitsu E, Mochida S, Inoue E, Deguchi-Tawarada M, Inoue M, Ohtsuka T, Takai Y (2004) Physical and functional interaction of the active zone proteins, CAST, RIM1, and Bassoon, in neurotransmitter release. J Cell Biol 164:301311.

TheAxonGuide (1993) The Axon Guide for Electrophysiology \& Biophysics Laboratory Techniques. Foster City, CA: Axon Instruments, Inc.

TheFlyBaseConsortium (2003) The FlyBase database of the Drosophila genome projects and community literature. Nucleic Acids Res 31:172-175.

tom Dieck S, Altrock WD, Kessels MM, Qualmann B, Regus H, Brauner D, Fejtova A, Bracko O, Gundelfinger ED, Brandstatter JH (2005) Molecular dissection of the photoreceptor ribbon synapse: physical interaction of Bassoon and RIBEYE is essential for the assembly of the ribbon complex. J Cell Biol 168:825-836.

tom Dieck S, Sanmarti-Vila L, Langnaese K, Richter K, Kindler S, Soyke A, Wex H, Smalla KH, Kampf U, Franzer JT, Stumm M, Garner CC, Gundelfinger ED (1998) Bassoon, a novel zinc-finger CAG/glutamine-repeat protein selectively localized at the active zone of presynaptic nerve terminals. J Cell Biol 142:499509.

Trommershäuser J, Schneggenburger R, Zippelius A, Neher E (2003) Heterogeneous presynaptic release probabilities: functional relevance for short-term plasticity. Biophys J 84:1563-1579.

Trujillo-Cenoz O (1969) Some aspects of the structural organization of the medulla in muscoid flies. J Ultrastruct Res 27:533-553.

Turrigiano GG, Leslie KR, Desai NS, Rutherford LC, Nelson SB (1998) Activitydependent scaling of quantal amplitude in neocortical neurons. Nature 391:892896.

von Gersdorff H (2001) Synaptic ribbons: versatile signal transducers. Neuron 29:7-10. von Gersdorff H, Schneggenburger R, Weis S, Neher E (1997) Presynaptic depression at a calyx synapse: the small contribution of metabotropic glutamate receptors. J Neurosci 17:8137-8146.

Wagh DA, Rasse TM, Asan E, Hofbauer A, Schwenkert I, Dürrbeck H, Buchner S, Dabauvalle MC, Schmidt M, Qin G, Wichmann C, Kittel R, Sigrist SJ, Buchner E (2006) Bruchpilot, a Protein with Homology to ELKS/CAST, Is Required for Structural Integrity and Function of Synaptic Active Zones in Drosophila. Neuron 49:833-844. 
Wang XH, Zheng JQ, Poo MM (1996) Effects of cytochalasin treatment on short-term synaptic plasticity at developing neuromuscular junctions in frogs. J Physiol 491 (Pt 1):187-195.

Wang Y, Sugita S, Sudhof TC (2000) The RIM/NIM family of neuronal C2 domain proteins. Interactions with Rab3 and a new class of Src homology 3 domain proteins. J Biol Chem 275:20033-20044.

Wang Y, Liu X, Biederer T, Sudhof TC (2002) A family of RIM-binding proteins regulated by alternative splicing: Implications for the genesis of synaptic active zones. Proc Natl Acad Sci U S A 99:14464-14469.

Weigmann K, Klapper R, Strasser T, Rickert C, Technau G, Jackle H, Janning W, Klambt C (2003) FlyMove--a new way to look at development of Drosophila. Trends Genet 19:310-311.

Wells DG, Richter JD, Fallon JR (2000) Molecular mechanisms for activity-regulated protein synthesis in the synapto-dendritic compartment. Curr Opin Neurobiol 10:132-137.

Wojtowicz JM, Marin L, Atwood HL (1994) Activity-induced changes in synaptic release sites at the crayfish neuromuscular junction. J Neurosci 14:3688-3703.

Wu CF, Haugland FN (1985) Voltage clamp analysis of membrane currents in larval muscle fibers of Drosophila: alteration of potassium currents in Shaker mutants. J Neurosci 5:2626-2640.

Wu L, Wells D, Tay J, Mendis D, Abbott MA, Barnitt A, Quinlan E, Heynen A, Fallon JR, Richter JD (1998) CPEB-mediated cytoplasmic polyadenylation and the regulation of experience-dependent translation of alpha-CaMKII mRNA at synapses. Neuron 21:1129-1139.

$\mathrm{Wu} \mathrm{W}, \mathrm{Xu} \mathrm{J}, \mathrm{Wu} \mathrm{XS}, \mathrm{Wu}$ LG (2005) Activity-dependent acceleration of endocytosis at a central synapse. J Neurosci 25:11676-11683.

Wucherpfennig T, Wilsch-Brauninger M, Gonzalez-Gaitan M (2003) Role of Drosophila Rab5 during endosomal trafficking at the synapse and evoked neurotransmitter release. J Cell Biol 161:609-624.

Yamagata M, Sanes JR, Weiner JA (2003) Synaptic adhesion molecules. Curr Opin Cell Biol 15:621-632.

Yoshihara M, Adolfsen B, Galle KT, Littleton JT (2005) Retrograde signaling by Syt 4 induces presynaptic release and synapse-specific growth. Science 310:858-863.

Zenisek D, Steyer JA, Almers W (2000) Transport, capture and exocytosis of single synaptic vesicles at active zones. Nature 406:849-854.

Zhai RG, Bellen HJ (2004) The architecture of the active zone in the presynaptic nerve terminal. Physiology (Bethesda) 19:262-270.

Zhang YQ, Rodesch CK, Broadie K (2002) Living synaptic vesicle marker: synaptotagmin-GFP. Genesis 34:142-145.

Zhong Y, Budnik V, Wu CF (1992) Synaptic plasticity in Drosophila memory and hyperexcitable mutants: role of cAMP cascade. J Neurosci 12:644-651.

Zhu JJ, Esteban JA, Hayashi Y, Malinow R (2000) Postnatal synaptic potentiation: delivery of GluR4-containing AMPA receptors by spontaneous activity. Nat Neurosci 3:1098-1106.

Zucker RS, Regehr WG (2002) Short-term synaptic plasticity. Annu Rev Physiol 64:355-405. 


\section{Appendix}

\subsection{Publications}

Besse F, Mertel S, Kittel RJ, Wichmann C, Rasse TM, Sigrist SJ, Ephrussi A (2006) The Ig CAM Basigin controls the distribution and release of synaptic vesicles at Drosophila neuromuscular junctions. Submitted.

Kittel RJ, Hallermann S, Thomsen S, Wichmann C, Sigrist SJ, Heckmann M (2006) Active zone assembly and synaptic release. Biochem Soc Trans 34: 944-946.

Kittel RJ, Wichmann C, Rasse TM, Fouquet W, Schmidt M, Schmid A, Wagh DA, Pawlu C, Kellner RR, Willig KI, Hell SW, Buchner E, Heckmann M, Sigrist SJ (2006) Bruchpilot promotes active zone assembly, $\mathrm{Ca}^{2+}$ clustering, and vesicle release. Science 312: 1051-1054.

Qin G, Schwarz T, Kittel RJ, Schmid A, Rasse TM, Kappei D, Ponimaskin E, Heckmann M, Sigrist SJ (2005) Four different subunits are essential for expressing the synaptic glutamate receptor at neuromuscular junctions of Drosophila. J Neurosci 25: 3209-3218.

Rasse TM, Fouquet W, Schmid A, Kittel RJ, Mertel S, Sigrist CB, Schmidt M, Guzman A, Merino C, Qin G, Quentin C, Madeo FF, Heckmann M, Sigrist SJ (2005) Glutamate receptor dynamics organizing synapse formation in vivo. Nat Neurosci 8: 898-905.

Schmid A, Qin G, Wichmann C, Kittel RJ, Mertel S, Fouquet W, Schmidt M, Heckmann M, Sigrist SJ (2006) Non NMDA type glutamate receptors are essential for maturation but not for initial assembly of synapses at Drosophila NMJs. J Neurosci 26: 11267-11277.

Wagh DA, Rasse TM, Asan E, Hofbauer A, Schwenkert I, Dürrbeck H, Buchner S, Dabauvalle MC, Schmidt M, Qin G, Wichmann C, Kittel R, Sigrist SJ, Buchner E (2006) Bruchpilot, a Protein with Homology to ELKS/CAST, Is Required for Structural Integrity and Function of Synaptic Active Zones in Drosophila. Neuron 49: 833-844. 


\subsection{Curriculum vitae}

\section{Personal details}

Surname:

Kittel

Forenames:

Robert Johannes

Nationality:

German and British

Date and place of birth:

Marital status:

Work address:

11.01.1978 in Berlin, Germany

Single

ENI

Grisebachstr. 5

37077 Göttingen, Germany

Tel.: (+49)-(0)551-3912357

e-mail: rkittel@gwdg.de

\section{Scientific education}

1990-1997

1998-2002

2002

2003-present

Scientific work

August-September 2001

2002

September-October 2004
Felix- Klein Gymnasium, Göttingen, Germany

Abitur (university entrance qualification)

Undergraduate studies at the University of Glasgow, Scotland, UK

Bachelor of Science (B.Sc.) with Honours in Neurosciences

$\mathrm{PhD}$ (Doctor rerum naturalium) student in the Neuroplasticity group, ENI, Göttingen, Germany
Internship "Characterisation of Complexins III and IV" Abt. Molekulare Neurobiologie, Prof. Dr. Nils Brose Max-Planck-Institut für Experimentelle Medizin, Göttingen, Germany

Supervisor: Dr. Kerstin Reim

Undergraduate thesis: "The involvement of Erk1 and Dynamin1 in the early phase of long-term potentiation". Institute of Biomedical and Life Sciences University of Glasgow, Scotland, UK Supervisor: Dr. Stuart Cobb

Internship "Patch-clamp techniques" Physiologisches Institut, Prof. Dr. Peter Jonas Albert-Ludwigs-Universität Freiburg, Germany Supervisor: Prof. Dr. Manfred Heckmann 
$\mathrm{PhD}$ (Doctor rerum naturalium) thesis: "Synaptic physiology of the developing Drosophila neuromuscular junction".

Georg-August Universität Göttingen, Germany

Referee: Prof. Dr. Erwin Neher

Co-referee: Prof. Dr. Friedrich-Wilhelm Schürmann

Instructor: Prof. Dr. Stephan Sigrist

\section{Selected scientific presentations}

Schmid A, Kittel RJ, Qin G, Rasse TM, Guzman A, Fouquet W, Sigrist SJ (2005) Subunit-specific glutamate receptor dynamics during synapse formation and remodelling in Drosophila. Gordon research conference on Excitatory Amino Acids \& Brain Function, Aussois, France.

Kittel RJ, Wichmann C, Rasse TM, Fouquet W, Schmidt M, Schmid A, Wagh DA, Pawlu C, Kellner RR, Willig KI, Hell SW, Buchner E, Heckmann M, Sigrist SJ (2006) Drosophila Bruchpilot promotes presynaptic active zone assembly and $\mathrm{Ca}^{2+}$ clustering to ensure efficient vesicle release. Joint meeting of the German Physiological Society and the European Federation of Physiological Societies, München, Germany.

Kittel RJ, Wichmann C, Rasse TM, Fouquet W, Schmidt M, Schmid A, Wagh DA, Pawlu C, Kellner RR, Willig KI, Hell SW, Buchner E, Heckmann M, Sigrist SJ (2006) Drosophila Bruchpilot is required for presynaptic active zone assembly and $\mathrm{Ca}^{2+}$ clustering to ensure efficient vesicle release. Bioscience 2006, incorporating the Biochemical Journal Centenary Symposium, Glasgow, Scotland, UK. 